\title{
THE SOLAR NEIGHBORHOOD. XXXVIII. RESULTS FROM THE CTIO/SMARTS 0.9 m: TRIGONOMETRIC PARALLAXES FOR 151 NEARBY M DWARF SYSTEMS
}

\author{
Jennifer G. Winters ${ }^{1,8}$, R. Andrew Sevrinsky ${ }^{2,8}$, Wei-Chun JaO ${ }^{2,8}$, Todd J. Henry ${ }^{3,8}$, Adric R. Riedel ${ }^{4,8}$, \\ John P. Subasavage ${ }^{5,8}$, John C. Lurie ${ }^{6,8}$, Philip A. Ianna ${ }^{3,8}$, and Charlie T. Finch ${ }^{7,8}$ \\ ${ }^{1}$ Harvard-Smithsonian Center for Astrophysics, Cambridge, MA 02138, USA; jennifer.winters@cfa.harvard.edu \\ ${ }^{2}$ Department of Physics and Astronomy, Georgia State University, Atlanta, GA 30302-4106, USA; sevrinsky@astro.gsu.edu, jao@astro.gsu.edu \\ ${ }^{3}$ RECONS Institute, Chambersburg, PA 17201, USA; toddhenry28@gmail.com, philianna3@gmail.com \\ ${ }^{4}$ Astronomy Department, California Institute of Technology, Pasadena, CA 91125, USA; arr@astro.caltech.edu \\ ${ }^{5}$ US Naval Observatory, Flagstaff Station, 10391 West Observatory Road, Flagstaff, AZ 86001, USA; jsubasavage@ @nofs.navy.mil \\ ${ }^{6}$ Astronomy Department, University of Washington, Seattle, WA 98195, USA; lurie@uw.edu \\ ${ }^{7}$ US Naval Observatory, 3450 Massachussetts Ave. NW, Washington, DC 20392, USA; finch@usno.navy.mil \\ Received 2016 August 5; revised 2016 October 18; accepted 2016 October 24; published 2016 December 20
}

\begin{abstract}
We present 160 new trigonometric parallaxes for $151 \mathrm{M}$ dwarf systems from the REsearch Consortium On Nearby Stars (RECONS) group's long-term astrometry/photometry program at the CTIO/SMARTS $0.9 \mathrm{~m}$

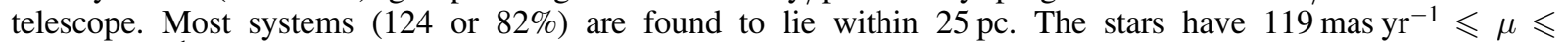
$828 \mathrm{mas} \mathrm{yr}^{-1}$ and $3.85 \leqslant(V-K) \leqslant 8.47$. Among these are 58 systems from the SuperCOSMOS-RECONS search, discovered via our proper motion trawls of the SuperCOSMOS digitized archival photographic plates, while the remaining stars were suspected via photometric distance estimates to lie nearby. Sixteen systems were newly discovered via astrometric perturbations to be binaries, many of which are ideal for accurate mass determinations due to their proximity and orbital periods on the order of a decade. A variability analysis of the stars presented, two-thirds of which are new results, shows six of the stars to vary by more than 20 mmag. This effort brings the total number of parallaxes for $\mathrm{M}$ dwarf systems measured by RECONS to nearly 500 and increases by $26 \%$ the number of southern M dwarf systems with accurate trigonometric parallaxes placing them within $25 \mathrm{pc}$.
\end{abstract}

Key words: parallaxes - solar neighborhood - stars: distances - stars: low-mass - stars: statistics

\section{INTRODUCTION}

Historically, trigonometric parallax $\left(\pi_{\text {trig }}\right)$ measurements for $\mathrm{M}$ dwarfs have been a challenge due to the intrinsic faintness of these low-mass stars. Thus, early astrometric programs typically targeted the brightest and earliest of $\mathrm{M}$ dwarf spectral types, which were usually also the nearest. The first publication of parallaxes for $\mathrm{M}$ dwarf primaries reported results for stars with $V<10$ from the Yerkes $1.0 \mathrm{~m}$ (Schlesinger 1911). Today, the two cornerstones of parallax results are The General Catalogue of Trigonometric Parallaxes, Fourth Edition (van Altena et al. 1995) from Yale University Observatory, often called the Yale Parallax Catalog (hereafter referred to as YPC) and the HIPPARCOS catalog (Perryman et al. 1997, updated in van Leeuwen 2007; hereafter referred to as HIP), with many of the brighter YPC M dwarfs also having been observed by HIP. Most of the parallaxes for nearby M dwarfs in the YPC are results from the long-term astrometric programs that were carried out with telescopes at the Allegheny, Cape, McCormick, Mt. Wilson, Sproul, Van Vleck, Yale, Yerkes, and US Naval Observatories. The $\sim 475$ nearby $M$ dwarf systems included in HIP are bright $(V \lesssim 12)$ and mostly early-type objects, while the $\sim 650$ nearby $\mathrm{M}$ dwarfs listed in the YPC encompass a wide range of $\mathrm{M}$ dwarf spectral types. The faintest M dwarf included in YPC (RGO 0050-2722, with $V=21.51$; Ianna 1993) is due to astrometrists' continuing efforts to push the faintness limits of parallax measurements (Ianna \& Bessell 1986).

\footnotetext{
${ }^{8}$ Visiting Astronomer, Cerro Tololo Inter-American Observatory. CTIO is operated by AURA, Inc. under contract to the National Science Foundation.
}

Recent interest in M dwarfs as targets for exoplanet searches has spurred parallax measurements for these stars at the bottom of the stellar main-sequence. Many astrometric programs (Smart et al. 2007, 2010; Gatewood 2008; Gatewood \& Coban 2009; Lèpine et al. 2009; Dupuy \& Liu 2012; Shkolnik et al. 2012; Dittmann et al. 2014; Finch \& Zacharias 2016) have contributed important distance measurements to the nearby $\mathrm{M}$ dwarf population, primarily in the northern hemisphere. Additional results have become available from rereductions of the Hipparcos data, e.g., Söderhjelm (1999) and Fabricius \& Makarov (2000).

The REsearch Consortium On Nearby Stars' (RECONS) ${ }^{9}$ work over the past two decades has helped fill in the nearby star census with vital distance determinations, specifically in the historically neglected southern hemisphere. To date, RECONS has contributed the first reliable $\pi_{\text {trig }}$ measurements for $\sim 200 \mathrm{M}$ dwarfs, as well as for white dwarfs and brown dwarfs, within $25 \mathrm{pc}$ via our long-term astrometric program at the Cerro Tololo Inter-American Observatory/Small and Moderate Aperture Research Telescope System (CTIO/SMARTS) $0.9 \mathrm{~m}$ and $1.5 \mathrm{~m}$ (Henry et al. 1997, 2006; Costa et al. 2005, 2006; Jao et al. 2005, 2011, 2014; Gizis et al. 2007; Subasavage et al. 2009; Riedel et al. 2010, 2011, 2014; von Braun et al. 2011; Mamajek et al. 2013; Dieterich et al. 2014; Lurie et al. 2014; Davison et al. 2015). The work presented here is part of the continuing RECONS effort to determine a complete census of all $\mathrm{M}$ dwarfs within $25 \mathrm{pc}$. Here we present the largest set of accurate $\pi_{\text {trig }}$ measurements for stars in the southern hemisphere since the YPC and HIP.

\footnotetext{
www.recons.org
} 

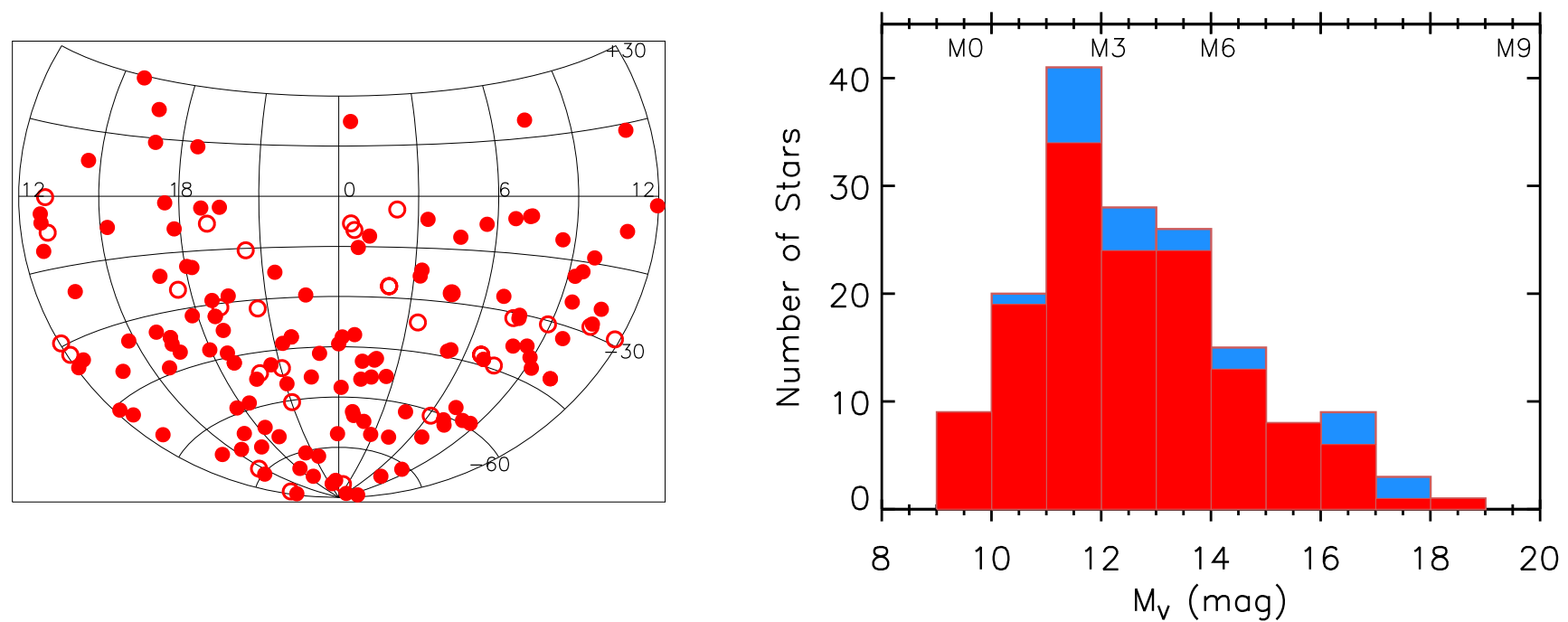

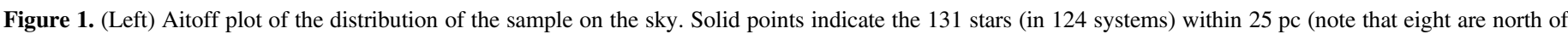

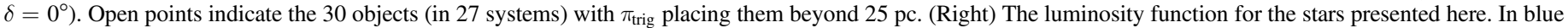

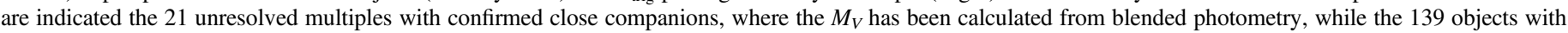
individual photometry are shown in red. Spectral type estimates are indicated along the top of the plot.

\section{DEFINITION OF THE SAMPLE}

Stars reported here were targeted during the astrometry program because they were likely to be red dwarfs within $25 \mathrm{pc}$ with no previous published $\pi_{\text {trig. }}$. Of the 151 systems, 93 are from previous compendia of proper motion stars, primarily based on work by Luyten (Luyten 1979a, 1979b, 1980a, 1980b), and 58 are from our SuperCOSMOS-RECONS (SCR) search (Hambly et al. 2004; Henry et al. 2004). The systems have $\mu=118-828 \mathrm{mas} \mathrm{yr}^{-1}$, with 143 having $\mu>$

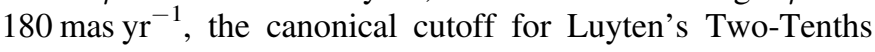
(LTT) Catalog.

We define M dwarfs to have $3.7 \leqslant(V-K) \leqslant 9.5$ and $8.8 \leqslant$ $M_{V} \leqslant 20.0$, corresponding to objects with masses $0.64-0.075$ $M_{\odot}$ (Benedict et al. 2016). For the most massive M dwarfs, these color and absolute magnitude limits were determined at the $\mathrm{K} / \mathrm{M}$ dwarf boundary by creating observational HR diagrams from the RECONS 25 Parsec Database (see Section 7), where spectral types from RECONS' work, Gray et al. (2003), Reid et al. (1995), and Hawley et al. (1996) were used to separate the $\mathrm{K}$ and $\mathrm{M}$ dwarfs. Because spectral types can be imprecise, there was overlap between the two spectral types; boundaries were thus chosen to split the types at carefully defined $(V-K)$ and $M_{V}$ values. A similar method was followed for the $\mathrm{M} / \mathrm{L}$ dwarf transition using results from both RECONS and Dahn et al. (2002), providing the regimes outlined above for the observational luminosities and colors that include the full range of $\mathrm{M}$ dwarfs. Stars for which astrometry and photometry are presented here have $3.85<(V-K)<8.47$ and are therefore all M dwarfs.

Figure 1 provides some of the general characteristics of the stars presented here. The left panel shows the distribution on the sky for the sample (note that there are eight stars with $\delta>0^{\circ}$ ). The right panel illustrates an observational luminosity function, using $M_{V}$, for the sample, indicating that the majority of these $\mathrm{M}$ dwarfs are of the mid-M spectral type. The true distribution would shift slightly toward lower mass stars with the deblending of the photometry of the 21 unresolved binaries (indicated in blue).

\section{OBSERVATIONS AND DATA REDUCTION}

\subsection{Photometry}

\subsection{1. $V_{J} R_{K C} I_{K C} C C D$ Photometry}

For all but two objects, ${ }^{10}$ at least two epochs of absolute $V_{J} R_{K C} I_{K C}$ (henceforth referred to as $V R I$ ) photometry on the Johnson-Kron-Cousins system were measured for each parallax field. Two $V$ filters that are photometrically indistinguishable to 7 millimagnitudes (mmag) (Jao et al. 2011), one $R$ filter, and one $I$ filter were used for series of observations spanning 2-16 years, depending on the star. ${ }^{11}$ The $2048 \times 2046$ Tektronix CCD camera on the CTIO/SMARTS $0.9 \mathrm{~m}$ with a pixel (px) scale of $0 . " 401 \mathrm{px}^{-1}$ was used for both astrometric and photometric observations. In order to mitigate the effects of image distortion at the edges of the CCD, only the central quarter of the chip was used, resulting in a $6 ! 8 \times 6 ! 8$ square field of view.

The data were reduced using $I R A F^{12}$ (Tody 1993). Bias subtraction and flat fielding were done with calibration frames acquired nightly. Most of the targets were observed at sec $z<1.8$, although for a few of the more extreme northern stars, this was not possible. Standard stars from Bessel (1990), Landolt (1992, 2007, 2013), and/or Graham (1982) were observed multiple times throughout the photometry nights at varying airmasses and used to determine transformation equations and extinction curves. Apertures $14^{\prime \prime}$ in diameter were used to determine the stellar fluxes, matching the aperture diameters used by Landolt. In cases where close contaminating sources required deblending, smaller apertures were used and aperture corrections were applied.

\footnotetext{
${ }^{10}$ Only one epoch of photometry was available for WT 1637 and LHS 2024 at the time of this paper.

11 The central wavelengths for the two $V_{J}$ filters, the $R_{\mathrm{KC}}$ filter, and the $I_{\mathrm{KC}}$ filter are $5438 \AA, 5475 \AA, 6425 \AA$, and $8075 \AA$, respectively.

12 IRAF is distributed by the National Optical Astronomy Observatories, which are operated by the Association of Universities for Research in Astronomy, Inc., under cooperative agreement with the National Science Foundation.
} 
A few systems had separations too small to allow aperture corrections; thus, point-spread function (PSF) photometry was performed using either IRAF or IDL. For SCR 1630-3633AB, the standard IRAF PSF reduction procedures ${ }^{13}$ were followed to obtain individual photometry for the components of these close binaries. For all other PSF reductions, a custom IDL program was used. In short, the contribution from the sky background was calculated from a "blank" part of the image. The region around the close pair being analyzed was then cropped to include only the relevant pair, and the background subtracted. A Moffat curve was fit to the PSF of the primary, the flux determined from the fit, and then the primary was subtracted from the image. Care was taken to minimize the residual counts from the primary. Gaussian and Lorentzian curves were also tested, but it was found that the Moffat curve fit the shape of the PSF best. A Moffat curve was then fit to the secondary component's PSF and the flux calculated from the fit. The ratio of the fluxes then yielded the $\Delta$ mags from which individual magnitudes were calculated from the blended photometry. VRI photometry from PSF reductions listed in Table 1 are noted.

As outlined in Winters et al. (2011), photometric uncertainties are typically $30 \mathrm{mmag}$ for the $V$-band and $20 \mathrm{mmag}$ for the $R$ - and $I$-bands. Further details on the photometric data reduction, the transformation equations, uncertainties, etc., can be found in Jao et al. $(2005,2011)$ and Winters et al. (2011, 2015). Note that a "u" next to the reference in column (7) indicates that photometry was reported in a previous RECONS publication, but that the value listed here is updated due to the acquisition of additional data.

\subsection{2. $\mathrm{JHK}_{s}$ Photometry from $2 \mathrm{MASS}$}

Photometry in the near-infrared $J H K_{s}$ (hereafter simply $J H K$ ) filters has been extracted from the Two Micron All Sky Survey (2MASS; Skrutskie et al. 2006) and is rounded to the nearest hundredth magnitude in Table 1 . Uncertainties in $J H K$ are typically less than $50 \mathrm{mmag}$, with exceptions indicated in columns $(8,9,10)$ of Table 1.

All JHK magnitudes have been confirmed by eye to correspond to the correct objects by using the Aladin interface at $C D S$ to "blink" SuperCOSMOS images taken at different epochs and identifying the 2MASS point that matches the projected proper motion. Because the proper motions of all the objects presented here are larger than 118 mas yr $^{-1}$, their movements were easily discernible during the blinking process and the correct identification of the 2MASS source was straightforward.

Table 1 provides the VRIJHK photometry data for the 160 stars in $151 \mathrm{M}$ dwarf systems presented here. Included are the names of the M dwarfs (column 1), coordinates (J2000.0) (2, $3), V R I$ magnitudes $(4,5,6)$ and the number of observations and/or reference (7), the 2MASS $J H K$ magnitudes $(8,9,10)$, and a photometric distance estimate and uncertainty based on the photometry $(11,12)$. Components of multiple systems are noted with capital letters $\mathrm{A}$ and $\mathrm{B}$ after the names in the first column. If the names of the components are different, the letters identifying the primary and the secondary are placed within parentheses (e.g., LTT 7419(A) and CE 507(B), where the "B" component is also known as LTT 7419B). A "J" next

\footnotetext{
${ }^{13}$ As outlined in A User's Guide to Stellar CCD Photometry with IRAF from NOAO.
}

to a magnitude indicates that light from a close companion has resulted in blended photometry. A note has been added to the photometric distance estimates for the 27 systems for which the trigonometric distance places the system beyond $25 \mathrm{pc}$. Nearly all of these stars were originally thought to be within $25 \mathrm{pc}$, but our new astrometry indicates that they are not. This in itself reveals important information about the stars, as discussed in Section 4.1.

\subsection{Astrometry}

Here we briefly outline the methods used for astrometry observations and data reduction. Parallax frames are taken using either $V_{J}$ (old), $V_{J}$ (new $)^{14}, R_{\mathrm{KC}}$, or $I_{\mathrm{KC}}$ filters, depending on the brightness of the science target, using the same instrumental set-up as that used for the photometry frames. The magnitudes of the stars span $7.94 \leqslant V R I \leqslant 18.55$. To balance the signal from the red target stars to generally fainter, bluer, reference stars, bright stars were observed in $V$, while to increase the signal for faint target stars, the $I$ filter was used. This reduces the magnitude range for target star observations in the parallax filters to $9.74 \leqslant V R I \leqslant 15.91$.

Typically, three to five frames (depending on the target's brightness) were taken each night within 30 minutes of the science target's meridian transit to minimize corrections required for differential color refraction (DCR). The parallax star and the reference stars do not have identical colors; therefore, their positions shift relative to each other as a result of refraction by the Earth's atmosphere, necessitating the DCR corrections. Mean exposure times ranged from 20 to $1200 \mathrm{~s}$, depending on the brightnesses of the science stars, the reference field stars, and sky conditions. All data were taken between 1999 and 2016, with timespans for observations for each star given in column (8) of Table 2. As with the photometry frames, bias and dome flat frames were taken nightly to enable calibration of the science images, following the procedures outlined in Jao et al. (2005).

As discussed extensively in Jao et al. (2005), Henry et al. (2006), and Subasavage et al. (2009), our data reduction processes disentangle the proper motions and parallactic shifts that contribute to the paths traced on the sky by the science targets. In short, (1) SExtractor (Bertin \& Arnouts 1996) was used to measure the centroids of the science and reference stars, (2) a six-constant plate model was defined to determine plate constants in order to rotate and translate each frame so that the reference stars were aligned from frame to frame, (3) leastsquare equations were then solved for multi-epoch series of images using Gaussfit (Jefferys et al. 1988), and (4) the relative parallax was corrected to absolute parallax via the photometric distance estimates of the reference stars, which were initially assumed to have zero mean parallax and proper motion.

As noted in Subasavage et al. (2009), in 2007, our reduction processes began incorporating a new centroiding algorithm available in the SExtractor package (see the SExtractor v2.13

\footnotetext{
${ }^{14}$ Between 2005 March and 2009 September, the $V_{J}$ (new) filter was used in lieu of the $V_{J}$ (old) filter and was found to be slightly inferior, astrometrically; however, as noted in Subasavage et al. (2009), there is a benefit to including data taken with both filters if there are $\sim 1-2$ years of data taken after the filter switch. See Subasavage et al. (2009) and Jao et al. (2011) for a more thorough discussion. Of the targets presented here, 62 were observed in the $V$ filter; all but two-L 749-34 and LHS 3124-have either all of their observations in the $V_{J}$ (old) filter or the necessary 1-2 years of additional data after the filter switch. Neither L 749-34 nor LHS 3124 show any signs of perturbation, so it is not felt that the astrometry has been affected.
} 
Table 1

Photometric Data

\begin{tabular}{|c|c|c|c|c|c|c|c|c|c|c|c|}
\hline $\begin{array}{l}\text { Name } \\
\text { (1) }\end{array}$ & $\begin{array}{c}\text { R.A. } \\
\text { (hh:mm:ss) } \\
\text { (2) }\end{array}$ & $\begin{array}{c}\text { Decl. } \\
\text { (dd:mm:ss) } \\
\text { (3) }\end{array}$ & $\begin{array}{c}V_{J} \\
(\mathrm{mag}) \\
(4)\end{array}$ & $\begin{array}{c}R_{\mathrm{KC}} \\
(\mathrm{mag}) \\
(5)\end{array}$ & $\begin{array}{c}I_{\mathrm{KC}} \\
(\mathrm{mag}) \\
(6)\end{array}$ & $\begin{array}{c}\text { \# Obs/Ref } \\
\text { (7) }\end{array}$ & $\begin{array}{c}J \\
(\mathrm{mag}) \\
(8)\end{array}$ & $\begin{array}{c}H \\
(\mathrm{mag}) \\
(9)\end{array}$ & $\begin{array}{c}K_{s} \\
(\mathrm{mag}) \\
(10)\end{array}$ & $\begin{array}{l}d_{\mathrm{CCD}} \\
(\mathrm{pc}) \\
(11)\end{array}$ & $\begin{array}{r}\sigma_{\mathrm{CCD}} \\
(\mathrm{pc}) \\
(12)\end{array}$ \\
\hline L 217-028 & $0008 \quad 17.4$ & -570553 & 12.13 & 11.00 & 9.57 & $/ 3$ & 8.21 & 7.63 & 7.40 & 13.2 & 2.1 \\
\hline LEHPM 1-255 & 000945.1 & -420140 & 13.62 & 12.35 & 10.66 & $/ 3$ & 9.07 & 8.45 & 8.22 & 12.5 & 2.0 \\
\hline SCR 0027-0806 & 002745.4 & -080605 & 17.54 & 15.84 & 13.73 & $3 / 2 \mathrm{u}$ & 11.57 & 10.97 & 10.61 & $18.4^{\mathrm{a}}$ & 2.9 \\
\hline LP $349-25 \mathrm{AB}$ & 002756.0 & +221933 & $18.04 \mathrm{~J}$ & $15.82 \mathrm{~J}$ & $13.37 \mathrm{~J}$ & $3 / 1$ & $10.61 \mathrm{~J}$ & 9.97J & $9.57 \mathrm{~J}$ & $8.2^{\mathrm{b}}$ & 1.3 \\
\hline LTT 313 & 003538.1 & -100419 & 11.85 & 10.81 & 9.57 & $/ 3$ & 8.33 & 7.74 & 7.48 & $17.5^{\mathrm{a}}$ & 2.7 \\
\hline LHS 1134 & 004326.0 & -411734 & 13.00 & 11.75 & 10.11 & $/ 3$ & 8.57 & 8.03 & 7.71 & 10.9 & 1.7 \\
\hline LHS 1140 & 004459.3 & -151618 & 14.18 & 12.88 & 11.19 & $/ 3$ & 9.61 & 9.09 & 8.82 & 17.1 & 2.9 \\
\hline L $87-2$ & 005712.5 & -641524 & 12.40 & 11.28 & 9.81 & $/ 3$ & 8.40 & 7.82 & 7.60 & 13.7 & 2.1 \\
\hline L $87-10$ & 010407.0 & -652227 & 13.98 & 12.63 & 10.95 & $/ 3$ & 9.40 & 8.84 & 8.53 & 14.7 & 2.5 \\
\hline LP 707-16 & 011017.5 & -115118 & 12.69 & 11.50 & 10.02 & $/ 3$ & 8.63 & 7.93 & 7.66 & 12.8 & 2.2 \\
\hline SCR 0111-4908 & 011147.5 & -490809 & 17.74 & 15.91 & 13.75 & $3 / 2 u$ & 11.54 & 11.00 & 10.61 & 17.5 & 2.8 \\
\hline LEHPM 1-1343 & 011316.4 & -542914 & 14.16 & 12.85 & 11.17 & $/ 3$ & 9.57 & 8.98 & 8.68 & 15.1 & 2.3 \\
\hline L $2-60$ & 012920.9 & -855611 & 13.49 & 12.21 & 10.58 & $/ 3$ & 9.05 & 8.39 & 8.08 & $12.2^{\mathrm{a}}$ & 2.0 \\
\hline WT 50 & 014629.4 & -533933 & 14.18 & 12.82 & 11.08 & $/ 3$ & 9.46 & $8.85^{\mathrm{c}}$ & 8.61 & 13.7 & 2.3 \\
\hline L 294-92AB & 014742.6 & -483606 & $12.42 \mathrm{~J}$ & $11.23 \mathrm{~J}$ & $9.72 \mathrm{~J}$ & $/ 3$ & $8.25 \mathrm{~J}$ & $7.69 \mathrm{~J}$ & $7.45 \mathrm{~J}$ & $11.5^{\mathrm{b}}$ & 1.8 \\
\hline LHS 5045 & 015251.6 & -480541 & 13.79 & 12.48 & 10.80 & $/ 3$ & 9.17 & $8.60^{\mathrm{c}}$ & 8.24 & 12.0 & 1.9 \\
\hline L $88-43$ & 015337.1 & -665334 & 11.68 & 10.60 & 9.24 & $/ 3$ & 7.91 & 7.29 & 6.99 & 11.6 & 1.9 \\
\hline L 583-33B & $0202 \quad 17.5$ & -263352 & 14.28 & 13.03 & 11.50 & $2 / 1$ & 9.94 & 9.32 & $9.06^{\mathrm{c}}$ & $20.9^{\mathrm{a}}$ & 3.4 \\
\hline L 583-33A & $\begin{array}{lll}02 & 02 & 17.5\end{array}$ & -263352 & 14.15 & 12.92 & 11.39 & $2 / 1$ & $9.81^{\mathrm{c}}$ & $9.22^{\mathrm{c}}$ & 8.95 & $20.0^{\mathrm{a}}$ & 3.3 \\
\hline SCR 0211-0354 & $02 \quad 1151.7$ & -035402 & 15.99 & 14.43 & 12.54 & $3 / 1$ & 10.68 & 10.07 & 9.73 & $16.4^{\mathrm{a}}$ & 2.3 \\
\hline L $225-57 \mathrm{AB}$ & 023421.2 & -530537 & $12.53 \mathrm{~J}$ & $11.32 \mathrm{~J}$ & $9.76 \mathrm{~J}$ & $/ 3$ & $8.23 \mathrm{~J}$ & $7.61 \mathrm{~J}$ & $7.31 \mathrm{~J}$ & $9.5^{\mathrm{b}}$ & 1.5 \\
\hline SCR 0246-7024 & 024602.3 & -702406 & 14.86 & 13.44 & 11.61 & $/ 2$ & 9.84 & 9.33 & 9.02 & 14.2 & 2.3 \\
\hline LP $831-45 A B$ & $\begin{array}{lll}03 & 14 & 18.2\end{array}$ & -230930 & $12.58 \mathrm{~J}$ & $11.42 \mathrm{~J}$ & $9.93 \mathrm{~J}$ & $/ 3$ & $8.49 \mathrm{~J}$ & $7.88 \mathrm{~J}$ & $7.63 \mathrm{~J}$ & $12.8^{\mathrm{b}}$ & 2.0 \\
\hline LP 831-68 & 031647.8 & -212526 & 11.04 & 10.01 & 8.79 & $/ 3$ & 7.61 & 6.95 & 6.69 & 12.5 & 2.1 \\
\hline LTT 17505 & 032146.9 & -064024 & 11.32 & 10.29 & 9.07 & $/ 3$ & 7.86 & 7.28 & 6.98 & 14.4 & 2.3 \\
\hline SCR 0327-3634 & 032746.8 & -363440 & 12.72 & 11.62 & 10.25 & $3 / 3 u$ & 8.92 & 8.26 & 8.04 & $18.4^{\mathrm{a}}$ & 3.0 \\
\hline SCR 0420-7005 & 042012.6 & -700559 & 17.09 & 15.35 & 13.25 & $/ 2$ & 11.19 & 10.59 & 10.25 & 16.3 & 2.7 \\
\hline L $130-37$ & 042805.7 & -620925 & 13.06 & 11.88 & 10.36 & $/ 3$ & 8.94 & $8.34^{\mathrm{c}}$ & 8.01 & 14.9 & 2.4 \\
\hline L 591-43(B) & 043639.8 & -272206 & 12.56 & 11.47 & 10.10 & $2 / 1$ & 8.78 & 7.17 & 7.88 & $14.9^{\mathrm{a}}$ & 5.4 \\
\hline L 591-42(A) & 043640.9 & $-2721 \quad 18$ & 12.25 & 11.19 & 9.88 & $/ 3$ & 8.61 & 7.98 & 7.73 & 17.9 & 2.8 \\
\hline LP 715-39 & 043837.2 & -113015 & 12.69 & 11.54 & 10.09 & $/ 3$ & 8.67 & 8.11 & 7.82 & 14.9 & 2.3 \\
\hline SCR $0509-4325$ & 050943.9 & $\begin{array}{lll}-43 & 2517\end{array}$ & 14.10 & 12.83 & 11.20 & $3 / 2 \mathrm{u}$ & 9.61 & 9.00 & 8.74 & 16.5 & 2.5 \\
\hline SCR $0517-4252$ & 051721.4 & -425247 & 11.92 & 10.87 & 9.61 & $/ 3$ & 8.34 & $7.73^{\mathrm{c}}$ & 7.45 & 16.3 & 2.6 \\
\hline LHS 5109 & 053600.1 & -073859 & 12.86 & 11.62 & 10.05 & $/ 3$ & 8.46 & 7.83 & 7.57 & 10.0 & 1.6 \\
\hline LEHPM 2-3528 & 060758.1 & -611510 & 14.57 & 13.26 & 11.57 & $/ 2$ & 9.92 & $9.39^{\mathrm{c}}$ & 9.09 & $18.0^{\mathrm{a}}$ & 2.8 \\
\hline SCR 0631-8811 & 063131.0 & -881137 & 15.65 & 14.05 & 12.04 & $/ 2$ & 10.04 & 9.46 & 9.07 & 10.4 & 1.6 \\
\hline SCR 0640-0552 & 064014.0 & -055223 & 10.22 & 9.22 & 8.03 & $/ 2$ & 6.84 & 6.21 & 5.96 & 9.3 & 1.5 \\
\hline SCR 0642-6707 & 064227.2 & -670720 & 16.01 & 14.43 & 12.42 & $/ 2$ & 10.62 & 10.15 & 9.81 & 17.6 & 3.9 \\
\hline L 597-18 & 064340.7 & -262441 & 12.92 & 11.76 & 10.31 & $/ 3$ & 8.91 & $8.43^{\mathrm{c}}$ & 8.13 & 18.1 & 3.0 \\
\hline SCR 0644-4223A & 064432.1 & -422345 & $14.74^{\mathrm{d}}$ & 13.47 & 11.95 & $1 / 2 \mathrm{u}$ & $9.93 \mathrm{~J}$ & $9.27 \mathrm{~J}$ & $8.98 \mathrm{~J}$ & $\ldots$ & $\ldots$ \\
\hline SCR 0644-4223B & 064432.1 & -422345 & $16.04^{\mathrm{d}}$ & 14.77 & 12.82 & $1 / 2 \mathrm{u}$ & $\ldots$ & $\ldots$ & $\ldots$ & $\ldots$ & $\ldots$ \\
\hline LP 382-56A & 065711.7 & -432451 & $11.89^{\mathrm{d}}$ & 10.85 & 9.47 & $1 / 3 u$ & $7.59 \mathrm{~J}$ & $6.98 \mathrm{~J}$ & $6.76 \mathrm{~J}$ & $\ldots$ & $\ldots$ \\
\hline LP 382-56B & 065711.7 & -432451 & $11.95^{\mathrm{d}}$ & 10.89 & 9.81 & $1 / 3 \mathrm{u}$ & $\ldots$ & $\ldots$ & $\ldots$ & $\ldots$ & $\ldots$ \\
\hline SCR 0702-6102 & 070250.4 & -610248 & 16.62 & 14.77 & 12.50 & $4 / 2 u$ & 10.36 & 9.85 & 9.52 & 10.7 & 2.0 \\
\hline APM 0710-5704 & 070937.7 & -570342 & 13.64 & 12.36 & 10.73 & $/ 3$ & 9.25 & $8.63^{\mathrm{c}}$ & 8.30 & 14.3 & 2.4 \\
\hline SCR 0713-0511 & $\begin{array}{lll}07 & 13 & 11.2\end{array}$ & -051149 & 11.13 & 10.08 & 8.86 & $/ 2$ & 7.65 & 7.08 & 6.82 & 13.5 & 2.1 \\
\hline SCR 0717-0501 & 071717.1 & -050104 & 13.29 & 12.02 & 10.39 & $/ 2$ & 8.87 & $8.35^{\mathrm{c}}$ & 8.05 & 13.2 & 2.2 \\
\hline LTT 17957 & 071732.2 & +193353 & 12.83 & 11.72 & 10.35 & $2 / 1$ & 9.02 & 8.43 & 8.16 & 19.9 & 3.1 \\
\hline L $136-37$ & 072052.0 & $\begin{array}{lll}-62 & 10 & 12\end{array}$ & 12.33 & 11.18 & 9.74 & $/ 3$ & 8.38 & $7.84^{\mathrm{c}}$ & 7.52 & 13.8 & 2.2 \\
\hline SCR $0723-8015 \mathrm{AB}$ & 072359.7 & -801518 & $17.45 \mathrm{~J}$ & $15.61 \mathrm{~J}$ & $13.40 \mathrm{~J}$ & $/ 3$ & $11.30 \mathrm{~J}$ & $10.82 \mathrm{~J}$ & $10.44 \mathrm{~J}$ & $17.1^{\mathrm{b}}$ & 3.2 \\
\hline SCR 0724-3125 & 072421.2 & -312558 & 14.40 & 13.07 & 11.39 & $3 / 3 u$ & 9.79 & 9.22 & 8.89 & $16.6^{\mathrm{a}}$ & 2.6 \\
\hline SCR 0733-4406 & 073342.7 & -440613 & 15.15 & 13.80 & 12.07 & $3 / 3 u$ & 10.32 & 9.73 & 9.44 & $18.4^{\mathrm{a}}$ & 2.9 \\
\hline SCR 0736-3024 & 073656.7 & -302416 & 13.67 & 12.42 & 10.85 & $3 / 2 \mathrm{u}$ & 9.36 & 8.79 & 8.50 & 16.9 & 2.7 \\
\hline L 528-16 & 073809.7 & $\begin{array}{lll}-31 & 12 & 19\end{array}$ & 11.90 & 10.80 & 9.48 & $/ 3$ & 8.18 & 7.56 & 7.30 & 14.0 & 2.2 \\
\hline SCR 0754-3809 & 075454.8 & -380938 & 15.46 & 13.91 & 11.98 & $/ 2$ & 10.01 & 9.42 & 9.08 & 11.3 & 1.8 \\
\hline SCR 0805-5912 & 080546.2 & -591250 & 14.69 & 13.38 & 11.68 & $/ 2$ & 10.07 & 9.52 & 9.22 & 19.4 & 3.1 \\
\hline LHS 1992 & 081342.6 & -760749 & 11.86 & 10.79 & 9.46 & $2 / 3 \mathrm{u}$ & 8.16 & $7.60^{\mathrm{c}}$ & 7.30 & 14.5 & 2.3 \\
\hline SCR 0829-3709 & 082941.3 & -370935 & 14.30 & 13.03 & 11.42 & $3 / 3 u$ & 9.83 & 9.26 & 9.00 & $19.2^{\mathrm{a}}$ & 3.0 \\
\hline LHS 2024 & 083123.5 & -102954 & 15.06 & 13.62 & 11.84 & $/ 3$ & 10.07 & 9.49 & 9.14 & 14.7 & 2.3 \\
\hline
\end{tabular}


Table 1

(Continued)

\begin{tabular}{|c|c|c|c|c|c|c|c|c|c|c|c|}
\hline Name & $\begin{array}{c}\text { R.A. } \\
\text { (hh:mm:ss) } \\
(2)\end{array}$ & $\begin{array}{c}\text { Decl. } \\
\text { (dd:mm:ss) } \\
(3)\end{array}$ & $\begin{array}{c}V_{J} \\
(\mathrm{mag}) \\
(4)\end{array}$ & $\begin{array}{c}R_{\mathrm{KC}} \\
(\mathrm{mag}) \\
(5)\end{array}$ & $\begin{array}{c}I_{\mathrm{KC}} \\
(\mathrm{mag}) \\
(6)\end{array}$ & $\begin{array}{c}\# \text { Obs/Ref } \\
\text { (7) }\end{array}$ & $\begin{array}{c}J \\
(\mathrm{mag}) \\
(8)\end{array}$ & $\begin{array}{c}H \\
(\mathrm{mag}) \\
(9)\end{array}$ & $\begin{array}{c}K_{s} \\
(\mathrm{mag}) \\
(10)\end{array}$ & $\begin{array}{l}d_{\mathrm{CCD}} \\
(\mathrm{pc}) \\
(11)\end{array}$ & $\begin{array}{l}\sigma_{\mathrm{CCD}} \\
(\mathrm{pc}) \\
(12)\end{array}$ \\
\hline SCR 0838-5855AB & 083802.2 & -585559 & $17.19 \mathrm{~J}$ & $15.08 \mathrm{~J}$ & $12.78 \mathrm{~J}$ & $/ 3$ & $10.31 \mathrm{~J}$ & $9.71 \mathrm{~J}$ & $9.27 \mathrm{~J}$ & $8.0^{\mathrm{b}}$ & $\overline{1.3}$ \\
\hline SCR 0853-3924 & 085328.7 & -392441 & 12.33 & 11.23 & 9.87 & $3 / 3 u$ & 8.51 & 7.94 & 7.72 & 16.4 & 2.5 \\
\hline L $532-12$ & 085402.4 & -305137 & 13.44 & 12.16 & 10.55 & $/ 3$ & 9.01 & 8.39 & 8.09 & $12.6^{\mathrm{a}}$ & 2.0 \\
\hline SCR 0914-4134 & 091417.4 & -413438 & 15.01 & 13.57 & 11.72 & $/ 2$ & 9.98 & 9.42 & 9.12 & 14.6 & 2.4 \\
\hline L 749-34 & 091620.7 & -183733 & 10.73 & 9.72 & 8.54 & $/ 3$ & 7.35 & 6.76 & 6.49 & 12.1 & 1.9 \\
\hline WT 1637 & 092903.8 & -242904 & 16.87 & 15.10 & 12.96 & $/ 3$ & 10.84 & 10.29 & 9.91 & 13.4 & 2.2 \\
\hline LP 788-1AB & 093122.3 & $-17 \quad 1743$ & $17.55 \mathrm{~J}$ & $15.63 \mathrm{~J}$ & $13.38 \mathrm{~J}$ & $/ 3$ & $11.07 \mathrm{~J}$ & $10.47 \mathrm{~J}$ & $10.07 \mathrm{~J}$ & $12.5^{\mathrm{b}}$ & 2.0 \\
\hline WT 2458 & 094558.4 & -325330 & 14.04 & 12.66 & 10.89 & $/ 3$ & 9.20 & 8.60 & 8.28 & 10.8 & 1.7 \\
\hline LTT 3613 & 095040.5 & -134839 & 12.71 & 11.52 & 9.99 & $/ 3$ & 8.58 & 7.97 & 7.72 & 13.2 & 2.1 \\
\hline L 392-39B & 101951.0 & -414848 & 13.17 & 12.00 & 10.49 & $2 / 1$ & 9.06 & 8.54 & 8.29 & 18.1 & 2.9 \\
\hline L $392-39 A$ & 101951.3 & -414846 & 11.64 & 10.66 & 9.52 & $/ 3$ & 8.36 & 7.72 & 7.49 & 20.0 & 3.2 \\
\hline LP 904-36 & 103628.4 & $-28 \quad 27 \quad 15$ & 12.03 & 10.99 & 9.75 & $2 / 3 u$ & 8.50 & $7.83^{c}$ & 7.58 & $17.5^{\mathrm{a}}$ & 3.0 \\
\hline LTT 3896 & 103745.3 & -274639 & 13.14 & 11.84 & 10.19 & $/ 3$ & 8.60 & $8.04^{c}$ & 7.72 & 10.1 & 1.6 \\
\hline LEHPM 2-2758 & 103847.8 & -863244 & 13.24 & 12.02 & 10.46 & $/ 3$ & 8.97 & 8.40 & 8.11 & 14.4 & 2.2 \\
\hline LP 848-50AB & 104241.4 & -241605 & $16.53 \mathrm{~J}$ & $14.60 \mathrm{~J}$ & $12.47 \mathrm{~J}$ & $3 / 3 u$ & $10.28 \mathrm{~J}$ & $9.67 \mathrm{~J}$ & $9.34 \mathrm{~J}$ & $9.7^{\mathrm{b}}$ & 1.6 \\
\hline LTT $4004 \mathrm{AB}$ & 105442.0 & -071833 & $13.29 \mathrm{~J}$ & $12.03 \mathrm{~J}$ & $10.43 \mathrm{~J}$ & $2 / 3 u$ & $8.88 \mathrm{~J}$ & $8.25 \mathrm{~J}$ & 7.97J & $12.2^{\mathrm{b}}$ & 1.9 \\
\hline LP 491-51 & 110321.3 & +133757 & 12.96 & 11.76 & 10.24 & $2 / 1$ & 8.76 & 8.18 & 7.91 & 13.7 & 2.1 \\
\hline CE440-87 & 114750.7 & -284945 & 16.21 & 14.98 & 13.38 & $/ 3$ & 12.02 & 11.45 & 11.24 & $67.9^{\mathrm{a}}$ & 14.0 \\
\hline SCR 1157-0149 & 115745.6 & -014903 & 15.99 & 14.54 & 12.68 & $/ 2$ & 10.91 & 10.35 & 10.02 & 21.3 & 3.4 \\
\hline LP 908-10 & 120328.1 & -292300 & 15.38 & 14.08 & 12.37 & $2 / 3 u$ & 10.74 & 10.13 & 9.81 & $24.2^{\mathrm{a}}$ & 3.7 \\
\hline SCR $1206-3500$ & 120658.5 & -350052 & 14.67 & 13.35 & 11.66 & $/ 2$ & 10.01 & 9.40 & 9.13 & 17.7 & 2.7 \\
\hline WT 1928 & 120806.9 & -320636 & 14.04 & 12.96 & 11.62 & $/ 3$ & 10.30 & 9.66 & 9.40 & $35.8^{\mathrm{a}}$ & 5.8 \\
\hline SCR 1214-4603 & 121440.0 & -460314 & 15.65 & 14.14 & 12.23 & $/ 2$ & 10.32 & 9.75 & 9.44 & 14.3 & 2.2 \\
\hline L $18-22$ & 122033.8 & -822558 & 11.96 & 10.76 & 9.20 & $/ 3$ & 7.70 & 7.14 & 6.84 & 8.1 & 1.2 \\
\hline SCR $1230-3411 \mathrm{AB}$ & 123001.8 & -341124 & $14.16 \mathrm{~J}$ & $12.81 \mathrm{~J}$ & $11.07 \mathrm{~J}$ & $/ 2$ & $9.34 \mathrm{~J}$ & $8.77 \mathrm{~J}^{\mathrm{c}}$ & $8.44 \mathrm{~J}$ & $11.7^{\mathrm{b}}$ & 1.8 \\
\hline L $327-121 \mathrm{AB}$ & 123333.1 & -482611 & $12.07 \mathrm{~J}$ & $10.91 \mathrm{~J}$ & $9.45 \mathrm{~J}$ & $2 / 3 \mathrm{u}$ & $8.01 \mathrm{~J}$ & $7.38 \mathrm{~J}$ & $7.14 \mathrm{~J}$ & $10.3^{\mathrm{b}}$ & 1.6 \\
\hline SCR $1240-8116$ & 124056.0 & -811631 & 14.11 & 12.89 & 11.28 & $/ 2$ & 9.73 & 9.16 & 8.89 & $19.2^{\mathrm{a}}$ & 2.9 \\
\hline LHS 5226 & 124400.7 & -111030 & 14.22 & 12.84 & 11.14 & $2 / 3 u$ & 9.52 & 8.97 & 8.67 & 14.6 & 2.4 \\
\hline SCR 1245-5506 & 124552.5 & -550650 & 13.66 & 12.32 & 10.61 & $/ 2$ & 8.99 & 8.43 & 8.12 & 11.3 & 1.8 \\
\hline LHS 2634 & 124709.8 & -033418 & 12.60 & 11.51 & 10.11 & $/ 3$ & 8.77 & 8.16 & 7.89 & 17.1 & 2.7 \\
\hline SCR $1247-0525$ & 124714.7 & -052513 & 14.78 & 13.45 & 11.73 & $/ 3$ & 10.13 & 9.62 & 9.29 & 20.1 & 3.4 \\
\hline LHS 5231 & 125918.2 & -001033 & 12.92 & 11.77 & 10.24 & $2 / 3 u$ & 8.79 & $8.23^{\mathrm{c}}$ & 7.92 & $14.4^{\mathrm{a}}$ & 2.2 \\
\hline WT $1962 \mathrm{AB}$ & 125951.3 & -073035 & $15.42 \mathrm{~J}$ & $14.23 \mathrm{~J}$ & $12.68 \mathrm{~J}$ & $/ 3$ & $11.19 \mathrm{~J}$ & $10.68 \mathrm{~J}$ & $10.43 \mathrm{~J}$ & $44.7^{\mathrm{a}, \mathrm{b}}$ & 7.2 \\
\hline LEP 1330-2039 & 133040.9 & -203903 & 12.46 & 11.27 & 9.75 & $/ 3$ & 8.28 & 7.65 & 7.39 & 10.7 & 1.7 \\
\hline SCR 1343-4002 & 134341.5 & -400229 & 15.06 & 13.67 & 11.88 & $2 / 3 u$ & 10.14 & 9.61 & 9.25 & 16.4 & 2.5 \\
\hline L $106-15$ & 140408.2 & -661437 & 11.59 & 10.51 & 9.14 & $/ 3$ & 7.78 & 7.15 & 6.92 & 11.0 & 1.8 \\
\hline SCR 1420-7516 & 142036.8 & -751606 & 13.78 & 12.55 & 10.95 & $/ 2$ & 9.44 & 8.91 & 8.63 & 17.9 & 2.9 \\
\hline LHS 2935 & 143208.0 & +081130 & 15.68 & 14.08 & 12.09 & $3 / 1$ & 10.11 & 9.53 & 9.17 & 11.2 & 1.8 \\
\hline SCR 1441-7338 & 144114.4 & -733841 & 16.96 & 15.31 & 13.25 & $/ 2$ & 11.20 & 10.61 & 10.27 & $17.2^{\mathrm{a}}$ & 2.7 \\
\hline SCR $1444-3426$ & 144406.6 & -342647 & 14.17 & 12.89 & 11.26 & $/ 2$ & 9.74 & 9.18 & 8.88 & 18.8 & 3.0 \\
\hline LTT 6084 & 151543.7 & -072521 & 12.93 & 11.68 & 10.09 & $2 / 3 u$ & 8.57 & 8.02 & 7.71 & 11.4 & 1.8 \\
\hline L $108-87$ & 153052.0 & -680118 & 13.52 & 12.28 & 10.63 & $/ 3$ & 9.08 & 8.50 & 8.23 & 13.6 & 2.1 \\
\hline L $408-123 \mathrm{AB}$ & 154541.6 & -433029 & $13.06 \mathrm{~J}$ & $11.90 \mathrm{~J}$ & $10.40 \mathrm{~J}$ & $/ 3$ & $8.94 \mathrm{~J}$ & $8.34 \mathrm{~J}$ & $8.08 \mathrm{~J}$ & $15.5^{\mathrm{b}}$ & 2.4 \\
\hline LHS 3124 & 155121.0 & +293106 & 13.03 & 11.87 & 10.39 & $2 / 1$ & 8.96 & 8.38 & 8.15 & 16.9 & 2.6 \\
\hline SCR 1601-3421 & 160155.7 & -342157 & 16.18 & 14.71 & 12.86 & $/ 2$ & 10.96 & 10.33 & 9.98 & 18.7 & 3.0 \\
\hline SCR $1626-3812$ & 162651.7 & -381233 & 15.75 & 14.24 & 12.29 & $/ 3$ & 10.37 & 9.80 & 9.44 & 13.8 & 2.1 \\
\hline SCR $1630-3633$ A & 163027.2 & -363356 & $15.35^{\mathrm{d}}$ & 13.83 & 11.92 & $1 / 1$ & $10.04 \mathrm{~J}$ & $9.50 \mathrm{~J}$ & $9.03 \mathrm{~J}$ & $\ldots$ & $\ldots$ \\
\hline SCR $1630-3633 B$ & 163027.2 & -363356 & $19.98^{\mathrm{d}}$ & 19.17 & 18.26 & $1 / 1$ & $\ldots$ & $\ldots$ & $\ldots$ & $\ldots$ & $\ldots$ \\
\hline SCR $1636-4041$ & 163657.6 & -404109 & 13.44 & 12.24 & 10.70 & $2 / 3 u$ & 9.20 & 8.57 & 8.31 & 15.7 & 2.5 \\
\hline LP 154-205 & 164755.2 & -650912 & 13.98 & 12.73 & 11.16 & $/ 3$ & 9.70 & 9.17 & 8.88 & 21.2 & 3.6 \\
\hline G $169-29$ & 165057.0 & +222648 & 14.08 & 12.69 & 10.91 & $3 / 1$ & 9.14 & 8.57 & 8.31 & 10.5 & 1.6 \\
\hline SCR 1656-2046 & 165633.6 & -204637 & 17.32 & 15.55 & 13.39 & $2 / 3 u$ & 11.30 & 10.71 & 10.37 & 16.6 & 2.8 \\
\hline G $139-3 \mathrm{AB}$ & 165825.0 & +135806 & $13.16 \mathrm{~J}$ & $11.90 \mathrm{~J}$ & $10.31 \mathrm{~J}$ & $3 / 1$ & $8.80 \mathrm{~J}$ & $8.28 \mathrm{~J}$ & $7.97 \mathrm{~J}$ & $13.1^{\mathrm{b}}$ & 2.1 \\
\hline SCR 1659-6958 & 165928.1 & -695818 & 15.36 & 13.93 & 12.15 & $/ 3$ & 10.54 & 10.00 & 9.70 & 22.2 & 4.3 \\
\hline SCR 1728-0143 & 172811.1 & -014357 & 14.42 & 13.15 & 11.51 & $/ 3$ & 9.89 & 9.32 & 9.01 & 18.2 & 2.8 \\
\hline SCR $1731-2452$ & 173103.8 & -245244 & 13.20 & 12.07 & 10.63 & $2 / 1$ & 9.27 & 8.61 & 8.38 & $19.8^{\mathrm{a}}$ & 3.2 \\
\hline L $204-148$ & 173832.5 & -583234 & 12.52 & 11.38 & 9.91 & $/ 3$ & 8.48 & 7.93 & 7.67 & 13.9 & 2.1 \\
\hline LTT 7077 & 174629.4 & -084237 & 12.72 & 11.44 & 9.78 & $/ 3$ & 8.20 & 7.69 & 7.35 & 8.8 & 1.4 \\
\hline SCR $1746-3214$ & 174640.7 & -321404 & 16.15 & 14.47 & 12.40 & $/ 3$ & 10.35 & 9.74 & 9.38 & 11.2 & 1.8 \\
\hline
\end{tabular}


Table 1

(Continued)

\begin{tabular}{|c|c|c|c|c|c|c|c|c|c|c|c|}
\hline $\begin{array}{l}\text { Name } \\
\text { (1) }\end{array}$ & $\begin{array}{c}\text { R.A. } \\
\text { (hh:mm:ss) } \\
(2)\end{array}$ & $\begin{array}{c}\text { Decl. } \\
\text { (dd:mm:ss) } \\
\text { (3) }\end{array}$ & $\begin{array}{c}V_{J} \\
(\mathrm{mag}) \\
(4)\end{array}$ & $\begin{array}{c}R_{\mathrm{KC}} \\
(\mathrm{mag}) \\
(5)\end{array}$ & $\begin{array}{c}I_{\mathrm{KC}} \\
(\mathrm{mag}) \\
(6)\end{array}$ & $\begin{array}{c}\# \text { Obs/Ref } \\
\text { (7) }\end{array}$ & $\begin{array}{c}J \\
(\mathrm{mag}) \\
(8)\end{array}$ & $\begin{array}{c}H \\
(\mathrm{mag}) \\
(9)\end{array}$ & $\begin{array}{c}K_{s} \\
(\mathrm{mag}) \\
(10)\end{array}$ & $\begin{array}{c}d_{\mathrm{CCD}} \\
(\mathrm{pc}) \\
(11)\end{array}$ & $\begin{array}{l}\sigma_{\mathrm{CCD}} \\
(\mathrm{pc}) \\
(12)\end{array}$ \\
\hline L 415-82 & 175714.2 & -415929 & 11.28 & 10.30 & 9.17 & $/ 3$ & 7.96 & 7.37 & 7.14 & 16.9 & 2.7 \\
\hline G $154-43 \mathrm{AB}$ & 180336.1 & -185851 & $14.13 \mathrm{~J}$ & $12.72 \mathrm{~J}$ & $10.90 \mathrm{~J}$ & $/ 3$ & $9.13 \mathrm{~J}$ & $8.53 \mathrm{~J}$ & $8.28 \mathrm{~J}$ & $9.9^{\mathrm{b}}$ & 1.6 \\
\hline L $43-72 \mathrm{AB}$ & 181115.3 & -785923 & $12.57 \mathrm{~J}$ & $11.23 \mathrm{~J}$ & $9.50 \mathrm{~J}$ & $/ 3$ & $7.84 \mathrm{~J}$ & $7.33 \mathrm{~J}$ & $6.96 \mathrm{~J}$ & $6.4^{\mathrm{b}}$ & 1.0 \\
\hline LTT 7246A & 181512.4 & -192407 & $11.02^{\mathrm{d}}$ & 10.03 & 8.96 & $1 / 3 \mathrm{u}$ & $7.32 \mathrm{~J}$ & $6.72 \mathrm{~J}$ & $6.45 \mathrm{~J}$ & $\ldots$ & $\ldots$ \\
\hline SCR 1826-6542 & 182646.8 & -654240 & 17.35 & 15.28 & 12.96 & $/ 2$ & 10.57 & 9.96 & 9.55 & 9.3 & 1.5 \\
\hline GJ 714 & 183012.0 & -581628 & 9.81 & 8.90 & 7.93 & $/ 3$ & 6.87 & 6.24 & 5.96 & 11.9 & 2.0 \\
\hline G $141-21$ & 183619.0 & +133630 & 12.45 & 11.23 & 9.69 & $3 / 1$ & 8.19 & 7.61 & 7.37 & 10.4 & 1.6 \\
\hline SCR 1841-4347 & 184109.8 & -434733 & 16.46 & 14.72 & 12.59 & $/ 2$ & 10.48 & 9.94 & 9.60 & 11.9 & 2.0 \\
\hline LTT 7419(A) & 184312.5 & -332246 & 10.25 & 9.30 & 8.28 & $/ 3$ & 7.19 & 6.54 & 6.33 & 13.3 & 2.2 \\
\hline CE 507(B) & 184312.7 & -332226 & 16.24 & 14.66 & 12.67 & $2 / 1$ & 10.73 & 10.14 & 9.83 & 15.7 & 2.5 \\
\hline LTT 7434AB & 184557.5 & -285553 & $12.68 \mathrm{~J}$ & $11.49 \mathrm{~J}$ & $9.94 \mathrm{~J}$ & $/ 3$ & $8.41 \mathrm{~J}$ & $7.90 \mathrm{~J}$ & $7.60 \mathrm{~J}$ & $11.6^{\mathrm{b}}$ & 1.8 \\
\hline L 345-91B & 184841.4 & -464708 & $12.45^{\mathrm{d}}$ & 11.33 & 10.06 & $1 / 3 \mathrm{u}$ & $\ldots$ & $\ldots$ & $\ldots$ & $\ldots$ & $\ldots$ \\
\hline L $345-91 \mathrm{~A}$ & 184841.4 & -464708 & $12.26^{\mathrm{d}}$ & 11.18 & 9.72 & $1 / 3 u$ & $7.83 \mathrm{~J}$ & $7.24 \mathrm{~J}$ & $6.99 \mathrm{~J}$ & $\ldots$ & $\ldots$ \\
\hline SIP 1848-8214 & 184851.1 & -821442 & 18.55 & 16.33 & 14.00 & $/ 3$ & 11.48 & 10.92 & 10.50 & 13.8 & 2.2 \\
\hline LP 691-15 & 184906.4 & -031518 & 14.23 & 12.91 & 11.22 & $/ 3$ & 9.61 & 8.98 & 8.70 & 14.8 & 2.3 \\
\hline L 489-43 & 185225.3 & -373036 & 12.70 & 11.49 & 9.86 & $/ 3$ & 8.38 & 7.86 & 7.56 & 11.1 & 1.9 \\
\hline SCR 1855-6914 & 185547.9 & $-6914 \quad 15$ & 16.61 & 14.79 & 12.66 & $/ 2$ & 10.47 & 9.88 & 9.51 & 10.7 & 1.7 \\
\hline SCR 1901-0737 & 190132.4 & -073724 & 15.59 & 14.16 & 12.35 & $2 / 3 u$ & 10.57 & 10.00 & 9.70 & $19.0^{\mathrm{a}}$ & 3.0 \\
\hline SCR 1901-3106 & 190159.2 & -310645 & 13.37 & 12.29 & 10.93 & $2 / 3 \mathrm{u}$ & 9.61 & 9.01 & 8.77 & $27.2^{\mathrm{a}}$ & 4.2 \\
\hline LHS 5348AB & 192752.7 & $-2811 \quad 15$ & $14.85 \mathrm{~J}$ & $13.36 \mathrm{~J}$ & $11.46 \mathrm{~J}$ & $/ 3$ & $9.69 \mathrm{~J}$ & $9.11 \mathrm{~J}$ & $8.79 \mathrm{~J}$ & $11.6^{\mathrm{b}}$ & 1.9 \\
\hline SCR 1931-0306 & 193104.6 & -030618 & 16.81 & 15.11 & 13.11 & $/ 2$ & 11.15 & 10.56 & 10.23 & 18.0 & 3.0 \\
\hline L $275-26$ & 193403.9 & -522514 & 12.83 & 11.68 & 10.19 & $/ 3$ & 8.77 & 8.16 & 7.92 & 15.0 & 2.3 \\
\hline LHS 3492 & 195131.3 & -505538 & 15.27 & 14.05 & 12.45 & $/ 3$ & 10.97 & 10.49 & 10.19 & $38.8^{\mathrm{a}}$ & 6.8 \\
\hline SCR 2016-7531 & 201611.3 & -753105 & 15.84 & 14.28 & 12.35 & $/ 2$ & 10.47 & 9.86 & 9.51 & 14.3 & 2.3 \\
\hline SCR 2025-1534 & 202508.6 & -153416 & 12.70 & 11.62 & 10.25 & $/ 3$ & 8.92 & 8.30 & 8.05 & $19.0^{\mathrm{a}}$ & 3.0 \\
\hline LEHPM 2-1265AB & 203301.9 & -490311 & $15.33 \mathrm{~J}$ & $13.84 \mathrm{~J}$ & $11.98 \mathrm{~J}$ & $/ 3$ & $10.11 \mathrm{~J}$ & $9.52 \mathrm{~J}$ & $9.19 \mathrm{~J}$ & $13.3^{\mathrm{b}}$ & 2.1 \\
\hline LP567-63 & 203431.1 & -323100 & 12.17 & 11.08 & 9.71 & $/ 3$ & 8.37 & 7.74 & 7.51 & $14.6^{\mathrm{a}}$ & 2.3 \\
\hline L $163-23$ & 210225.0 & -603136 & 11.96 & 10.97 & 9.79 & $/ 3$ & 8.64 & 7.92 & 7.71 & $21.0^{\mathrm{a}}$ & 3.7 \\
\hline LHS 3615 & 210321.7 & -502252 & 13.10 & 11.96 & 10.48 & $/ 3$ & 9.11 & 8.46 & 8.19 & $17.4^{\mathrm{a}}$ & 2.8 \\
\hline SCR 2105-5503 & 210513.8 & -550356 & 13.97 & 12.69 & 11.05 & $/ 2$ & 9.59 & 8.92 & 8.64 & 16.7 & 2.8 \\
\hline LEHPM 2-801 & 212216.9 & -431405 & 13.39 & 12.17 & 10.61 & $/ 2$ & 9.13 & 8.53 & 8.21 & 14.9 & 2.3 \\
\hline L 714-46 & 212818.3 & -221832 & 12.17 & 11.11 & 9.81 & $/ 3$ & 8.50 & 7.92 & 7.63 & 16.9 & 2.7 \\
\hline SCR 2130-7710 & 213007.0 & -771038 & 17.01 & 15.34 & 13.31 & $/ 2$ & 11.29 & 10.67 & 10.37 & 18.3 & 2.9 \\
\hline L 2-77 & 214640.4 & -854305 & 13.39 & 12.13 & 10.44 & $/ 3$ & 8.84 & 8.28 & 7.99 & 11.4 & 1.8 \\
\hline L 427-34B & 214911.4 & -413330 & $13.44^{\mathrm{d}}$ & 12.16 & 10.78 & $2 / 1$ & $\ldots$ & $\ldots$ & $\ldots$ & $\ldots$ & $\ldots$ \\
\hline L $427-34 \mathrm{~A}$ & 214911.4 & -413330 & $13.14^{\mathrm{d}}$ & 11.82 & 10.39 & $2 / 1$ & $7.75 \mathrm{~J}$ & $7.17 \mathrm{~J}$ & $6.88 \mathrm{~J}$ & $\ldots$ & $\ldots$ \\
\hline LEHPM 1-4771AB & 223009.5 & -534456 & $14.47 \mathrm{~J}$ & $13.09 \mathrm{~J}$ & $11.30 \mathrm{~J}$ & $/ 3$ & $9.54 \mathrm{~J}$ & $8.96 \mathrm{~J}$ & $8.63 \mathrm{~J}$ & $12.0^{\mathrm{b}}$ & 1.8 \\
\hline L 645-73(B) & 223823.0 & -292100 & 12.64 & 11.46 & 9.96 & $5 / 1$ & 8.57 & 7.99 & 7.70 & 13.7 & 2.2 \\
\hline SCR 2303-4650 & 230335.6 & -465047 & 13.89 & 12.54 & 10.83 & $2 / 1$ & 9.22 & 8.69 & 8.26 & 12.0 & 2.0 \\
\hline SCR $2307-8452$ & $2307 \quad 19.7$ & -845204 & 15.15 & 13.76 & 12.00 & $3 / 2 \mathrm{u}$ & 10.36 & 9.81 & 9.47 & 19.7 & 3.3 \\
\hline L $85-31$ & 235325.2 & -705641 & 13.01 & 11.78 & 10.18 & $/ 3$ & 8.68 & 8.10 & 7.78 & 11.8 & 1.8 \\
\hline LTT $9828 \mathrm{AB}$ & 235944.8 & -440500 & $12.81 \mathrm{~J}$ & $11.67 \mathrm{~J}$ & $10.23 \mathrm{~J}$ & $/ 3$ & $8.88 \mathrm{~J}$ & $8.29 \mathrm{~J}$ & $8.04 \mathrm{~J}$ & $17.6^{\mathrm{b}}$ & 2.7 \\
\hline
\end{tabular}

Notes. A " $\mathrm{J}$ " next to a photometry value indicates that the magnitude is blended due to one or more close companions. A "u" next to the reference indicates that RECONS photometry was reported in a previous publication, but that the value listed here is updated due to additional data and supersedes previous results.

References. (1) This work, (2) Winters et al. (2011), (3) Winters et al. (2015).

a The $\pi_{\text {trig }}$ for this object places it beyond $25 \mathrm{pc}$.

${ }^{\mathrm{b}}$ The photometric distance estimate is based upon the joint photometry for the system and is thus likely an underestimate.

c 2 MASS magnitude uncertainty greater than $50 \mathrm{mmag}$.

${ }^{\mathrm{d}}$ VRI photometry for individual components has been calculated using PSF reduction.

User's manual ${ }^{15}$ for a more thorough discussion) that utilizes a circular Gaussian-weighted two-dimensional window to iteratively measure the pixel coordinates of the reference and parallax stars. The $x$ and $y$ pixel coordinates are measured through the XWIN_IMAGE and YWIN_IMAGE outputs. The

\footnotetext{
$\overline{15}$ https://www.astromatic.net/pubsvn/software/sextractor/trunk/doc/ sextractor.pdf
}

resulting positional parameters are much more precise than those obtained using the isophotal method and are very close to the precision obtained using a PSF-fitting model.

The final $\pi_{\text {trig }}$ uncertainty is a combination of many factors, including (1) the quality of the reference star frame (number of stars, distribution, brightness), (2) the accuracy of the $(x, y)$ centroids, including any ellipticity caused by a close component, (3) the total number of images used in the $\pi_{\text {trig }}$ 
Table 2

Astrometry Data

\begin{tabular}{|c|c|c|c|c|c|c|c|c|c|c|c|c|c|c|c|c|}
\hline Name & $\begin{array}{c}\text { R.A. } \\
\text { (hh:mm:ss) } \\
\text { (2) }\end{array}$ & $\begin{array}{c}\text { Decl. } \\
\text { (dd:mm:ss) } \\
\text { (3) }\end{array}$ & Filt & $\begin{array}{l}N_{\text {sea }} \\
\text { (5) }\end{array}$ & $N_{\text {fr }}$ & $\begin{array}{c}\text { Coverage } \\
\text { (7) }\end{array}$ & $\begin{array}{c}T \\
\text { (year) } \\
(8)\end{array}$ & $\begin{array}{l}N_{\text {ref }} \\
\text { (9) }\end{array}$ & $\begin{array}{c}\text { var } \\
(\mathrm{mmag}) \\
(10)\end{array}$ & $\begin{array}{c}\pi_{\text {rel }} \\
(\text { mas) } \\
(11)\end{array}$ & $\begin{array}{c}\pi_{\text {cor }} \\
\text { (mas) } \\
(12)\end{array}$ & $\begin{array}{c}\pi_{\text {abs }} \\
\text { (mas) } \\
(13)\end{array}$ & $\begin{array}{c}\mu \\
\left(\operatorname{mas~yr}^{-1}\right) \\
(14)\end{array}$ & $\begin{array}{l}\text { P.A. } \\
\text { (deg) } \\
(15)\end{array}$ & $\begin{array}{c}V_{\text {tan }} \\
\left(\mathrm{km} \mathrm{s}^{-1}\right) \\
(16)\end{array}$ & $\begin{array}{l}\text { Notes } \\
\text { (17) }\end{array}$ \\
\hline L $217-28$ & $0008 \quad 17.37$ & -570552.9 & $\mathrm{~V}$ & $8 \mathrm{~s}$ & 68 & $2000.50-2008.00$ & 7.25 & 7 & 9.8 & $73.66 \pm 2.10$ & $1.51 \pm 0.21$ & $75.17 \pm 2.11$ & $385.4 \pm 0.7$ & $263.1 \pm 0.17$ & 24.3 & \\
\hline LEHPM 1-255 & 000945.06 & -420139.6 & $\mathrm{~V}$ & $4 \mathrm{c}$ & 51 & 2009.75-2012.94 & 3.20 & 6 & 13.7 & $52.12 \pm 1.49$ & $1.14 \pm 0.22$ & $53.26 \pm 1.51$ & $254.8 \pm 1.4$ & $091.4 \pm 0.44$ & 22.7 & \\
\hline SCR 0027-0806 & 002745.36 & -080604.7 & I & $5 \mathrm{~s}$ & 59 & 2008.85-2014.94 & 6.09 & 9 & 13.3 & $23.39 \pm 1.24$ & $1.50 \pm 0.10$ & $24.89 \pm 1.24$ & $118.5 \pm 0.9$ & $118.6 \pm 0.80$ & 22.6 & \\
\hline LP 349-25AB & 002755.99 & +221932.3 & I & $12 \mathrm{~s}$ & 100 & 2003.51-2015.92 & 12.42 & 7 & 11.2 & $67.71 \pm 1.39$ & $1.45 \pm 0.14$ & $69.16 \pm 1.40^{\mathrm{a}}$ & $429.8 \pm 0.3$ & $112.4 \pm 0.07$ & 29.5 & $!$ \\
\hline LTT 313 & 003538.07 & -100418.7 & V & $6 \mathrm{~s}$ & 70 & 2010.97-2015.97 & 5.00 & 7 & 9.9 & $31.14 \pm 1.32$ & $1.38 \pm 0.08$ & $34.52 \pm 1.32^{\mathrm{a}}$ & $211.1 \pm 1.0$ & $254.4 \pm 0.46$ & 30.1 & $!$ \\
\hline LHS 1134 & 004326.01 & $-41 \quad 1734.0$ & V & $7 \mathrm{~s}$ & 72 & 2009.78-2015.97 & 6.19 & 8 & 10.5 & $94.68 \pm 1.40$ & $0.56 \pm 0.08$ & $95.24 \pm 1.40$ & $764.4 \pm 0.8$ & $220.1 \pm 0.11$ & 38.0 & \\
\hline LHS 1140 & 004459.34 & $-1516 \quad 17.5$ & $\mathrm{~V}$ & $8 \mathrm{~s}$ & 60 & 2003.95-2014.93 & 10.98 & 5 & 12.5 & $79.57 \pm 2.76$ & $0.61 \pm 0.11$ & $80.18 \pm 2.76$ & $665.9 \pm 1.0$ & $153.3 \pm 0.16$ & 39.4 & \\
\hline L $87-2$ & 005712.48 & -641524.0 & $\mathrm{~V}$ & $5 \mathrm{~s}$ & 70 & $2008.86-2012.87$ & 4.01 & 7 & 8.9 & $55.57 \pm 1.38$ & $0.68 \pm 0.05$ & $56.25 \pm 1.38$ & $385.8 \pm 1.2$ & $061.6 \pm 0.33$ & 32.5 & \\
\hline L $87-10$ & 010406.95 & -652227.3 & $\mathrm{~V}$ & $8 \mathrm{~s}$ & 59 & 2008.64-2015.82 & 7.19 & 6 & 18.1 & $85.06 \pm 1.47$ & $0.45 \pm 0.04$ & $85.51 \pm 1.47$ & $365.8 \pm 0.6$ & $242.2 \pm 0.19$ & 20.3 & \\
\hline LP 707-16 & 011017.53 & -115117.6 & $\mathrm{~V}$ & $5 \mathrm{~s}$ & 56 & $2010.97-2014.93$ & 3.96 & 6 & 13.5 & $53.42 \pm 1.47$ & $0.52 \pm 0.07$ & $53.94 \pm 1.47$ & $230.0 \pm 1.4$ & $120.6 \pm 0.66$ & 20.2 & \\
\hline SCR 0111-4908 & 011147.52 & -490808.9 & $\mathrm{R}$ & $5 \mathrm{~s}$ & 59 & 2007.82-2014.92 & 7.09 & 8 & 11.2 & $52.96 \pm 1.39$ & $1.30 \pm 0.10$ & $54.26 \pm 1.39$ & $540.6 \pm 1.0$ & $215.0 \pm 0.21$ & 47.2 & \\
\hline LEHPM 1-1343 & 011316.41 & -542913.8 & $\mathrm{R}$ & $10 \mathrm{~s}$ & 80 & $1999.91-2014.80$ & 14.89 & 9 & 24.2 & $59.27 \pm 1.15$ & $1.61 \pm 0.18$ & $60.88 \pm 1.16^{\mathrm{a}}$ & $391.2 \pm 0.2$ & $072.3 \pm 0.05$ & 30.7 & \\
\hline L 2-60 & 012920.84 & -855611.0 & I & $6 s$ & 55 & $2003.95-2008.70$ & 4.75 & 10 & 6.7 & $30.71 \pm 2.47$ & $1.81 \pm 0.17$ & $32.52 \pm 2.48$ & $434.7 \pm 1.5$ & $138.1 \pm 0.39$ & 63.4 & \\
\hline WT 50 & 014629.36 & -533932.6 & $\mathrm{~V}$ & $5 \mathrm{~s}$ & 45 & 2011.61-2015.84 & 4.23 & 7 & 12.3 & $55.70 \pm 1.75$ & $0.96 \pm 0.22$ & $56.66 \pm 1.76$ & $178.7 \pm 1.1$ & $124.7 \pm 0.72$ & 14.9 & \\
\hline L 294-92AB & 014742.55 & -483605.7 & $\mathrm{R}$ & $12 \mathrm{~s}$ & 92 & 2004.74-2015.83 & 11.10 & 6 & 11.7 & $71.41 \pm 1.63$ & $0.92 \pm 0.08$ & $72.33 \pm 1.63$ & $364.2 \pm 0.5$ & $143.9 \pm 0.14$ & 23.9 & $!$ \\
\hline LHS 5045 & 015251.59 & -480541.3 & $\mathrm{R}$ & $12 \mathrm{~s}$ & 95 & 2003.95-2015.93 & 11.98 & 5 & 14.0 & $73.53 \pm 1.91$ & $1.60 \pm 0.09$ & $75.13 \pm 1.91$ & $550.9 \pm 0.5$ & $248.8 \pm 0.09$ & 34.8 & \\
\hline L $88-43$ & 015337.08 & -665334.1 & $\mathrm{R}$ & $7 \mathrm{~s}$ & 79 & 2005.71-2011.89 & 6.18 & 6 & 8.1 & $72.47 \pm 3.50$ & $2.97 \pm 0.24$ & $75.44 \pm 3.51$ & $424.2 \pm 1.4$ & $066.1 \pm 0.36$ & 26.7 & $!$ \\
\hline L 583-33A & $0202 \quad 17.53$ & -263351.4 & I & $7 \mathrm{~s}$ & 56 & $2000.57-2011.89$ & 11.32 & 6 & 17.7 & $33.05 \pm 1.29$ & $1.91 \pm 0.20$ & $34.96 \pm 1.31$ & $340.5 \pm 0.4$ & $143.6 \pm 0.14$ & 46.2 & \\
\hline L 583-33B & $0202 \quad 17.53$ & -263351.4 & I & $7 \mathrm{~s}$ & 56 & $2000.57-2011.89$ & 11.32 & 6 & 14.3 & $32.66 \pm 1.29$ & $1.91 \pm 0.20$ & $34.57 \pm 1.31$ & $336.0 \pm 0.4$ & $143.7 \pm 0.14$ & 46.1 & \\
\hline SCR 0211-0354 & 021151.67 & -035402.5 & $\mathrm{R}$ & $4 \mathrm{c}$ & 46 & 2011.73-2014.92 & 3.19 & 9 & 15.2 & $29.17 \pm 1.89$ & $1.21 \pm 0.13$ & $30.38 \pm 1.89$ & $152.2 \pm 1.5$ & $185.4 \pm 0.88$ & 23.7 & \\
\hline L 225-57AB & 023421.21 & -530536.8 & $\mathrm{~V}$ & $7 \mathrm{~s}$ & 61 & 2003.95-2010.97 & 7.02 & 8 & 11.2 & $50.09 \pm 1.82$ & $0.85 \pm 0.17$ & $50.94 \pm 1.83$ & $403.7 \pm 0.8$ & $145.2 \pm 0.23$ & 37.6 & \\
\hline SCR 0246-7024 & 024602.25 & -702406.3 & $\mathrm{R}$ & $9 \mathrm{~s}$ & 66 & 2006.87-2014.91 & 8.05 & 7 & 12.5 & $79.22 \pm 1.27$ & $0.96 \pm 0.08$ & $80.18 \pm 1.27$ & $225.4 \pm 0.5$ & $116.7 \pm 0.26$ & 13.3 & \\
\hline LP 831-45AB & 031418.18 & -230929.5 & $\mathrm{~V}$ & $13 \mathrm{~s}$ & 93 & 2003.95-2016.05 & 12.10 & 8 & 15.3 & $61.98 \pm 1.22$ & $1.53 \pm 0.14$ & $63.51 \pm 1.23^{\mathrm{a}}$ & $368.8 \pm 0.4$ & $062.6 \pm 0.11$ & 27.5 & $!$ \\
\hline LP 831-68 & 031647.81 & -212525.9 & $\mathrm{~V}$ & $5 \mathrm{~s}$ & 65 & 2010.96-2014.94 & 3.99 & 7 & 17.4 & $52.07 \pm 2.27$ & $1.39 \pm 0.59$ & $53.46 \pm 2.35$ & $285.2 \pm 1.3$ & $059.8 \pm 0.52$ & 25.3 & \\
\hline LTT 17505 & 032146.92 & -064024.3 & $\mathrm{~V}$ & $4 \mathrm{c}$ & 59 & 2012.82-2015.82 & 3.01 & 7 & 11.6 & $53.76 \pm 2.47$ & $1.10 \pm 0.15$ & $54.86 \pm 2.47$ & $312.1 \pm 2.3$ & $090.9 \pm 0.60$ & 27.0 & \\
\hline SCR 0327-3634 & 032746.79 & -363440.4 & $\mathrm{~V}$ & $4 c$ & 56 & 2011.77-2015.08 & 3.31 & 9 & 12.1 & $37.57 \pm 1.62$ & $0.56 \pm 0.07$ & $38.13 \pm 1.62$ & $183.0 \pm 1.6$ & $249.0 \pm 0.90$ & 22.8 & \\
\hline SCR 0420-7005 & 042012.55 & -700558.7 & I & $11 \mathrm{~s}$ & 92 & 2003.94-2015.82 & 11.89 & 7 & 7.1 & $59.19 \pm 0.74$ & $2.42 \pm 0.23$ & $61.61 \pm 0.77$ & $658.2 \pm 0.2$ & $021.6 \pm 0.04$ & 50.6 & \\
\hline L $130-37$ & 042805.72 & -620925.1 & $\mathrm{~V}$ & $5 \mathrm{~s}$ & 72 & 2011.73-2016.05 & 4.32 & 6 & 16.8 & $58.02 \pm 1.15$ & $1.40 \pm 0.15$ & $59.42 \pm 1.16$ & $358.3 \pm 0.9$ & $030.0 \pm 0.26$ & 28.6 & \\
\hline L 591-43(B) & 043639.83 & -272206.0 & $\mathrm{~V}$ & $5 \mathrm{~s}$ & 49 & $2000.96-2007.91$ & 4.19 & 5 & 8.7 & $38.03 \pm 2.59$ & $0.95 \pm 0.09$ & $38.98 \pm 2.59$ & $340.9 \pm 1.3$ & $237.8 \pm 0.43$ & 41.5 & $!$ \\
\hline L 591-42(A) & 043640.85 & $-2721 \quad 18.3$ & $\mathrm{~V}$ & $5 \mathrm{~s}$ & 49 & $2000.96-2007.91$ & 4.19 & 5 & 11.1 & $41.07 \pm 2.48$ & $0.95 \pm 0.09$ & $42.02 \pm 2.48$ & $343.1 \pm 1.3$ & $237.7 \pm 0.41$ & 38.7 & $!$ \\
\hline LP 715-39 & 043837.16 & -113014.8 & $\mathrm{~V}$ & $4 \mathrm{c}$ & 70 & 2010.96-2013.92 & 2.96 & 7 & 13.9 & $49.25 \pm 2.13$ & $1.70 \pm 0.20$ & $50.95 \pm 2.14$ & $360.7 \pm 2.3$ & $228.7 \pm 0.73$ & 33.6 & \\
\hline SCR 0509-4325 & 050943.85 & $\begin{array}{lll}-43 & 25 & 17.4\end{array}$ & I & $6 s$ & 71 & 2008.86-2015.05 & 6.20 & 8 & 18.2 & $44.79 \pm 1.82$ & $1.74 \pm 0.11$ & $46.53 \pm 1.82$ & $227.2 \pm 1.4$ & $324.2 \pm 0.69$ & 23.1 & \\
\hline SCR 0517-4252 & 051721.43 & -425247.3 & $\mathrm{~V}$ & $5 \mathrm{~s}$ & 64 & 2011.17-2014.92 & 3.76 & 9 & 13.1 & $46.31 \pm 1.55$ & $1.29 \pm 0.21$ & $47.60 \pm 1.56$ & $183.7 \pm 1.4$ & $011.2 \pm 0.73$ & 18.3 & \\
\hline LHS 5109 & 053600.07 & -073858.5 & V & $5 \mathrm{~s}$ & 70 & 2011.73-2016.06 & 4.32 & 11 & 11.6 & $66.94 \pm 1.51$ & $1.07 \pm 0.19$ & $68.01 \pm 1.52$ & $480.5 \pm 1.2$ & $013.5 \pm 0.24$ & 33.5 & \\
\hline LEHPM 2-3528 & 060758.09 & -611510.5 & I & $6 \mathrm{~s}$ & 58 & 2009.92-2015.96 & 6.03 & 8 & 8.3 & $22.94 \pm 1.61$ & $1.34 \pm 0.14$ & $24.28 \pm 1.62$ & $215.4 \pm 0.9$ & $005.8 \pm 0.36$ & 42.1 & \\
\hline SCR 0631-8811 & 063131.04 & -881136.6 & I & $8 \mathrm{c}$ & 131 & 2003.94-2016.05 & 12.11 & 9 & 13.3 & $60.66 \pm 2.06$ & $2.65 \pm 0.19$ & $63.31 \pm 2.07$ & $501.8 \pm 0.4$ & $354.0 \pm 0.06$ & 37.6 & \\
\hline SCR 0640-0552 & 064013.99 & -055223.3 & V & $9 \mathrm{c}$ & 122 & 2004.91-2012.82 & 7.90 & 10 & 11.5 & $83.20 \pm 2.96$ & $2.55 \pm 0.26$ & $85.75 \pm 2.97$ & $590.5 \pm 1.0$ & $170.0 \pm 0.16$ & 32.6 & \\
\hline SCR 0642-6707 & 064227.17 & -670719.8 & I & $8 \mathrm{~s}$ & 101 & 2006.87-2016.21 & 9.34 & 11 & 12.2 & $72.03 \pm 1.03$ & $1.35 \pm 0.08$ & $73.38 \pm 1.03$ & $819.5 \pm 0.6$ & $121.9 \pm 0.08$ & 52.0 & \\
\hline L 597-18 & 064340.67 & -262440.9 & $\mathrm{R}$ & $6 \mathrm{~s}$ & 114 & $2000.88-2014.16$ & 13.29 & 10 & 10.8 & $54.38 \pm 0.99$ & $1.05 \pm 0.04$ & $55.43 \pm 0.99$ & $463.3 \pm 0.3$ & $224.7 \pm 0.08$ & 39.6 & \\
\hline SCR 0644-4223A & 064432.09 & -422345.2 & I & $6 \mathrm{~s}$ & 33 & 2008.85-2013.78 & 4.93 & 11 & $\ldots$ & $29.98 \pm 2.79$ & $2.13 \pm 0.16$ & $32.11 \pm 2.79$ & $183.6 \pm 2.7$ & $162.2 \pm 1.46$ & 27.1 & \\
\hline SCR 0644-4223B & 064432.09 & -422345.2 & I & $6 \mathrm{~s}$ & 33 & $2008.85-2013.78$ & 4.93 & 11 & $\cdots$ & $33.14 \pm 3.05$ & $2.13 \pm 0.16$ & $35.27 \pm 3.05$ & $171.4 \pm 2.9$ & $165.1 \pm 1.72$ & 23.0 & \\
\hline LP 382-56A & 065711.74 & -432451.1 & $\mathrm{~V}$ & $4 c$ & 62 & 2010.96-2014.15 & 3.19 & 12 & $\ldots$ & $47.00 \pm 2.05$ & $1.28 \pm 0.11$ & $48.28 \pm 2.05$ & $255.2 \pm 2.1$ & $162.8 \pm 0.81$ & 25.1 & \\
\hline LP 382-56B & 065711.74 & -432451.1 & $\mathrm{~V}$ & $4 \mathrm{c}$ & 62 & 2010.96-2014.15 & 3.19 & 12 & $\cdots$ & $46.60 \pm 2.13$ & $1.28 \pm 0.11$ & $47.88 \pm 2.13$ & $220.0 \pm 2.1$ & $159.6 \pm 1.00$ & 21.8 & \\
\hline SCR 0702-6102 & 070250.34 & -610247.6 & I & $13 \mathrm{c}$ & 200 & 2003.84-2016.04 & 12.20 & 10 & 8.9 & $58.16 \pm 0.66$ & $1.29 \pm 0.15$ & $59.45 \pm 0.68$ & $785.7 \pm 0.1$ & $042.2 \pm 0.02$ & 62.7 & $!$ \\
\hline APM 0710-5704 & 070937.66 & -570342.3 & $\mathrm{R}$ & $4 c$ & 102 & 2000.14-2003.13 & 3.00 & 9 & 10.1 & $57.55 \pm 1.06$ & $1.55 \pm 0.18$ & $59.10 \pm 1.08$ & $464.8 \pm 1.3$ & $046.2 \pm 0.33$ & 37.3 & \\
\hline
\end{tabular}


Table 2

(Continued)

\begin{tabular}{|c|c|c|c|c|c|c|c|c|c|c|c|c|c|c|c|c|}
\hline Name & $\begin{array}{c}\text { R.A. } \\
\text { (hh:mm:ss) } \\
\text { (2) }\end{array}$ & $\begin{array}{c}\text { Decl. } \\
\text { (dd:mm:ss) } \\
\text { (3) }\end{array}$ & (4) & $\begin{array}{l}N_{\text {sea }} \\
\text { (5) }\end{array}$ & $N_{\text {fr }}$ & $\begin{array}{c}\text { Coverage } \\
\text { (7) }\end{array}$ & $\begin{array}{c}T \\
\text { (year) } \\
(8)\end{array}$ & $\begin{array}{l}N_{\text {ref }} \\
(9)\end{array}$ & $\begin{array}{c}\text { var } \\
(\mathrm{mmag}) \\
(10)\end{array}$ & $\begin{array}{c}\pi_{\text {rel }} \\
\text { (mas) } \\
(11)\end{array}$ & $\begin{array}{c}\pi_{\mathrm{cor}} \\
(\mathrm{mas}) \\
(12)\end{array}$ & $\begin{array}{c}\pi_{\mathrm{abs}} \\
(\mathrm{mas}) \\
(13)\end{array}$ & $\begin{array}{c}\mu \\
\left(\operatorname{mas~yr}^{-1}\right) \\
(14)\end{array}$ & $\begin{array}{l}\text { P.A. } \\
\text { (deg) } \\
(15)\end{array}$ & $\begin{array}{c}V_{\mathrm{tan}} \\
\left(\mathrm{km} \mathrm{s}^{-1}\right) \\
(16)\end{array}$ & $\begin{array}{c}\text { Notes } \\
\text { (17) }\end{array}$ \\
\hline SCR 0713-0511 & $07 \quad 13 \quad 11.23$ & -051148.6 & $\mathrm{~V}$ & $8 \mathrm{~s}$ & 71 & 2004.97-2012.89 & 7.92 & 11 & 13.5 & $89.66 \pm 1.64$ & $1.20 \pm 0.07$ & $90.86 \pm 1.64$ & $304.5 \pm 1.0$ & $184.1 \pm 0.29$ & 15.9 & \\
\hline SCR 0717-0501 & 071717.09 & -050103.6 & I & $11 \mathrm{~s}$ & 122 & $2004.18-2016.20$ & 12.02 & 9 & 14.5 & $91.69 \pm 1.66$ & $1.35 \pm 0.10$ & $93.04 \pm 1.66$ & $573.9 \pm 0.4$ & $132.8 \pm 0.07$ & 29.2 & \\
\hline LTT 17957 & 071729.94 & +193416.7 & $\mathrm{R}$ & $6 c$ & 73 & 2003.94-2009.02 & 5.08 & 8 & 7.7 & $51.97 \pm 2.38$ & $0.71 \pm 0.11$ & $52.68 \pm 2.38$ & $416.6 \pm 1.4$ & $230.6 \pm 0.37$ & 37.5 & \\
\hline L $136-37$ & 072052.04 & -621011.8 & I & $5 c$ & 58 & 2009.09-2012.89 & 3.81 & 8 & 9.8 & $87.85 \pm 2.56$ & $0.95 \pm 0.18$ & $88.80 \pm 2.57$ & $338.9 \pm 2.4$ & $303.5 \pm 0.80$ & 18.1 & \\
\hline SCR 0723-8015AB & 072359.65 & -80 1517.8 & I & $9 \mathrm{~s}$ & 113 & 2003.07-2016.18 & 13.11 & 8 & 7.9 & $63.21 \pm 1.15$ & $1.10 \pm 0.15$ & $64.31 \pm 1.16^{\mathrm{a}}$ & $818.9 \pm 0.2$ & $330.1 \pm 0.03$ & 60.4 & $!$ \\
\hline SCR $0724-3125$ & 072421.23 & -312557.7 & I & $5 \mathrm{~s}$ & 64 & 2011.23-2015.05 & 3.82 & 10 & 18.6 & $24.84 \pm 1.44$ & $1.15 \pm 0.08$ & $25.99 \pm 1.44$ & $135.2 \pm 1.1$ & $290.9 \pm 0.85$ & 24.7 & \\
\hline SCR 0733-4406 & 073342.67 & -440612.5 & I & $5 \mathrm{~s}$ & 64 & 2011.23-2014.95 & 3.71 & 10 & 10.4 & $22.45 \pm 0.73$ & $0.60 \pm 0.04$ & $23.05 \pm 0.73$ & $279.4 \pm 0.6$ & $165.3 \pm 0.23$ & 57.4 & \\
\hline SCR 0736-3024 & 073656.68 & -302416.4 & $\mathrm{R}$ & $7 \mathrm{~s}$ & 92 & $2006.87-2016.05$ & 9.18 & 12 & 12.8 & $73.78 \pm 1.00$ & $1.36 \pm 0.15$ & $75.14 \pm 1.01$ & $429.3 \pm 0.6$ & $148.5 \pm 0.15$ & 27.1 & \\
\hline L 528-16 & 073809.69 & -311219.1 & $\mathrm{~V}$ & $4 \mathrm{~s}$ & 63 & 2011.96-2015.22 & 3.26 & 8 & 12.3 & $65.97 \pm 1.31$ & $1.04 \pm 0.20$ & $67.01 \pm 1.33$ & $265.7 \pm 1.6$ & $320.6 \pm 0.69$ & 18.8 & \\
\hline SCR 0754-3809 & 075454.84 & -380937.8 & I & $11 \mathrm{~s}$ & 120 & 2004.97-2016.05 & 9.82 & 9 & 10.0 & $79.08 \pm 0.91$ & $1.39 \pm 0.22$ & $80.47 \pm 0.94$ & $371.6 \pm 0.3$ & $349.3 \pm 0.07$ & 21.9 & \\
\hline SCR 0805-5912 & 080546.17 & -591250.4 & I & $10 \mathrm{c}$ & 105 & 2003.94-2016.05 & 12.11 & 10 & 8.9 & $58.73 \pm 0.90$ & $1.75 \pm 0.20$ & $60.48 \pm 0.92$ & $632.8 \pm 0.2$ & $155.1 \pm 0.03$ & 49.6 & \\
\hline LHS 1992 & 081342.61 & -760748.5 & $\mathrm{~V}$ & $3 c$ & 62 & $2012.87-2015.29$ & 2.42 & 10 & 10.4 & $60.84 \pm 1.82$ & $1.02 \pm 0.12$ & $61.86 \pm 1.82$ & $696.9 \pm 2.3$ & $334.4 \pm 0.37$ & 53.4 & \\
\hline SCR 0829-3709 & 082941.29 & -370934.7 & $\mathrm{R}$ & $5 \mathrm{c}$ & 63 & $2011.16-2015.08$ & 3.93 & 11 & 13.8 & $38.26 \pm 0.87$ & $1.68 \pm 0.23$ & $39.94 \pm 0.90$ & $344.4 \pm 0.6$ & $304.2 \pm 0.21$ & 40.9 & \\
\hline LHS 2024 & 083123.45 & -102953.8 & I & $5 \mathrm{~s}$ & 68 & $2003.95-2016.21$ & 12.26 & 10 & 11.3 & $58.30 \pm 0.95$ & $1.11 \pm 0.06$ & $59.41 \pm 0.95$ & $671.3 \pm 0.3$ & $241.5 \pm 0.06$ & 53.6 & \\
\hline SCR $0838-5855 \mathrm{AB}$ & 083802.24 & -585558.7 & I & $9 \mathrm{~s}$ & 109 & $2005.90-2015.93$ & 10.03 & 11 & 10.5 & $91.38 \pm 0.75$ & $1.17 \pm 0.08$ & $92.55 \pm 0.75^{\mathrm{a}}$ & $330.9 \pm 0.3$ & $194.7 \pm 0.08$ & 16.6 & $!$ \\
\hline SCR 0853-3924 & 085328.65 & -392441.0 & $\mathrm{~V}$ & $5 \mathrm{c}$ & 65 & 2011.16-2014.95 & 3.79 & 11 & 13.2 & $57.76 \pm 1.23$ & $0.85 \pm 0.11$ & $58.61 \pm 1.23$ & $376.2 \pm 0.9$ & $265.7 \pm 0.22$ & 30.4 & \\
\hline L 532-12 & 085402.39 & -305136.6 & $\mathrm{~V}$ & $4 \mathrm{c}$ & 65 & 2012.94-2016.21 & 3.27 & 9 & 25.2 & $31.80 \pm 0.81$ & $1.26 \pm 0.20$ & $33.06 \pm 0.83$ & $273.2 \pm 0.7$ & $268.8 \pm 0.23$ & 39.2 & \\
\hline SCR 0914-4134 & 091417.42 & -413437.9 & I & $10 \mathrm{~s}$ & 109 & 2007.18-2016.05 & 8.87 & 10 & 8.0 & $82.23 \pm 0.68$ & $1.21 \pm 0.15$ & $83.44 \pm 0.70$ & $759.5 \pm 0.2$ & $314.5 \pm 0.04$ & 43.1 & \\
\hline L 749-34 & 091620.65 & -183732.9 & $\mathrm{~V}$ & $7 \mathrm{~s}$ & 73 & 2004.17-2009.94 & 5.76 & 9 & 10.9 & $75.60 \pm 2.36$ & $2.00 \pm 0.16$ & $77.60 \pm 2.37$ & $321.7 \pm 1.2$ & $296.0 \pm 0.41$ & 19.6 & \\
\hline WT 1637 & 092903.77 & -242904.3 & I & $4 \mathrm{~s}$ & 58 & 2013.38-2016.18 & 2.80 & 8 & 8.0 & $55.71 \pm 0.97$ & $0.84 \pm 0.04$ & $56.55 \pm 0.97$ & $251.5 \pm 1.1$ & $235.5 \pm 0.51$ & 21.1 & \\
\hline LP 788-1AB & 093122.27 & -171742.6 & I & $5 \mathrm{c}$ & 74 & 2011.97-2016.05 & 4.08 & 10 & 10.6 & $70.47 \pm 0.99$ & $0.83 \pm 0.06$ & $71.30 \pm 0.99^{\mathrm{a}}$ & $372.8 \pm 0.9$ & $246.2 \pm 0.25$ & 25.9 & $!$ \\
\hline WT 2458 & 094558.42 & -325329.8 & $\mathrm{R}$ & $7 \mathrm{~s}$ & 85 & 2010.39-2016.06 & 5.67 & 8 & 16.5 & $82.65 \pm 0.74$ & $0.71 \pm 0.10$ & $83.36 \pm 0.75$ & $327.7 \pm 0.4$ & $296.2 \pm 0.13$ & 18.6 & \\
\hline LTT 3613 & 095040.54 & -134838.6 & $\mathrm{~V}$ & $6 \mathrm{~s}$ & 59 & 2009.32-2013.95 & 4.63 & 10 & 11.1 & $49.66 \pm 1.86$ & $1.48 \pm 0.13$ & $51.14 \pm 1.86$ & $190.7 \pm 1.3$ & $122.8 \pm 0.74$ & 17.7 & \\
\hline L 392-39A & 101951.26 & -414846.1 & $\mathrm{~V}$ & $5 \mathrm{~s}$ & 62 & $2001.27-2005.10$ & 3.91 & 7 & 9.3 & $42.38 \pm 2.69$ & $2.15 \pm 0.22$ & $44.53 \pm 2.70$ & $446.1 \pm 1.1$ & $190.4 \pm 0.19$ & 47.5 & \\
\hline L 392-39B & 101953.66 & -414901.6 & $\mathrm{~V}$ & $5 \mathrm{~s}$ & 62 & $2001.27-2005.10$ & 3.91 & 7 & 6.9 & $44.48 \pm 2.60$ & $2.15 \pm 0.22$ & $46.63 \pm 2.61$ & $441.5 \pm 1.1$ & $190.5 \pm 0.26$ & 44.9 & \\
\hline LP 904-36 & 103628.43 & -28 2715.4 & $\mathrm{~V}$ & $6 \mathrm{~s}$ & 62 & $2010.02-2015.22$ & 5.20 & 8 & 14.2 & $35.88 \pm 1.51$ & $1.10 \pm 0.21$ & $36.98 \pm 1.52$ & $215.2 \pm 1.3$ & $269.6 \pm 0.48$ & 27.6 & \\
\hline LTT 3896 & 103745.32 & -274638.7 & $\mathrm{R}$ & $8 \mathrm{~s}$ & 96 & 2004.99-2016.04 & 11.05 & 7 & 15.5 & $65.30 \pm 1.28$ & $1.32 \pm 0.12$ & $66.62 \pm 1.29$ & $335.6 \pm 0.3$ & $320.6 \pm 0.09$ & 23.9 & $!$ \\
\hline LEHPM 2-2758 & 103847.82 & -863244.1 & $\mathrm{R}$ & $11 \mathrm{~s}$ & 99 & 2005.14-2016.05 & 10.91 & 8 & 9.6 & $73.35 \pm 2.54$ & $1.45 \pm 0.20$ & $74.80 \pm 2.55$ & $245.0 \pm 0.6$ & $227.0 \pm 0.29$ & 15.5 & \\
\hline LP 848-50AB & 104241.36 & -241604.9 & $\mathrm{R}$ & $7 \mathrm{~s}$ & 80 & $2010.16-2016.04$ & 5.88 & 13 & 14.8 & $93.62 \pm 1.30$ & $0.49 \pm 0.05$ & $94.11 \pm 1.30^{\mathrm{a}}$ & $209.4 \pm 1.0$ & $011.6 \pm 0.45$ & 11.2 & $!$ \\
\hline LTT 4004AB & 105441.97 & -071833.1 & $\mathrm{~V}$ & $4 \mathrm{c}$ & 50 & 2013.12-2016.19 & 3.07 & 6 & 13.0 & $46.26 \pm 1.89$ & $0.91 \pm 0.23$ & $47.17 \pm 1.90$ & $403.5 \pm 1.3$ & $205.6 \pm 0.37$ & 40.6 & \\
\hline LP 491-51 & 110321.27 & +133757.0 & $\mathrm{~V}$ & $7 \mathrm{~s}$ & 52 & $2010.15-2016.20$ & 6.05 & 6 & 13.3 & $63.88 \pm 2.63$ & $0.76 \pm 0.08$ & $64.64 \pm 2.63$ & $208.8 \pm 1.0$ & $294.3 \pm 0.52$ & 15.3 & \\
\hline CE $440-87$ & 114750.65 & -284944.7 & I & $5 \mathrm{~s}$ & 50 & 2001.14-2005.40 & 4.26 & 7 & 14.1 & $15.74 \pm 1.18$ & $0.69 \pm 0.06$ & $16.43 \pm 1.18$ & $386.2 \pm 0.9$ & $242.8 \pm 0.26$ & 111.4 & \\
\hline SCR 1157-0149 & 115745.55 & -014902.6 & I & $8 \mathrm{~s}$ & 70 & $2004.18-2012.28$ & 8.10 & 10 & 13.3 & $49.57 \pm 1.51$ & $1.19 \pm 0.13$ & $50.76 \pm 1.52$ & $459.9 \pm 0.6$ & $118.6 \pm 0.14$ & 42.9 & \\
\hline LP 908-10 & 120328.04 & -292300.3 & $\mathrm{R}$ & $4 \mathrm{c}$ & 46 & $2000.06-2014.44$ & 14.38 & 11 & $\cdots$ & $37.18 \pm 1.20$ & $0.46 \pm 0.06$ & $37.64 \pm 1.20$ & $314.0 \pm 0.2$ & $313.7 \pm 0.08$ & 39.5 & $!$ \\
\hline SCR 1206-3500 & 120658.52 & -350052.0 & I & $5 \mathrm{~s}$ & 58 & 2011.16-2015.24 & 4.08 & 8 & 9.5 & $40.74 \pm 1.41$ & $1.70 \pm 0.21$ & $42.44 \pm 1.43$ & $398.4 \pm 0.8$ & $227.4 \pm 0.23$ & 44.5 & \\
\hline WT 1928 & 120806.86 & -320636.1 & $\mathrm{~V}$ & $3 \mathrm{c}$ & 49 & $2000.14-2002.46$ & 2.32 & 10 & 8.0 & $16.92 \pm 1.21$ & $0.84 \pm 0.05$ & $17.76 \pm 1.21$ & $373.8 \pm 1.9$ & $261.1 \pm 0.47$ & 99.8 & \\
\hline SCR 1214-4603 & 121439.98 & -460314.3 & I & $7 \mathrm{~s}$ & 74 & 2008.13-2016.20 & 8.06 & 9 & 29.1 & $64.22 \pm 0.80$ & $1.58 \pm 0.09$ & $65.80 \pm 0.81$ & $765.6 \pm 0.3$ & $251.0 \pm 0.04$ & 55.2 & \\
\hline L $18-22$ & 122033.77 & -822557.8 & $\mathrm{~V}$ & $10 \mathrm{~s}$ & 123 & $2006.21-2015.39$ & 9.19 & 7 & 10.4 & $78.20 \pm 2.52$ & $1.50 \pm 0.50^{\mathrm{b}}$ & $79.70 \pm 2.57$ & $275.5 \pm 0.7$ & $007.7 \pm 0.23$ & 15.2 & \\
\hline SCR $1230-3411 \mathrm{AB}$ & 123001.75 & -341124.1 & $\mathrm{R}$ & $9 \mathrm{~s}$ & 77 & 2008.07-2016.19 & 8.11 & 8 & 10.3 & $52.85 \pm 0.99$ & $0.86 \pm 0.14$ & $53.71 \pm 1.00^{\mathrm{a}}$ & $517.8 \pm 0.5$ & $236.9 \pm 0.10$ & 45.7 & $!$ \\
\hline L $327-121 \mathrm{AB}$ & 123333.11 & $\begin{array}{lll}-48 & 26 & 11.2\end{array}$ & $\mathrm{~V}$ & $7 \mathrm{~s}$ & 58 & $2010.15-2016.06$ & 5.91 & 11 & 12.3 & $42.90 \pm 1.28$ & $1.18 \pm 0.14$ & $44.08 \pm 1.29^{\mathrm{a}}$ & $271.6 \pm 0.8$ & $291.5 \pm 0.31$ & 29.2 & \\
\hline SCR $1240-8116$ & 124056.02 & $\begin{array}{llll}-81 & 16 & 31.0\end{array}$ & $\mathrm{R}$ & $7 \mathrm{~s}$ & 62 & 2003.25-2011.16 & 7.92 & 9 & 8.2 & $29.56 \pm 2.21$ & $2.78 \pm 0.72$ & $32.34 \pm 2.32$ & $492.7 \pm 1.0$ & $280.4 \pm 0.19$ & 72.2 & \\
\hline LHS 5226 & 124400.73 & -111030.3 & $\mathrm{~V}$ & $6 \mathrm{~s}$ & 57 & 2009.32-2015.29 & 5.97 & 8 & 18.9 & $70.45 \pm 1.69$ & $1.83 \pm 0.16$ & $72.28 \pm 1.70$ & $503.6 \pm 0.9$ & $253.6 \pm 0.18$ & 33.0 & \\
\hline SCR 1245-5506 & 124552.53 & -550650.2 & I & $6 \mathrm{~s}$ & 55 & 2004.17-2009.08 & 4.91 & 5 & 8.6 & $97.82 \pm 1.35$ & $1.34 \pm 0.12$ & $99.16 \pm 1.36$ & $384.3 \pm 0.8$ & $111.4 \pm 0.21$ & 18.4 & \\
\hline LHS 2634 & 124709.78 & -033417.7 & $\mathrm{~V}$ & $4 \mathrm{~s}$ & 76 & $2001.15-2010.12$ & 8.97 & 5 & 13.8 & $45.03 \pm 1.97$ & $0.81 \pm 0.04$ & $45.84 \pm 1.97$ & $501.7 \pm 0.8$ & $269.1 \pm 0.13$ & 51.9 & \\
\hline SCR $1247-0525$ & 124714.73 & -052513.2 & I & $7 \mathrm{~s}$ & 67 & 2004.18-2011.42 & 7.24 & 9 & 8.9 & $48.53 \pm 1.06$ & $1.46 \pm 0.09$ & $49.99 \pm 1.06$ & $742.7 \pm 0.5$ & $320.3 \pm 0.07$ & 70.4 & \\
\hline
\end{tabular}


Table 2

(Continued)

\begin{tabular}{|c|c|c|c|c|c|c|c|c|c|c|c|c|c|c|c|c|}
\hline Name & $\begin{array}{c}\text { R.A. } \\
\text { (hh:mm:ss) } \\
\text { (2) }\end{array}$ & $\begin{array}{l}\text { Decl. } \\
\text { (dd:mm:ss) } \\
\text { (3) }\end{array}$ & Filt & $\begin{array}{l}N_{\text {sea }} \\
(5)\end{array}$ & $\begin{array}{l}N_{\text {fr }} \\
\text { (6) }\end{array}$ & $\begin{array}{c}\text { Coverage } \\
\text { (7) }\end{array}$ & $\begin{array}{c}T \\
\text { (year) } \\
(8)\end{array}$ & $\begin{array}{l}N_{\text {ref }} \\
\text { (9) }\end{array}$ & $\begin{array}{c}\text { var } \\
(\mathrm{mmag}) \\
(10)\end{array}$ & $\begin{array}{c}\pi_{\text {rel }} \\
(\mathrm{mas}) \\
(11)\end{array}$ & $\begin{array}{c}\pi_{\mathrm{cor}} \\
(\mathrm{mas}) \\
(12)\end{array}$ & $\begin{array}{c}\pi_{\mathrm{abs}} \\
(\mathrm{mas}) \\
(13)\end{array}$ & $\begin{array}{c}\mu \\
\left(\operatorname{mas~yr}^{-1}\right) \\
(14)\end{array}$ & $\begin{array}{l}\text { P.A. } \\
\text { (deg) } \\
(15)\end{array}$ & $\begin{array}{c}V_{\mathrm{tan}} \\
\left(\mathrm{km} \mathrm{s}^{-1}\right) \\
(16)\end{array}$ & Notes \\
\hline LHS 5231 & 125918.24 & -00 1033.4 & $\mathrm{~V}$ & $6 \mathrm{~s}$ & 65 & 2009.32-2015.30 & 5.98 & 8 & 10.5 & $32.31 \pm 1.73$ & $2.10 \pm 0.30$ & $34.41 \pm 1.76$ & $470.4 \pm 1.2$ & $161.2 \pm 0.27$ & 64.8 & \\
\hline WT $1962 \mathrm{AB}$ & 125951.26 & -073035.3 & I & $8 \mathrm{~s}$ & 91 & $2000.25-2011.50$ & 11.37 & 9 & 11.0 & $26.13 \pm 1.27$ & $0.75 \pm 0.12$ & $26.88 \pm 1.28^{\mathrm{a}}$ & $446.6 \pm 0.3$ & $295.4 \pm 0.07$ & 74.6 & $!$ \\
\hline LEP 1330-2039 & 133040.94 & -203903.4 & $\mathrm{~V}$ & $6 \mathrm{~s}$ & 70 & 2011.51-2016.19 & 4.69 & 8 & 13.9 & $58.40 \pm 1.52$ & $0.81 \pm 0.25$ & $59.21 \pm 1.54$ & $597.0 \pm 1.2$ & $148.7 \pm 0.22$ & 47.8 & \\
\hline SCR 1343-4002 & 134341.48 & -400229.3 & I & $4 \mathrm{c}$ & 75 & 2011.15-2014.43 & 3.28 & 9 & 8.4 & $57.46 \pm 0.99$ & $1.35 \pm 0.09$ & $58.81 \pm 0.99$ & $228.5 \pm 1.0$ & $114.2 \pm 0.45$ & 18.4 & \\
\hline L 106-15 & 140408.21 & -661437.9 & $\mathrm{~V}$ & $5 \mathrm{~s}$ & 62 & 2011.50-2015.08 & 3.58 & 8 & 16.5 & $63.47 \pm 1.96$ & $1.53 \pm 0.21$ & $65.00 \pm 1.97$ & $481.4 \pm 2.0$ & $074.1 \pm 0.40$ & 35.1 & \\
\hline SCR 1420-7516 & 142036.84 & -751605.9 & I & $6 \mathrm{~s}$ & 64 & 2009.24-2015.24 & 5.99 & 10 & 10.9 & $40.05 \pm 1.80$ & $3.07 \pm 0.27$ & $43.12 \pm 1.82$ & $142.7 \pm 1.3$ & $249.4 \pm 0.96$ & 15.7 & \\
\hline LHS 2935 & 143208.51 & +081131.3 & $\mathrm{R}$ & $7 \mathrm{~s}$ & 74 & 2000.14-2009.32 & 9.19 & 10 & 14.3 & $77.14 \pm 0.99$ & $0.24 \pm 0.02$ & $77.38 \pm 0.99$ & $471.3 \pm 0.4$ & $272.1 \pm 0.08$ & 28.9 & \\
\hline SCR 1441-7338 & 144114.42 & -733841.4 & I & $6 \mathrm{~s}$ & 62 & $2006.22-2014.44$ & 8.22 & 10 & 9.8 & $38.82 \pm 0.93$ & $1.00 \pm 0.16$ & $39.82 \pm 0.94$ & $220.7 \pm 0.7$ & $030.0 \pm 0.33$ & 26.3 & \\
\hline SCR 1444-3426 & 144406.56 & -342647.2 & $\mathrm{R}$ & $6 c$ & 75 & 2011.16-2016.20 & 5.03 & 9 & 15.3 & $65.74 \pm 1.04$ & $1.05 \pm 0.11$ & $66.79 \pm 1.05$ & $442.0 \pm 0.7$ & $190.6 \pm 0.14$ & 31.4 & \\
\hline LP $682-18$ & 151543.71 & -072521.0 & $\mathrm{~V}$ & $6 \mathrm{~s}$ & 59 & $2010.50-2015.30$ & 4.80 & 11 & 9.3 & $58.19 \pm 1.06$ & $1.01 \pm 0.33$ & $59.20 \pm 1.11$ & $294.2 \pm 0.9$ & $210.3 \pm 0.35$ & 23.6 & \\
\hline L $108-87$ & 153052.02 & $\begin{array}{llll}-68 & 01 & 17.5\end{array}$ & I & $5 \mathrm{~s}$ & 56 & 2011.50-2015.22 & 3.72 & 11 & 10.1 & $56.16 \pm 1.61$ & $2.17 \pm 0.21$ & $58.33 \pm 1.62$ & $193.3 \pm 1.7$ & $090.9 \pm 0.67$ & 15.7 & \\
\hline L 408-123AB & 154541.62 & -433029.0 & $\mathrm{R}$ & $15 \mathrm{~s}$ & 171 & 2000.58-2016.18 & 15.61 & 9 & 8.9 & $49.23 \pm 0.70$ & $1.77 \pm 0.13$ & $51.00 \pm 0.71^{\mathrm{a}}$ & $455.5 \pm 0.3$ & $216.9 \pm 0.06$ & 41.5 & $!$ \\
\hline LHS 3124 & 155121.80 & +293106.3 & $\mathrm{~V}$ & $7 \mathrm{~s}$ & 67 & 2001.43-2009.32 & 7.89 & 9 & 6.4 & $52.64 \pm 1.89$ & $0.54 \pm 0.04$ & $53.18 \pm 1.89$ & $493.9 \pm 0.7$ & $206.5 \pm 0.17$ & 44.0 & \\
\hline SCR 1601-3421 & 160155.68 & -342156.8 & I & $7 \mathrm{~s}$ & 76 & 2007.55-2016.21 & 8.66 & 10 & $\ldots$ & $48.05 \pm 0.85$ & $0.90 \pm 0.09$ & $48.95 \pm 0.85$ & $631.0 \pm 0.6$ & $118.5 \pm 0.10$ & 61.1 & \\
\hline SCR 1626-3812 & 162651.69 & -381232.6 & I & $5 \mathrm{~s}$ & 51 & 2010.20-2014.67 & 4.48 & 14 & 19.3 & $67.99 \pm 0.97$ & $1.50 \pm 0.50^{\mathrm{b}}$ & $69.49 \pm 1.09$ & $381.1 \pm 1.1$ & $228.8 \pm 0.33$ & 24.1 & \\
\hline SCR $1630-3633 \mathrm{~A}$ & 163027.23 & -363356.1 & I & $7 \mathrm{~s}$ & 71 & 2008.21-2016.20 & 7.99 & 12 & $\cdots$ & $62.96 \pm 1.30$ & $2.69 \pm 0.58$ & $65.65 \pm 1.42$ & $441.4 \pm 0.8$ & $249.0 \pm 0.20$ & 31.9 & \\
\hline SCR 1630-3633B & 163027.23 & -363356.1 & I & $7 \mathrm{~s}$ & 71 & 2008.21-2016.20 & 7.99 & 12 & $\cdots$ & $55.30 \pm 1.27$ & $2.69 \pm 0.58$ & $57.99 \pm 1.40$ & $455.5 \pm 0.8$ & $248.7 \pm 0.19$ & 37.2 & \\
\hline SCR 1636-4041 & 163657.58 & -404108.8 & $\mathrm{R}$ & $5 \mathrm{~s}$ & 53 & 2010.40-2014.45 & 4.05 & 16 & 12.2 & $44.23 \pm 1.26$ & $2.12 \pm 0.72$ & $46.35 \pm 1.45$ & $290.1 \pm 1.3$ & $193.1 \pm 0.42$ & 29.7 & \\
\hline LP 154-205 & 164755.15 & -650911.6 & I & $9 \mathrm{~s}$ & 106 & 2004.18-2015.56 & 11.38 & 10 & 9.0 & $65.73 \pm 0.97$ & $1.00 \pm 0.07$ & $66.73 \pm 0.97$ & $368.9 \pm 0.3$ & $103.9 \pm 0.08$ & 26.2 & \\
\hline G 169-29 & 165057.95 & +222705.7 & $\mathrm{R}$ & $15 \mathrm{~s}$ & 177 & $2000.57-2015.29$ & 14.72 & 8 & 16.9 & $95.06 \pm 0.83$ & $0.62 \pm 0.14$ & $95.68 \pm 0.84$ & $404.2 \pm 0.2$ & $006.3 \pm 0.04$ & 20.0 & $!$ \\
\hline SCR 1656-2046 & 165633.61 & -204637.4 & I & $4 \mathrm{c}$ & 61 & $2012.41-2015.54$ & 3.13 & 8 & 9.3 & $60.96 \pm 0.70$ & $1.33 \pm 0.15$ & $62.29 \pm 0.72$ & $271.1 \pm 0.6$ & $225.3 \pm 0.25$ & 20.6 & \\
\hline G $139-3 \mathrm{AB}$ & 165825.25 & +135810.8 & $\mathrm{R}$ & $5 \mathrm{~s}$ & 60 & 2003.53-2007.42 & 3.90 & 8 & $\cdots$ & $69.45 \pm 2.06$ & $1.45 \pm 0.11$ & $70.90 \pm 2.06$ & $400.3 \pm 1.9$ & $275.6 \pm 0.42$ & 26.8 & \\
\hline SCR 1659-6958 & 165928.14 & -695818.3 & I & $5 c$ & 63 & 2003.24-2007.55 & 4.31 & 9 & 6.4 & $47.51 \pm 1.04$ & $1.60 \pm 0.13$ & $49.11 \pm 1.05$ & $686.0 \pm 1.0$ & $213.1 \pm 0.16$ & 66.2 & $!$ \\
\hline SCR 1728-0143 & 172811.06 & -014357.0 & I & $6 \mathrm{~s}$ & 62 & 2011.50-2016.21 & 4.71 & 7 & 14.5 & $41.39 \pm 2.34$ & $3.79 \pm 0.47$ & $45.18 \pm 2.39$ & $187.2 \pm 2.2$ & $147.3 \pm 1.30$ & 19.6 & \\
\hline SCR 1731-2452 & 173103.84 & -245243.6 & I & $5 \mathrm{~s}$ & 49 & $2010.39-2014.45$ & 4.05 & 9 & 9.8 & $35.19 \pm 1.47$ & $1.50 \pm 0.50^{\mathrm{b}}$ & $36.69 \pm 1.55$ & $181.7 \pm 1.4$ & $221.1 \pm 0.85$ & 21.4 & \\
\hline L 204-148 & 173832.51 & -583234.4 & $\mathrm{R}$ & $5 \mathrm{~s}$ & 60 & 2011.50-2015.56 & 4.06 & 9 & 20.9 & $61.68 \pm 1.28$ & $1.31 \pm 0.19$ & $62.99 \pm 1.29$ & $327.9 \pm 0.9$ & $175.7 \pm 0.24$ & 24.7 & \\
\hline LTT 7077 & 174629.35 & -084236.5 & $\mathrm{~V}$ & $5 \mathrm{c}$ & 61 & $2011.50-2015.31$ & 3.81 & 10 & 19.0 & $74.48 \pm 1.52$ & $2.70 \pm 0.78$ & $77.18 \pm 1.71$ & $425.4 \pm 1.6$ & $185.8 \pm 0.33$ & 26.1 & \\
\hline SCR $1746-3214$ & 174640.66 & -321404.4 & I & $5 \mathrm{~s}$ & 60 & $2011.50-2015.31$ & 3.81 & 10 & 10.3 & $85.51 \pm 1.26$ & $1.50 \pm 0.50^{\mathrm{b}}$ & $87.01 \pm 1.36$ & $232.5 \pm 1.0$ & $062.6 \pm 0.47$ & 12.4 & \\
\hline L 415-82 & $1757 \quad 14.23$ & -415929.2 & $\mathrm{~V}$ & $4 \mathrm{c}$ & 87 & $2000.57-2003.26$ & 2.69 & 9 & 8.6 & $56.11 \pm 1.13$ & $2.21 \pm 0.13$ & $58.32 \pm 1.14$ & $372.3 \pm 1.4$ & $190.4 \pm 0.36$ & 30.3 & \\
\hline G $154-43 \mathrm{AB}$ & 180336.07 & -185850.5 & $\mathrm{R}$ & $4 \mathrm{c}$ & 66 & $2010.20-2015.29$ & 5.09 & 9 & 16.2 & $73.71 \pm 0.73$ & $3.50 \pm 0.79$ & $77.21 \pm 1.08^{\mathrm{a}}$ & $344.7 \pm 0.6$ & $154.6 \pm 0.19$ & 21.2 & $!$ \\
\hline L $43-72 \mathrm{AB}$ & $1811 \quad 15.30$ & -785922.7 & $\mathrm{~V}$ & $6 \mathrm{~s}$ & 56 & 2009.75-2015.69 & 5.95 & 9 & 13.7 & $82.72 \pm 1.58$ & $1.64 \pm 0.15$ & $84.36 \pm 1.59$ & $323.3 \pm 0.9$ & $011.1 \pm 0.28$ & 18.2 & \\
\hline LTT 7246A & $1815 \quad 12.42$ & -192406.6 & $\mathrm{~V}$ & $5 \mathrm{~s}$ & 66 & 2011.74-2015.56 & 3.82 & 9 & 15.6 & $68.30 \pm 1.30$ & $1.48 \pm 0.33$ & $69.77 \pm 1.34$ & $408.7 \pm 1.5$ & $164.6 \pm 0.36$ & 27.8 & $!$ \\
\hline SCR 1826-6542AB & 182646.83 & -654239.9 & I & $8 \mathrm{~s}$ & 89 & 2005.71-2015.30 & 9.59 & 8 & 10.3 & $65.20 \pm 0.73$ & $1.20 \pm 0.07$ & $66.40 \pm 0.73$ & $306.4 \pm 0.2$ & $178.5 \pm 0.07$ & 21.9 & \\
\hline GJ 714 & 183012.00 & $\begin{array}{lll}-58 & 1627.7\end{array}$ & $\mathrm{~V}$ & $4 \mathrm{c}$ & 63 & 2011.50-2015.42 & 3.92 & 9 & 15.5 & $67.84 \pm 2.64$ & $1.15 \pm 0.18$ & $68.99 \pm 2.65$ & $436.8 \pm 2.2$ & $182.8 \pm 0.44$ & 30.0 & \\
\hline G $141-21$ & 183619.24 & +133626.4 & $\mathrm{R}$ & $6 c$ & 85 & 2003.52-2012.58 & 9.06 & 7 & 12.2 & $82.65 \pm 1.93$ & $3.46 \pm 0.57$ & $86.11 \pm 2.01$ & $338.8 \pm 0.8$ & $034.0 \pm 0.26$ & 18.7 & \\
\hline SCR 1841-4347 & 184109.81 & -434732.8 & I & $9 \mathrm{~s}$ & 74 & 2007.55-2015.31 & 7.76 & 7 & 12.2 & $80.96 \pm 0.66$ & $1.17 \pm 0.09$ & $82.13 \pm 0.67$ & $798.7 \pm 0.3$ & $265.2 \pm 0.03$ & 46.1 & \\
\hline CE 507(B) & $1843 \quad 12.38$ & -332231.3 & V & $5 \mathrm{~s}$ & 54 & $2000.75-2011.25$ & 10.85 & 9 & $\cdots$ & $63.78 \pm 2.68$ & $2.32 \pm 0.19$ & $66.10 \pm 2.69$ & $393.8 \pm 0.7$ & $202.2 \pm 0.20$ & 28.2 & \\
\hline LTT 7419(A) & $1843 \quad 12.51$ & -332246.2 & $\mathrm{~V}$ & $5 \mathrm{~s}$ & 54 & 2000.75-2011.25 & 10.85 & 9 & 9.9 & $64.94 \pm 2.49$ & $2.32 \pm 0.19$ & $67.26 \pm 2.50$ & $389.9 \pm 0.7$ & $199.8 \pm 0.18$ & 27.5 & \\
\hline LTT 7434AB & 184557.47 & -285553.2 & $\mathrm{~V}$ & $9 \mathrm{~s}$ & 79 & 2000.58-2015.54 & 14.97 & 10 & 28.5 & $52.93 \pm 1.02$ & $2.93 \pm 0.28$ & $55.86 \pm 1.06^{\mathrm{a}}$ & $409.6 \pm 0.2$ & $135.0 \pm 0.06$ & 31.7 & $!$ \\
\hline L 345-91A & 184841.36 & -464707.8 & $\mathrm{~V}$ & $6 \mathrm{~s}$ & 37 & 2010.58-2015.42 & 4.84 & 9 & $\ldots$ & $59.52 \pm 1.85$ & $1.90 \pm 0.17$ & $61.42 \pm 1.86$ & $220.0 \pm 0.9$ & $052.6 \pm 0.48$ & 17.0 & \\
\hline L 345-91B & 184841.36 & -464707.8 & $\mathrm{~V}$ & $6 \mathrm{~s}$ & 37 & 2010.58-2015.42 & 4.84 & 9 & $\cdots$ & $54.59 \pm 1.98$ & $1.90 \pm 0.17$ & $56.49 \pm 1.99$ & $262.1 \pm 1.0$ & $054.7 \pm 0.42$ & 22.0 & \\
\hline SIP 1848-8214 & 184851.11 & -821442.0 & I & $7 \mathrm{~s}$ & 62 & 2009.63-2015.41 & 5.79 & 10 & 8.4 & $55.38 \pm 1.56$ & $1.14 \pm 0.07$ & $56.52 \pm 1.56$ & $268.7 \pm 0.7$ & $190.1 \pm 0.24$ & 22.5 & \\
\hline LP 691-15 & 184906.41 & -031517.5 & $\mathrm{R}$ & $6 \mathrm{~s}$ & 43 & 2010.50-2015.41 & 4.91 & 9 & 19.0 & $58.40 \pm 1.31$ & $1.50 \pm 0.50^{\mathrm{b}}$ & $59.90 \pm 1.40$ & $267.1 \pm 0.8$ & $092.1 \pm 0.25$ & 18.3 & \\
\hline L $489-43$ & 185225.31 & -373036.4 & $\mathrm{~V}$ & $5 c$ & 51 & $2011.50-2015.29$ & 3.80 & 8 & $\cdots$ & $64.14 \pm 1.08$ & $1.75 \pm 0.20$ & $65.89 \pm 1.10$ & $429.5 \pm 1.0$ & $100.0 \pm 0.22$ & 30.9 & $!$ \\
\hline SCR 1855-6914 & 185547.89 & -691415.1 & I & $8 \mathrm{~s}$ & 92 & 2003.51-2015.41 & 11.90 & 9 & 8.9 & $85.39 \pm 0.71$ & $1.25 \pm 0.04$ & $86.64 \pm 0.71$ & $827.7 \pm 0.2$ & $145.6 \pm 0.03$ & 45.3 & \\
\hline
\end{tabular}


Table 2

(Continued)

\begin{tabular}{|c|c|c|c|c|c|c|c|c|c|c|c|c|c|c|c|c|}
\hline Name & $\begin{array}{c}\text { R.A. } \\
\text { (hh:mm:ss) } \\
\text { (2) }\end{array}$ & $\begin{array}{c}\text { Decl. } \\
\text { (dd:mm:ss) } \\
\text { (3) }\end{array}$ & (4) & $\begin{array}{l}N_{\text {sea }} \\
(5)\end{array}$ & $\begin{array}{l}N_{\text {fr }} \\
(6)\end{array}$ & $\begin{array}{c}\text { Coverage } \\
\text { (7) }\end{array}$ & $\begin{array}{c}T \\
\text { (year) } \\
\text { (8) }\end{array}$ & $\begin{array}{l}N_{\text {ref }} \\
(9)\end{array}$ & $\begin{array}{c}\text { var } \\
(\mathrm{mmag}) \\
(10)\end{array}$ & $\begin{array}{c}\pi_{\text {rel }} \\
\text { (mas) } \\
(11)\end{array}$ & $\begin{array}{c}\pi_{\mathrm{cor}} \\
(\mathrm{mas}) \\
(12)\end{array}$ & $\begin{array}{c}\pi_{\mathrm{abs}} \\
(\mathrm{mas}) \\
(13)\end{array}$ & $\begin{array}{c}\mu \\
\left(\operatorname{mas~yr}^{-1}\right) \\
(14)\end{array}$ & $\begin{array}{l}\text { P.A. } \\
\text { (deg) } \\
(15)\end{array}$ & $\begin{array}{c}V_{\mathrm{tan}} \\
\left(\mathrm{km} \mathrm{s}^{-1}\right) \\
(16)\end{array}$ & $\begin{array}{r}\text { Notes } \\
\text { (17) }\end{array}$ \\
\hline SCR 1901-0737 & 190132.37 & -073724.3 & I & $6 \mathrm{~s}$ & 62 & 2010.66-2015.41 & 4.75 & 9 & 9.7 & $37.73 \pm 0.95$ & $1.66 \pm 0.11$ & $39.39 \pm 0.96$ & $157.7 \pm 0.6$ & $231.5 \pm 0.44$ & 19.0 & \\
\hline SCR 1901-3106 & 190159.16 & -310645.0 & I & $6 \mathrm{~s}$ & 60 & 2010.66-2015.42 & 4.76 & 9 & 9.3 & $19.06 \pm 1.17$ & $2.66 \pm 0.18$ & $21.72 \pm 1.18$ & $196.8 \pm 0.7$ & $136.6 \pm 0.41$ & 43.0 & \\
\hline LHS 5348AB & 192752.69 & $-2811 \quad 15.4$ & I & $5 \mathrm{~s}$ & 73 & 2011.50-2015.41 & 3.91 & 8 & 16.1 & $76.66 \pm 1.31$ & $1.99 \pm 0.15$ & $78.65 \pm 1.32$ & $475.0 \pm 0.9$ & $170.8 \pm 0.17$ & 28.6 & $!$ \\
\hline SCR 1931-0306 & 193104.61 & -030618.0 & I & $9 \mathrm{~s}$ & 109 & 2004.58-2013.65 & 9.08 & 8 & 17.0 & $54.89 \pm 0.55$ & $2.30 \pm 0.20$ & $57.19 \pm 0.59$ & $523.9 \pm 0.1$ & $023.4 \pm 0.03$ & 43.4 & \\
\hline L $275-26$ & 193403.94 & -522514.2 & $\mathrm{R}$ & $3 \mathrm{c}$ & 35 & 2013.39-2015.82 & 2.43 & 7 & 16.2 & $51.63 \pm 1.98$ & $0.58 \pm 0.04$ & $52.21 \pm 1.98$ & $258.1 \pm 2.5$ & $170.9 \pm 0.91$ & 23.4 & \\
\hline LHS 3492 & 195131.25 & -505537.7 & I & $5 \mathrm{~s}$ & 60 & 2007.56-2011.62 & 4.05 & 8 & 8.3 & $37.44 \pm 1.86$ & $1.65 \pm 0.13$ & $39.09 \pm 1.86$ & $817.7 \pm 1.0$ & $191.2 \pm 0.12$ & 99.2 & \\
\hline SCR 2016-7531 & 201611.25 & -753104.5 & I & $8 \mathrm{~s}$ & 81 & 2007.74-2014.44 & 6.70 & 9 & 11.6 & $65.27 \pm 1.44$ & $1.33 \pm 0.12$ & $66.60 \pm 1.44$ & $258.8 \pm 0.8$ & $079.8 \pm 0.30$ & 18.4 & \\
\hline SCR 2025-1534 & 202508.55 & -153416.1 & $\mathrm{R}$ & $4 \mathrm{~s}$ & 54 & 2011.77-2014.80 & 3.03 & 12 & 12.1 & $26.49 \pm 1.43$ & $1.06 \pm 0.14$ & $27.55 \pm 1.44$ & $173.8 \pm 1.5$ & $178.9 \pm 0.71$ & 29.9 & \\
\hline LEHPM 2-1265AB & 203301.87 & -490310.6 & $\mathrm{R}$ & $8 \mathrm{~s}$ & 106 & 2008.64-2015.54 & 6.91 & 10 & 14.1 & $59.95 \pm 0.91$ & $1.28 \pm 0.20$ & $61.23 \pm 0.93^{\mathrm{a}}$ & $241.1 \pm 0.6$ & $153.9 \pm 0.26$ & 19.1 & $!$ \\
\hline LP 567-63 & 203431.14 & -323059.9 & $\mathrm{R}$ & $7 \mathrm{~s}$ & 73 & 2008.73-2014.44 & 5.71 & 10 & 9.3 & $35.75 \pm 1.78$ & $1.44 \pm 0.13$ & $37.19 \pm 1.78$ & $204.9 \pm 1.0$ & $169.5 \pm 0.46$ & 26.1 & \\
\hline L $163-23$ & 210224.95 & -603136.1 & $\mathrm{~V}$ & $6 \mathrm{~s}$ & 60 & 2006.37-2011.42 & 5.06 & 8 & 13.4 & $30.60 \pm 1.84$ & $0.14 \pm 0.02$ & $30.74 \pm 1.84$ & $217.4 \pm 1.0$ & $136.0 \pm 0.55$ & 33.5 & \\
\hline LHS 3615 & 210321.66 & -502252.4 & $\mathrm{R}$ & $6 \mathrm{~s}$ & 86 & 2000.58-2009.55 & 8.97 & 6 & 7.7 & $38.43 \pm 1.25$ & $1.20 \pm 0.10$ & $39.63 \pm 1.25$ & $479.3 \pm 0.8$ & $142.0 \pm 0.20$ & 57.3 & $!$ \\
\hline LEPHM 2-801 & 210513.78 & -550356.3 & $\mathrm{R}$ & $6 \mathrm{~s}$ & 58 & 2008.71-2015.53 & 6.83 & 10 & 12.4 & $68.00 \pm 1.30$ & $0.96 \pm 0.06$ & $68.96 \pm 1.30$ & $327.6 \pm 0.8$ & $172.9 \pm 0.21$ & 22.5 & \\
\hline SCR 2122-4314 & 212216.92 & -431405.0 & $\mathrm{~V}$ & $5 \mathrm{~s}$ & 66 & 2008.70-2013.51 & 4.81 & 12 & 15.8 & $47.27 \pm 1.66$ & $1.50 \pm 0.23$ & $48.77 \pm 1.68$ & $223.8 \pm 1.5$ & $190.7 \pm 0.63$ & 21.8 & \\
\hline L 714-46 & 212818.29 & -221832.3 & $\mathrm{~V}$ & $4 \mathrm{c}$ & 79 & $2000.58-2003.77$ & 3.19 & 6 & 9.8 & $50.44 \pm 1.51$ & $1.39 \pm 0.10$ & $51.83 \pm 1.51$ & $314.4 \pm 1.4$ & $221.0 \pm 0.50$ & 28.8 & \\
\hline SCR 2130-7710 & 213007.00 & -771037.5 & I & $4 \mathrm{c}$ & 55 & $2011.50-2014.79$ & 3.29 & 10 & 12.1 & $46.84 \pm 1.30$ & $0.77 \pm 0.06$ & $47.61 \pm 1.30$ & $568.6 \pm 1.2$ & $117.0 \pm 0.24$ & 56.6 & \\
\hline L 2-77 & 214642.71 & -854304.6 & $\mathrm{R}$ & $5 \mathrm{~s}$ & 65 & 2011.77-2015.42 & 3.65 & 9 & 34.0 & $62.12 \pm 1.86$ & $1.28 \pm 0.21$ & $63.40 \pm 1.87$ & $297.5 \pm 1.9$ & $128.6 \pm 0.72$ & 22.2 & \\
\hline L 427-34A & 214911.35 & -413329.6 & $\mathrm{~V}$ & $6 \mathrm{~s}$ & 58 & 2010.58-2015.54 & 4.96 & 13 & $\ldots$ & $61.66 \pm 2.87$ & $0.75 \pm 0.09$ & $62.41 \pm 2.87$ & $340.7 \pm 1.4$ & $129.6 \pm 0.47$ & 25.9 & $!$ \\
\hline L 427-34B & 214911.35 & -413329.6 & $\mathrm{~V}$ & $6 \mathrm{~s}$ & 58 & 2010.58-2015.54 & 4.96 & 13 & $\ldots$ & $67.28 \pm 2.90$ & $0.75 \pm 0.09$ & $68.03 \pm 2.90$ & $337.8 \pm 1.5$ & $119.5 \pm 0.47$ & 23.5 & $!$ \\
\hline LEHPM 1-4771AB & 223009.46 & -534455.5 & $\mathrm{R}$ & $10 \mathrm{~s}$ & 101 & 2006.78-2015.83 & 9.05 & 9 & 16.2 & $63.05 \pm 1.11$ & $0.65 \pm 0.05$ & $63.70 \pm 1.11^{\mathrm{a}}$ & $743.3 \pm 0.5$ & $184.8 \pm 0.05$ & 55.6 & $!$ \\
\hline L 645-74B & 223824.67 & -292114.1 & $\mathrm{~V}$ & $7 \mathrm{~s}$ & 105 & 2000.57-2012.81 & 12.24 & 5 & 14.8 & $85.70 \pm 1.06$ & $0.53 \pm 0.06$ & $86.23 \pm 1.06$ & $260.5 \pm 0.2$ & $183.0 \pm 0.07$ & 14.3 & $!$ \\
\hline SCR 2303-4650 & 230335.61 & -465047.0 & $\mathrm{~V}$ & $7 \mathrm{~s}$ & 93 & 2009.63-2015.83 & 6.20 & 7 & 11.5 & $62.83 \pm 1.16$ & $0.57 \pm 0.07$ & $63.40 \pm 1.16$ & $192.4 \pm 0.6$ & $263.3 \pm 0.28$ & 14.4 & \\
\hline SCR 2307-8452 & 230719.67 & -845203.9 & I & $3 c$ & 75 & $2011.50-2014.80$ & 3.30 & 7 & 11.2 & $64.62 \pm 1.95$ & $1.22 \pm 0.12$ & $65.84 \pm 1.95$ & $602.9 \pm 1.9$ & $096.9 \pm 0.29$ & 43.4 & \\
\hline L 85-31 & 235325.21 & -705641.1 & I & $7 \mathrm{~s}$ & 89 & 2006.53-2014.45 & 7.92 & 7 & 8.5 & $79.44 \pm 2.49$ & $1.38 \pm 0.21$ & $80.82 \pm 2.50$ & $312.8 \pm 0.8$ & $084.9 \pm 0.24$ & 18.3 & \\
\hline LTT 9828AB & 235944.83 & -440500.1 & $\mathrm{~V}$ & $13 \mathrm{~s}$ & 129 & $2000.58-2015.83$ & 15.25 & 5 & 11.3 & $58.35 \pm 1.39$ & $0.93 \pm 0.15$ & $59.28 \pm 1.40^{\mathrm{a}}$ & $259.2 \pm 0.4$ & $352.6 \pm 0.15$ & 19.3 & $!$ \\
\hline
\end{tabular}

Notes.

${ }^{a}$ These parallax values have had the orbital motion of the system subtracted.

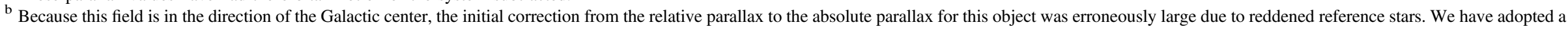
generic correction of $1.50 \pm 0.50$ mas. 
measurement, (4) the time span of the images used, (5) the parallax factor coverage (i.e., the breadth of parallax factors covered and how many frames were obtained for the "morning" and "evening" halves of the parallax ellipse for each field), (6) the DCR corrections, and (7) the correction from relative to absolute parallax.

Table 2 presents details of the astrometry observations, including the key trigonometric parallax results $(\pi)$, as well as proper motions $(\mu)$ and derived tangential velocities $\left(V_{\tan }\right)$. After the names (column 1), we list $\mathbf{J} 2000.0$ coordinates $(2,3)$, the filter in which $\pi_{\text {trig }}$ was measured (4), the number of seasons $N_{\text {sea }}$ covered $^{16}(5)$, the number of frames $N_{\text {fr }}$ acquired for each star (6), the time interval covered by the data sequence (7), the number of years of coverage (8), and the number of reference stars $N_{\text {ref }}$ used in the field (9). Results for photometric variability in the filter used for parallax observations are given in column (10), followed by the relative parallax $\pi_{\text {rel }}(11)$, the correction to absolute parallax $\pi_{\text {cor }}$ (needed because the reference stars are not at an infinite distance) (12), and final absolute parallax $\pi_{\text {abs }}$ (13), all with their associated uncertainties. The magnitude and position angle (measured east from north) of the proper motion, with their uncertainties, are given in columns $(14,15)$, followed by the derived tangential velocity $V_{\tan }(16)$, and notes (17). Objects with "!" in the notes column are discussed further in Section 4.5.

\section{RESULTS}

\subsection{Photometric Distance Estimates}

Because $\pi_{\text {trig }}$ measurements require significant resources in terms of both time and money, a careful sequence of events takes place before an object is targeted for astrometry. Generally, before placing a potentially nearby star on the program, VRI magnitudes are measured and $J H K$ magnitudes are extracted from 2MASS. Photometric distance estimates are then calculated using the relations described in Henry et al. (2004). Of the 15 possible color- $M_{K}$ relations, 12 are predictive and are calculated using the available photometry. ${ }^{17}$ These are then compared to the same relations for known single stars with accurate $\pi_{\text {trig. }}$. Any objects with distance estimates placing them closer than $20 \mathrm{pc}$ are added to the program. The distance estimates and uncertainties are provided in the final two columns of Table 1 . The few stars presented here with distance estimates placing them beyond $20 \mathrm{pc}$ are a mixture of those with distances estimated by other investigators to be nearby, relatively high proper motion stars, and stars added soon after the program started to fill in hours of R.A. that had few targets.

For six close binary systems, determination of individual parallaxes for each component was possible, as was deblending of their VRI photometry via PSF reductions; however, the 2MASS photometry is unresolved. These systems have been omitted from Figures $3-5$, as there is no clean way to include them.

Most of the stars in Table 1 were published previously in Winters et al. (2011) or Winters et al. (2015). Exceptions include 16 stars. The sample presented in Winters et al. (2015)

\footnotetext{
${ }^{16} N_{\text {sea }}$ indicates the number of seasons observed, where the six months when a star is observable counts as one season. The letter "c" indicates a continuous set of observations where multiple nights of data were taken in each season, whereas an "s" indicates scattered observations when some seasons have only a single night of observations.

17 Only two of the stars offered here have fewer than 12 relations: GJ 714 has 10 relations and LP $349-25 \mathrm{AB}$ has 11 relations.
}

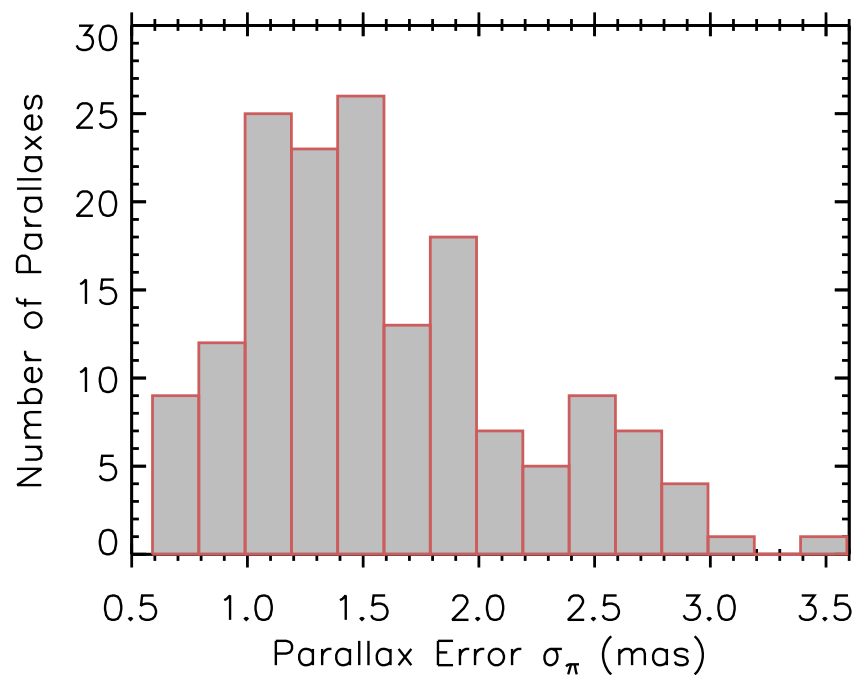

Figure 2. A histogram of the $\pi_{\text {trig }}$ uncertainties for the 161 parallaxes presented here, illustrating that most errors are less than 2 mas, with a mean parallax error for the entire sample of 1.6 mas.

were southern $\mathrm{M}$ dwarf primaries; thus, neither the eight northern $\mathrm{M}$ dwarfs nor the seven secondary components presented here were included in that publication. One additional southern M dwarf primary (SCR 2303-4650) was overlooked in previous publications and is offered here for the first time.

We note that the distance estimates reported here may be slightly different from those reported previously, due to the acquisition of more VRI photometry that improves the estimate. These new results thus supersede previous results.

\subsection{Trigonometric Distance Measurements}

The astrometry results presented in Table 2 represent the largest set of $\pi_{\text {trig }}$ measurements with uncertainties less than $\sim 3$ mas (milliarcseconds) measured for stars in the southern hemisphere since YPC and HIP. The distribution of the $\pi_{\text {trig }}$ uncertainties in Figure 2 illustrates that most of the measurements have errors less than 2 mas, with a mean value of 1.6 mas. A large $\pi_{\text {trig }}$ error is usually caused by a sparse or asymmetric reference field or the presence of a close (gravitationally bound or background) star. We note that targets in the direction of the Galactic center will have reddened reference stars that corrupt the correction from relative to absolute parallax because their distance estimates are too close. For these objects, we have adopted generic corrections of $1.50 \pm 0.50$ mas, as noted in Table 2 .

Comparisons of the photometric distance estimates to the accurate trigonometric distances provide valuable information about the targets. Unresolved binaries will have underestimated photometric distances, due to the added light from unseen companion(s). Unresolved equal-magnitude binaries will have distance estimates underestimated by a factor of sqrt(2). Light from fainter companions will decrease this offset, whereas light from additional unresolved companions will increase this offset. Low-mass subdwarfs will have overestimated distances, as they are underluminous at a given color; however, no known subdwarfs are included in the sample presented here.

Figure 3 compares the photometric distance estimates to the measured trigonometric distances, with the solid line tracing distances that match. A group of points with larger 


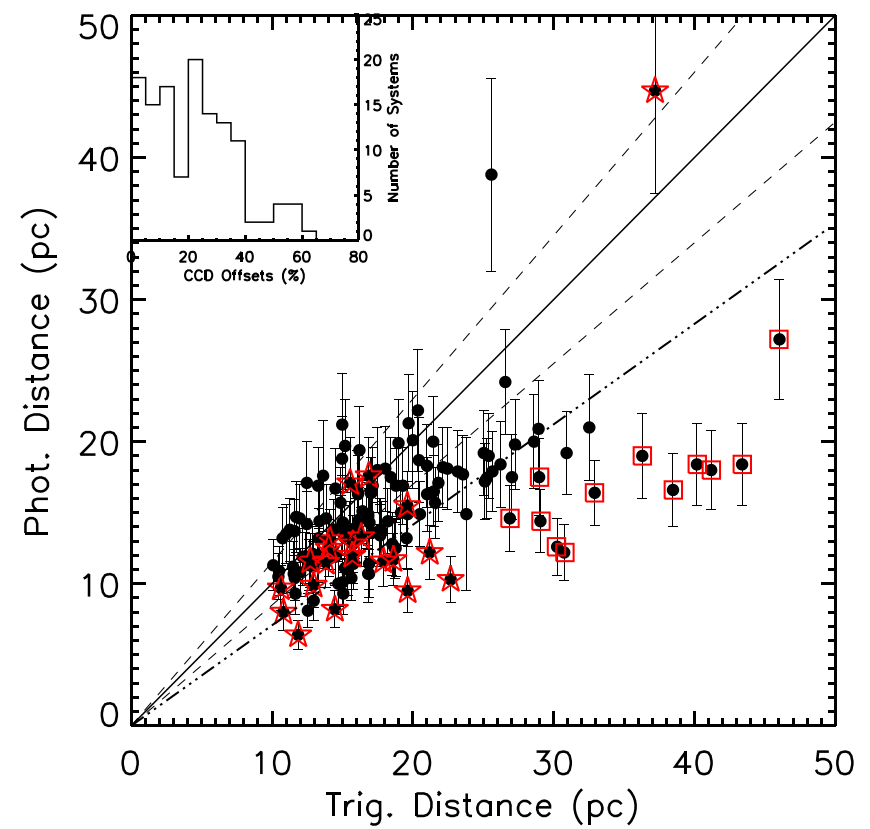

Figure 3. A comparison of distance estimates from VRIJHK photometry vs. distances measured using $\pi_{\text {trig }}$ for almost the entire sample of stars presented here. The six multiple systems with joint 2MASS photometry that prevents the calculation of individual photometric distance estimates (SCR 0644-4223AB, LP 382-56AB, SCR 1630-3633AB, LTT 7246AB, L 345 $91 \mathrm{AB}, \mathrm{L} 427-34 \mathrm{AB})$ are not included in this plot, nor are the two stars with distances $>50 \mathrm{pc}$ (CE 440-87 and WT 1928). Uncertainties in the trigonometric distances are smaller than the symbols. Known unresolved multiples with blended photometry are enclosed by open red stars. Candidate unresolved multiples are enclosed with open red squares. The diagonal solid line represents 1:1 correspondence in distances, while the dashed lines indicate the $15 \%$ uncertainties associated with the CCD distance estimates. The dash-dot line traces the location where the trigonometric distance exceeds the photometric estimate by a factor of sqrt(2), corresponding to an equal-luminosity pair of stars. The inset histogram indicates the distribution of the distance offsets between the photometric and trigonometric distances for the single stars. For these single stars, the absolute mean offset is $21 \%$, slightly higher than the $15 \%$ systematic error determined by Henry et al. (2004).

trigonometric distances than photometric estimates is evident, primarily due to a number of both confirmed and candidate unresolved binaries. The dash-dot line indicates the boundary at which the trigonometric distance is larger than sqrt(2) times that of the photometric distance, corresponding to an equalluminosity pair of unresolved stars. Points for candidate binaries below this line, taking into account uncertainties in their photometric distances, are enclosed with open red squares, whereas points for known unresolved multiples are enclosed with red stars. Some of the stars below the dash-dot line may be young stars that are overluminous because they have inflated radii, resulting in underestimated photometric distance estimates. However, we expect the number of unidentified young stars in this sample to be small because we have vetted the entire observing list for young stars with various attributes of youth, e.g., X-ray emission and space motion that matches known young moving groups. The inset histogram shows the distribution of the absolute deviations between the two distances. For the presumed single stars within $25 \mathrm{pc}$, the mean absolute deviation is $21 \%$, a bit higher than the $15 \%$ reported in Henry et al. (2004), the offset outlined with dashed lines. This is due to the presence of the candidate binaries mentioned above. Both the known and candidate multiples are discussed in Section 5.

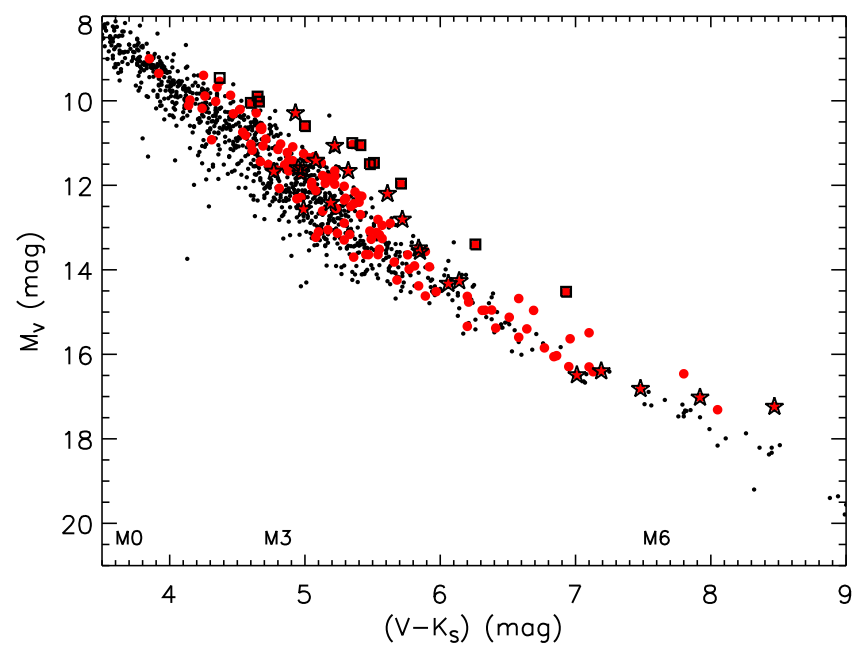

Figure 4. An observational $\mathrm{HR}$ diagram for the $\mathrm{M}$ dwarf systems presented here, with $M_{V}$ and $(V-K)$ used as proxies for luminosity and temperature, respectively. Known multiples with blended photometry are enclosed in open stars. Candidate binaries are elevated above the main sequence or have distance mismatches and are enclosed in open squares. The small background points are presumed single stars from the RECONS 25 PC Database. Spectral type estimates are given along the bottom; however, M9V corresponds to a $(V-K)$ color of 9.5 and is thus off the plot. Not included on this plot are the six close multiple systems with joint 2MASS photometry.

\subsection{Stars on the HR Diagram}

Figure 4 is an observational HR diagram illustrating the stars presented here, using $M_{V}$ for luminosity and $V-K$ for temperature. The small black points are single stars from the RECONS 25 Parsec Database (see Section 7) for reference, while the stars with new trigonometric distances are shown as larger red points. Multiples, both known (stars) and candidates (squares), are indicated and described in Section 5. The candidates were identified based on their positions above the main sequence on this plot and from mismatches between their photometric distance estimates and their trigonometric distances shown in Figure 3. As described in Section 5.4, these objects are overluminous for their distances and we thus expect them to be either near-equal luminosity and mass binaries, or young. The new multiples described in Sections 5.1 and 5.2 whose positions on this diagram are within the main sequence are expected to have unseen companions that contribute little light to the systems. Two of the new systems whose positions are slightly below the main sequence (SCR 0723-8015AB and LTT 9828AB) may have white dwarf companions, as the magnitudes of their perturbations are quite large, and yet they are not elevated above the main sequence, nor is there a mismatch between their photometric and trigonometric distances.

\subsection{Stellar Variability}

We also investigate the photometric variability of stars observed during the program using the astrometry frames to reveal short- or long-term photometric changes, such as flares or stellar cycles. Results are given in column (10) of Table 2. In short, the instrumental magnitudes of the parallax stars are compared to the ensemble of reference stars, with corrections made for varying integration times, seeing differences, etc., between frames in a series. Reference stars exhibiting variability are removed from the analysis. Details on the 


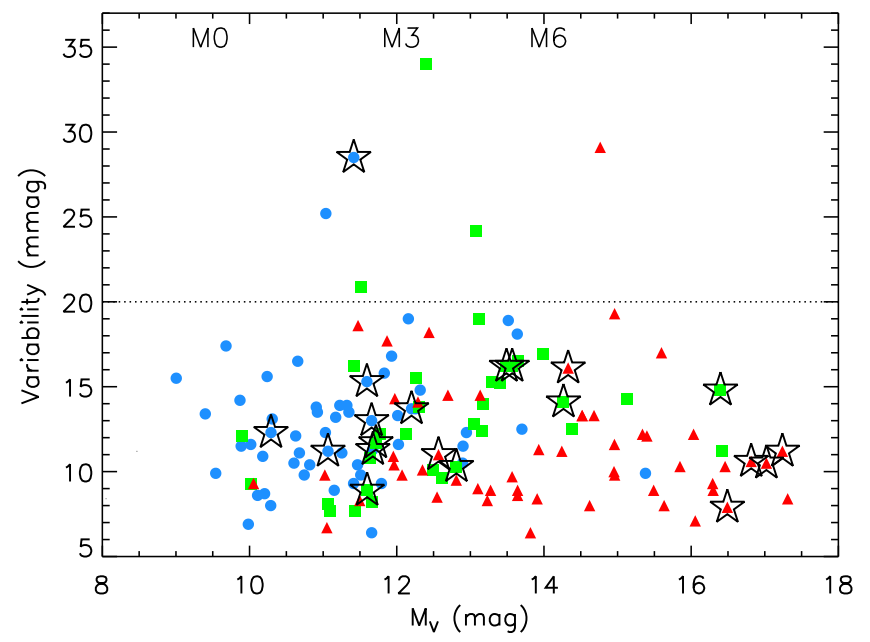

Figure 5. Variability of the stars (in $V R I$ ) as a function of $M_{V}$. Stars observed in the $V$-band are indicated as blue points, in the $R$-band as green squares, and in the $I$-band as red triangles. Close binaries with blended photometry are noted with open black stars. The dotted line marks the 20 mmag (2\%) variability boundary between active (above) and inactive (below) stars. Spectral type estimates are indicated along the top of the plot; however, M9V corresponds to an $M_{V}$ magnitude of 20.0 and is thus off the plot.

methodology can be found in Jao et al. (2011) and Hosey et al. (2015).

We find that most of the stars presented here vary by less than the 20 mmag (2\%) level we defined in Hosey et al. (2015) to be the threshold for significantly variable stars. This threshold is based on our measurement limit of $\sim 7 \mathrm{mmag}$, which we adopt as the $1-\sigma$ minimum deviation threshold for the carefully focused images required for the astrometry program. ${ }^{18}$ The limit can, and has been, reduced to $2-3 \mathrm{mmag}$ on the $0.9 \mathrm{~m}$ using defocused images and longer integrations. The 15 stars without variability measurements in Table 2 (a) are close multiples separated by a few arcseconds that are photometrically entangled, (b) were corrupted by a background star during many images in the series, or (c) were underexposed relative to the primary star so that the variability measurement was deemed unreliable.

Six stars were variable by more than $20 \mathrm{mmag}$, with L 2-77 exhibiting the largest value at $34.0 \mathrm{mmag}$. The other variable stars are LTT 7434AB (28.5 mmag), SCR 1214-4603 (27.3 mmag), L 532-12 (25.2 mmag), LEHPM 1-1343 (24.2 mmag), and L 204-148 (20.9 mmag). Figure 5 illustrates variability of the targets in their respective parallax filters (VRI) as a function of their $M_{V}$. Unexpectedly, we do not see any noticeable increase in variability in the bluest, $V$, filter. We note that three of the six variable stars (above the $20 \mathrm{mmag}$ line) were observed in the $R$-band, but based on the small number of variable stars, we do not feel that any trend is evident. We do not see any relation between the presence of an unresolved stellar companion and the magnitude of the variability, although our astrometric series is not sensitive to very close systems with orbital periods of a few days in which tidal effects may cause stellar activity. Overall, the results for the 145 stars presented here agree with the results presented in Hosey et al. (2015), with an overlap of 44 stars between the two samples.

We highlight four of the most interesting variable objects here. Figure 6 highlights two of the most variable stars in the

\footnotetext{
${ }^{18}$ Four stars have variability measured to be slightly less than $7 \mathrm{mmag}$, at 6.4-6.9 mmag.
}

sample. In the left panel, SCR 1214-4603 shows evidence of a flare event in 2011 with a temporal coverage of eight years. In the right panel is shown L 2-77 with almost four years of coverage. This star was fairly stable through 2012, but has recently started showing signs of flares and spots.

Shown in the left panel of Figure 7 is the clear stellar cycle of SCR 0702-6102, with a period on the order of a decade. The right panel shows the long-term increasing brightness of $G$ 169-29, originally presented in Hosey et al. (2015). Five additional years of data are available for analysis, confirming the brightening trend that now extends to 15 years. Note that the variability levels for these stars-9.8 mmag for SCR 07026102 and 17.8 mmag for G 169-29—-do not meet our threshold for significant variability, although it is clear that both show long-term changes in brightness. As we accumulate more data for hundreds of stars, we will explore additional facets of the long-term variability of red dwarfs that occur below $20 \mathrm{mmag}$.

\subsection{Notes on Individual Systems}

Here we present details for some of the more interesting or complicated systems, ordered by R.A. The first four digits of both the R.A. and decl. are provided in parentheses after the names, and figure numbers are listed if plots of the astrometry series are included.

LP 349-25AB $(0027+2219)$, Figure 8: This star shows a perturbation in both axes. With over 10 years of data, the orbit appears to have recently wrapped in our astrometry observations. The system is overluminous, resulting in a photometric distance estimate of $8.2 \mathrm{pc}$, compared to its trigonometric distance of $15.4 \mathrm{pc}$, implying that the companion contributes significant flux to the system in the I-band. We note that Forveille et al. (2005) report a companion detected via AO in 2004 with $\rho=0$ ". 107 and $\theta=7^{\circ}$, with $\Delta H=0.4 \mathrm{mag}$.

LTT 313 (0035-1004): The trigonometric distance for this star is about twice its photometric distance $(30.0 \pm 1.2 \mathrm{pc}$ versus $17.5 \pm 2.7 \mathrm{pc})$. We detect a hint of a perturbation in the decl. axis (not shown), but at a distance $>25 \mathrm{pc}$, we are no longer following this star to confirm or refute the possible perturbation.

L 294-92AB (0147-4836), Figure 8: This star shows a perturbation in both axes, although both types of distances match within the uncertainties: $11.5 \pm 1.8 \mathrm{pc}$ for the photometric distance versus $13.8 \pm 0.3 \mathrm{pc}$ for the trigonometric distance. Thus, the companion is likely of low mass and contributes little light to the system in the $R$-band. The orbit has not yet wrapped in our astrometric series.

$L$ 88-43 (0153-6653): The large uncertainty on the $\pi_{\text {trig }}$ for this object is due to its weak reference field and short integrations, typically only $15-30 \mathrm{~s}$ in $R$.

LP 831-45AB (0314-2309), Figure 8: This system exhibits a perturbation, very obvious in decl., resulting in a $\sim 6$ year period for the pair. The distances are $12.8 \pm 2.0 \mathrm{pc}$ for the photometric distance versus $15.7 \pm 0.3 \mathrm{pc}$ for the trigonometric distance, implying that this is an unequal mass binary.

$L$ 591-42AB (0436-2721): While one component lies within $25 \mathrm{pc}$ (A has $\pi=42.02 \pm 2.48$ mas), and the other beyond (B has $\pi=38.98 \pm 2.59$ mas), these results agree within the somewhat large uncertainties. The weighted mean $\pi_{\text {trig }}$ is $40.57 \pm 1.79$ mas, placing it just within $25 \mathrm{pc}$. In 2001, the two stars were separated by $\sim 50^{\prime \prime}$ at a position angle of $\theta=196^{\circ}$ (Jao et al. 2003). 

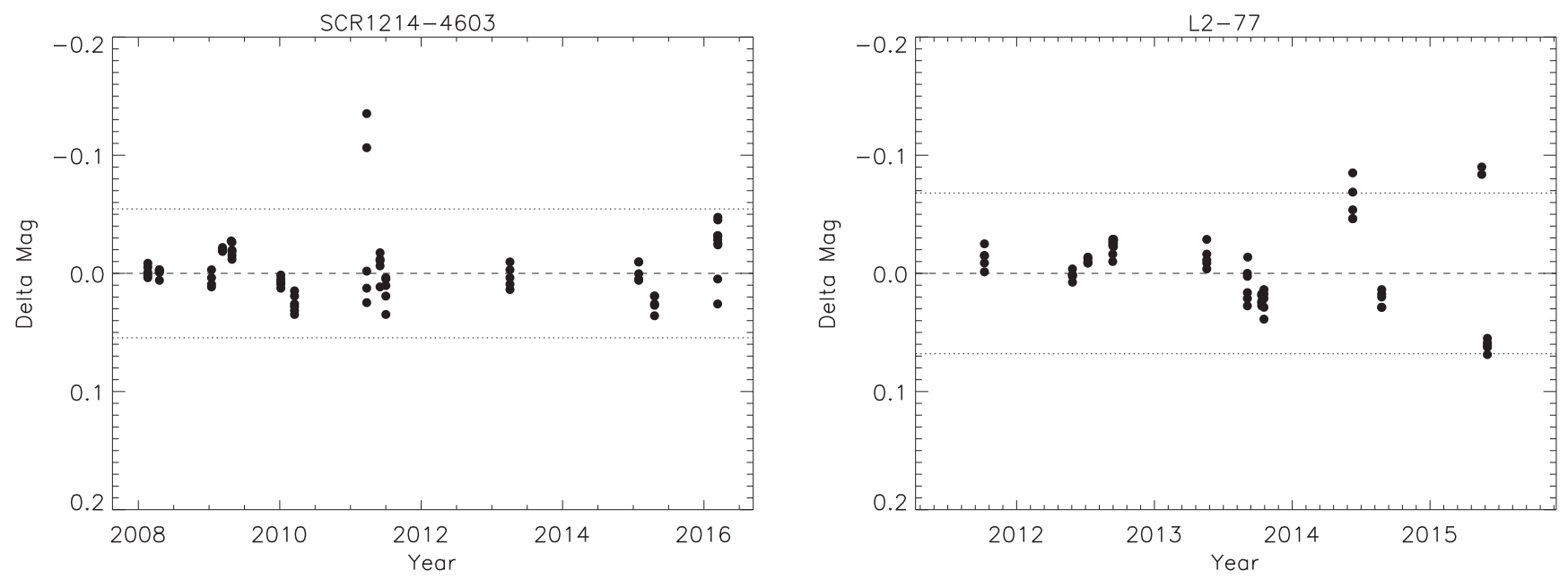

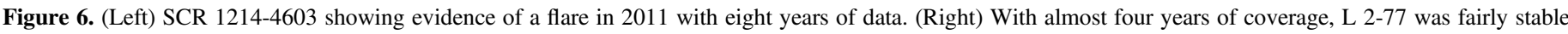

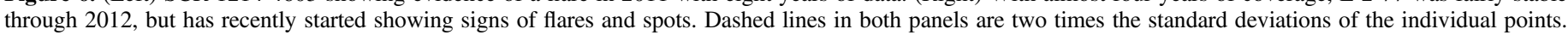

SCR 0702-6102 (0702-6102), Figure 7: The discrepant distances for this star $(10.7 \pm 2.0 \mathrm{pc}$ for its photometric distance versus $16.8 \pm 0.4 \mathrm{pc}$ for its trigonometric distance) and its elevation above the main sequence in the HR diagram in Figure 4 suggest that this is an unresolved binary or a young star. The astrometric data show a hint of a perturbation with an amplitude $\sim 20$ mas on a relatively short timescale, but the period is highly uncertain. We see evidence of a stellar cycle with a period of about a decade in the 11 years of data in-hand, as shown in the left panel of Figure 7.

SCR 0723-8015AB (0723-8015), Figure 8: This star exhibits a large perturbation in both axes, but the orbit has not yet wrapped, even after 13 years of data. The star has a trigonometric distance $(15.9 \pm 0.7 \mathrm{pc})$ that agrees with its photometric distance $(17.1 \pm 3.2 \mathrm{pc})$, suggesting that the companion may be a white dwarf that contributes minimal light at $I$.

SCR 0838-5855AB (0838-5855), Figure 8: This star was initially thought to be within $10 \mathrm{pc}$, with a photometric distance of $8.0 \pm 1.3 \mathrm{pc}$; however, its trigonometric distance of $10.6 \pm 0.1 \mathrm{pc}$ places it just beyond the $10 \mathrm{pc}$ horizon. This star shows evidence of a perturbation in both axes in a data set spanning 10 years, with an amplitude in R.A. that is, so far, larger than that in decl.

LP 788-1AB (0931-1717), Figure 8: This system exhibits a perturbation that is evident in data acquired regularly since 2011 December. The distances agree within the uncertainties: $12.5 \pm 2 \mathrm{pc}$ for its photometric distance, compared to $14.7 \pm 0.3 \mathrm{pc}$ for its trigonometric distance, implying a companion that emits little flux at $I$.

LP 848-50AB (1042-2416), Figure 8: This object was initially thought to be within $10 \mathrm{pc}$, based on its photometric distance of $9.7 \pm 1.6 \mathrm{pc}$, but the astrometric analysis shows it to be a close binary, with a trigonometric distance of $11.3 \pm 0.3 \mathrm{pc}$. The orbit shows no signs of wrapping in the current data set that spans six years. It is possible that the companion is a white dwarf, given the large amplitude of the perturbation in the $R$-band-note the 100 mas scale in the panels of Figure 8(b) - and reasonable agreement of the two distances.

LTT 4004AB (1054-0718): We find a new close companion, $\mathrm{B}$, indicated by elongated images but no resolution of the two stars down to 1 !" 0 . There may be more than two stars present, given that the trigonometric distance of $21.2 \mathrm{pc}$ is well over 1.4 times that of the photometric distance of $12.2 \mathrm{pc}$. The blinking of SuperCOSMOS images using Aladin reveals no other nearby stars that could be background sources at the epochs of the astrometry images. Alternatively, this may be a young stellar binary.

LP 908-10 (1203-2923): This object moved in front of a background source that corrupted both the astrometry and variability analyses. Frames taken in 2003-2013 were not used in the reduction, and we do not report the variability here.

SCR 1230-3411AB (1230-3411), Figures 8 and 9: A perturbation is evident in both the R.A. and decl. axes with a period of $\sim 5$ years. The trigonometric distance is $18.7 \pm 0.6 \mathrm{pc}$ versus the photometric distance of $11.7 \pm 1.8 \mathrm{pc}$, suggesting that the two stars have similar luminosities in the $R$-band. The luminosities are not identical, however, or we would see no perturbation of the photocenter.

L 327-121AB (1233-4826), Figure 8: A long-term perturbation is seen spanning the full five years of the current data set. The photometric distance estimate is $10.3 \pm 1.6 \mathrm{pc}$ compared to the trigonometric distance of $22.7 \pm 0.7 \mathrm{pc}$, indicating that the unseen companion contributes significant flux at $V$. In fact, there may be more than one companion to cause such discordant distances, or the system is young and overluminous.

WT 1962AB (1259-0730), Figure 8: We detect a long-term astrometric perturbation, likely caused by a low mass companion because minimal light is contributed by the unseen secondary: the photometric distance is $44.7 \pm 7.2 \mathrm{pc}$ and the trigonometric distance is consistent at $35.2 \pm 1.7 \mathrm{pc}$.

$L$ 408-123AB (1545-4330), Figures 8 and 9: The photometric distance of $15.5 \pm 2.4 \mathrm{pc}$ is closer than the trigonometric distance of $19.2 \pm 0.4 \mathrm{pc}$. A clear perturbation is seen in both axes in frames taken at $R$ with an orbit that has wrapped, indicating a period of $\sim 9$ years.

G 169-29 $(1650+2226)$, Figure 7: As illustrated in the second panel of Figure 7, and as first reported in Hosey et al. (2015), this object shows a remarkable increase in brightness of $\sim 60$ mmag $(6 \%)$ that now stretches to 15 years in $R$.

$G$ 154-043AB (1803-1858), Figure 8: This system shows a perturbation in the decl. axis only that is longer than our current coverage of five years. The estimated photometric distance is $9.9 \pm 1.6 \mathrm{pc}$, whereas the trigonometric distance is 

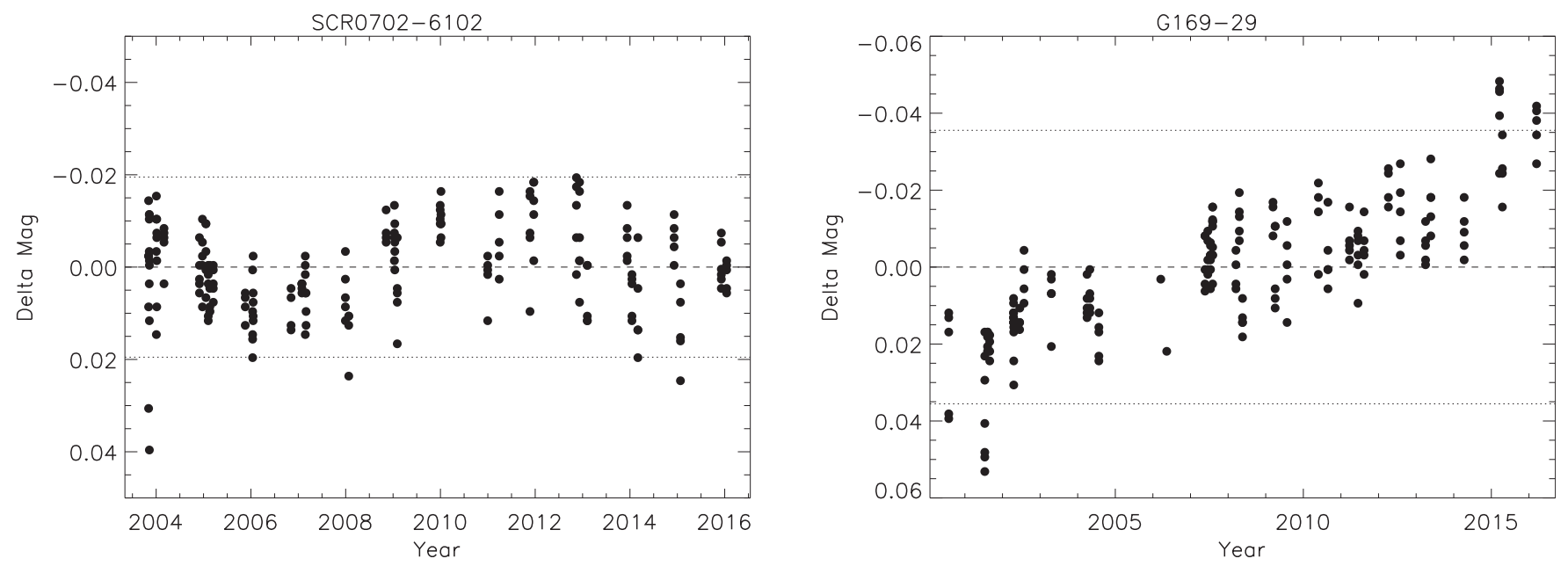

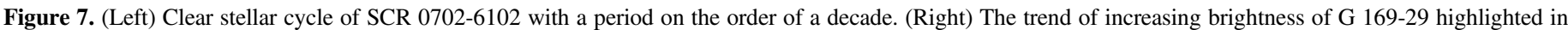
Hosey et al. (2015) now extends to 15 years. Dashed lines in both panels are two times the standard deviations of the individual points.

$13.0 \pm 0.2 \mathrm{pc}$, implying significant flux from the unseen companion at $R$.

LTT 7246AB (1815-1924): We find a new companion, B, at a separation of $3 !^{\prime \prime} 43$ at $\theta=218^{\circ} .2$ in 2011 , and at $3 . \prime 53$ at

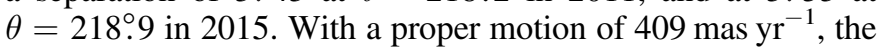
source is physical. The $\pi_{\text {trig }}$ reported is only for the A component, as the secondary is faint. The two stars have $\Delta V=2.2 \mathrm{mag}, \Delta R=2.0 \mathrm{mag}$, and $\Delta I=1.5 \mathrm{mag}$.

LTT 7434AB (1845-2855), Figure 8: This target shows a perturbation, but the orbit does not yet appear to have wrapped in our data, even with 15 years of astrometric coverage (there is a gap in the data sequence from 2004-2010). Its photometric distance is 1.4 times nearer than its trigonometric distance $(11.6 \pm 1.8 \mathrm{pc}$ versus $16.4 \pm 0.4 \mathrm{pc})$, implying equal luminosity components, but because we see the perturbation, the components must have different luminosities, or there are more than two stars in the system. We note that Bonfils et al. (2013) report this as an SB2.

L 489-43 (1852-3730): A contaminating background source corrupts the variability analysis of this star. This source may also have corrupted the VRI photometry, as the photometric distance is underestimated at $11.1 \pm 1.9 \mathrm{pc}$, compared to its trigonometric distance of $15.7 \pm 0.3 \mathrm{pc}$. However, the source does not affect the astrometry, which shows residuals to the parallax and proper motion fits of less than 2 mas on both the R.A. and decl. axes. Given that no unseen companion is evident in the astrometry, the photometric distance estimate likely suffers from additional flux from the background source.

LHS 5348AB (1927-2811): In frames with exceptional seeing, this star is obviously elongated due to a companion found at $\sim 1^{\prime \prime}$. The system's trigonometric distance is $12.7 \pm 0.2 \mathrm{pc}$, compared to its photometric distance of $11.6 \pm 1.9 \mathrm{pc}$, indicating that the companion contributes very little light to the system at $I$.

LEHPM 2-1265AB (2033-4903), Figure 8: This system shows a perturbation with a period of $\sim 5$ years, seen only in the decl. axis. The system's photometric and trigonometric distances are $13.3 \pm 2.1 \mathrm{pc}$ and $16.7 \pm 0.4 \mathrm{pc}$, respectively, so the secondary contributes only a modest amount of light, if any, to the system at $R$.

$L 427-34 A B(2149-4133)$ : This is a binary with a projected separation of $\sim 3^{\prime \prime}$, making reductions challenging. We measure $\pi_{\text {trig }}$ for both components that are consistent within the relatively large uncertainties of $\sim 3$ mas. We do not report variability results for this system, as the small separation corrupts the flux measurements, but we do estimate the $\Delta V$ $\sim 0.3 \mathrm{mag}$. The photometric distance for the unresolved pair is $11.4 \pm 2.0 \mathrm{pc}$, compared to the trigonometric distance of $\mathrm{A}$ of $16.0 \pm 0.8 \mathrm{pc}$ and the trigonometric distance of $\mathrm{B}$ of $14.7 \pm 0.7 \mathrm{pc}$.

LEHPM 1-4771AB (2230-5344), Figures 8 and 9: A large perturbation is evident in R.A., but is muted in decl. A comparison of the photometric distance $(12 \pm 1.8 \mathrm{pc})$ and the trigonometric distance $(15 \pm 0.3 \mathrm{pc})$ indicates that the companion likely contributes only a modest amount of flux to the system in $R$.

$L$ 645-74B (2238-2921): We present $\pi_{\text {trig }}$ for only the B component of this system, as the A component is saturated in most of the images. However, we know the A component to also be an $\mathrm{M}$ dwarf, based on its $(V-K)$ color of $4.4 \mathrm{mag}$ (Winters et al. 2015), so we include this system as a nearby $M$ dwarf pair.

SCR 2303-4650 (2303-4650): There may be an unseen companion orbiting with a period of less than two years. The typical cadence of our observations is not conducive to such an orbital period, so monthly observations will be required to confirm or refute the possible companion. For now, we note this object as a candidate binary.

LTT 9828AB (2359-4405), Figures 8 and 9: This system shows a clear perturbation, resulting in a $\sim 11$ year orbit for the pair that has wrapped in our data. Its photometric distance of $17.6 \pm 2.7 \mathrm{pc}$ and its trigonometric distance of $15.7 \pm 0.6 \mathrm{pc}$ agree within the uncertainties, so the companion contributes very little flux at $V$.

\section{MULTIPLE SYSTEMS}

\subsection{New Multiples-16 Unseen Companions}

In addition to distance measurements, long-term astrometric monitoring also sometimes reveals the presence of unseen companions $^{19}$ at small separations $\left(<1^{\prime \prime}\right)$ from the star being

\footnotetext{
${ }^{19}$ We note that component refers to any member of a multiple system. The primary is either a single star or the most massive (or brightest in the $V$-band) component in the system, and companion refers to a member of a multiple system that is less massive (or fainter, again in the $V$-band) than the primary star.
} 

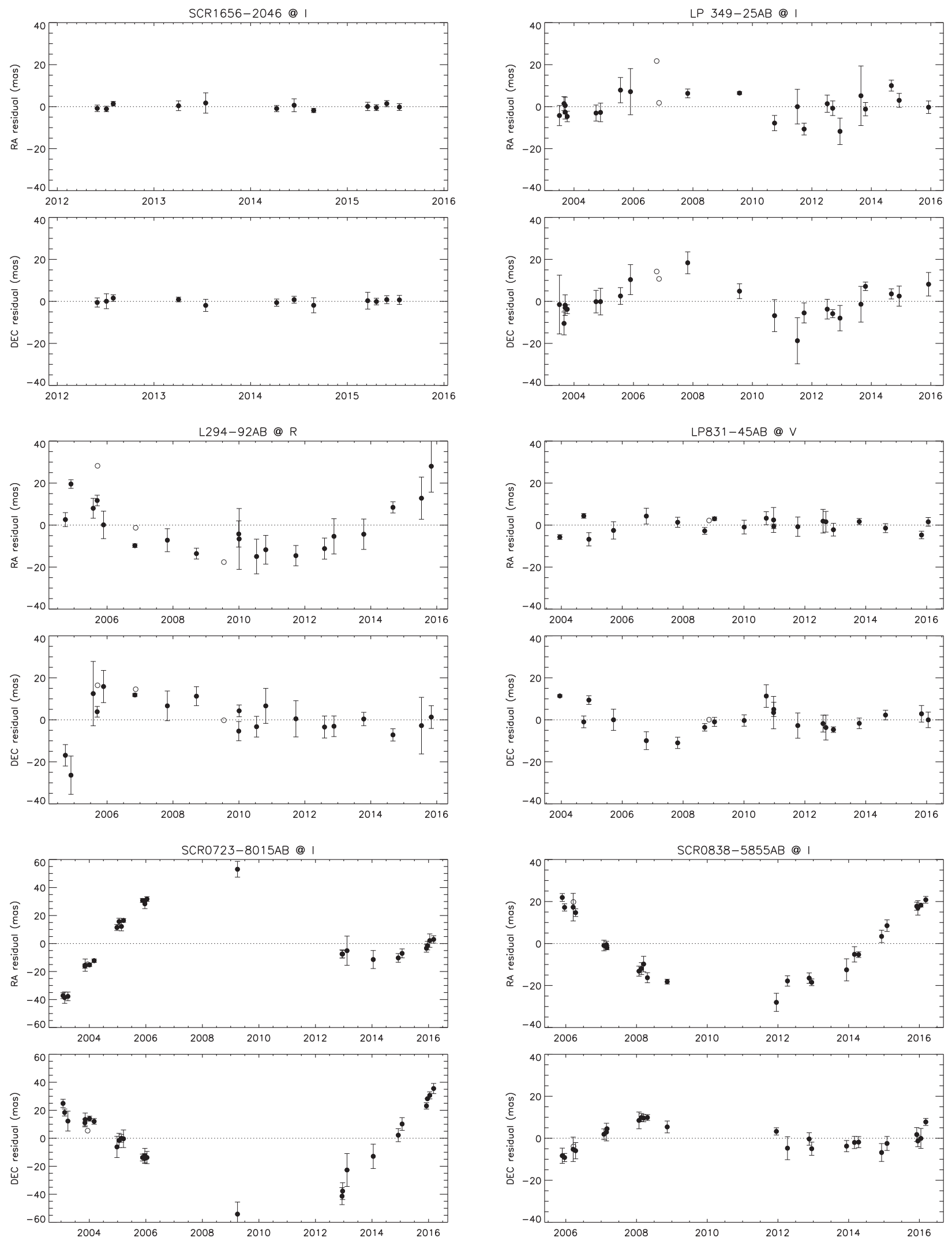

Figure 8. Nightly mean astrometric residual plots in R.A. and decl. for SCR 1656-2046, LP 349-25AB, L 294-92AB, LP 831-45AB, SCR 0723-8015AB, SCR 08385855AB, LP 788-1 AB, LP 848-50AB, SCR 1230-3411AB, L 327-121AB, WT 1962AB, L 408-123AB, G 154-43AB, LTT 7434AB, LEHPM 2-1265AB, LEHPM 1$4771 \mathrm{AB}$, and LTT $9828 \mathrm{AB}$. The astrometric signatures of each system's proper motion and parallax have been removed. SCR 1656-2046 is shown as an example of a single (i.e., non-perturbed) star with residuals clustered closely around zero. 

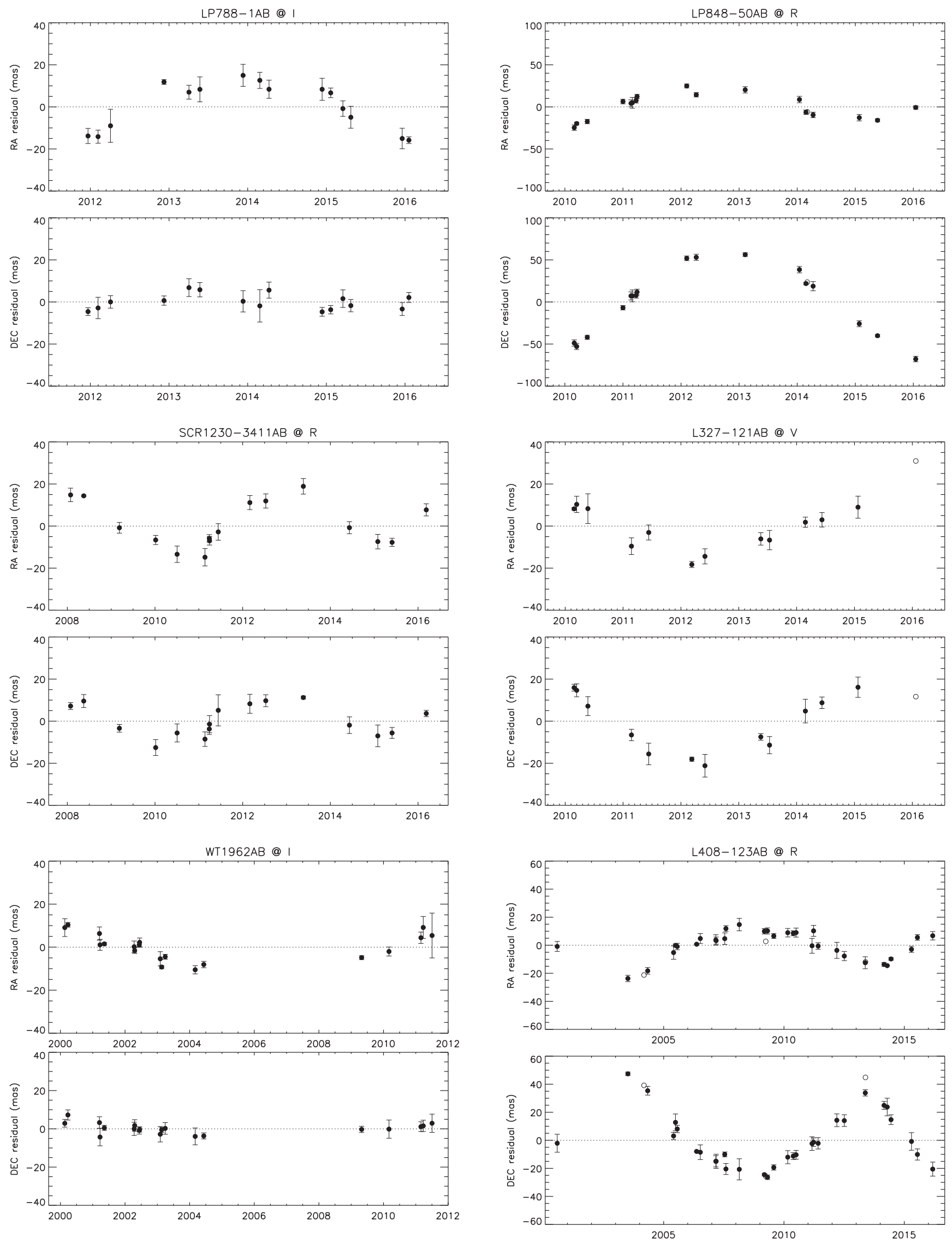

Figure 8. (Continued.) 

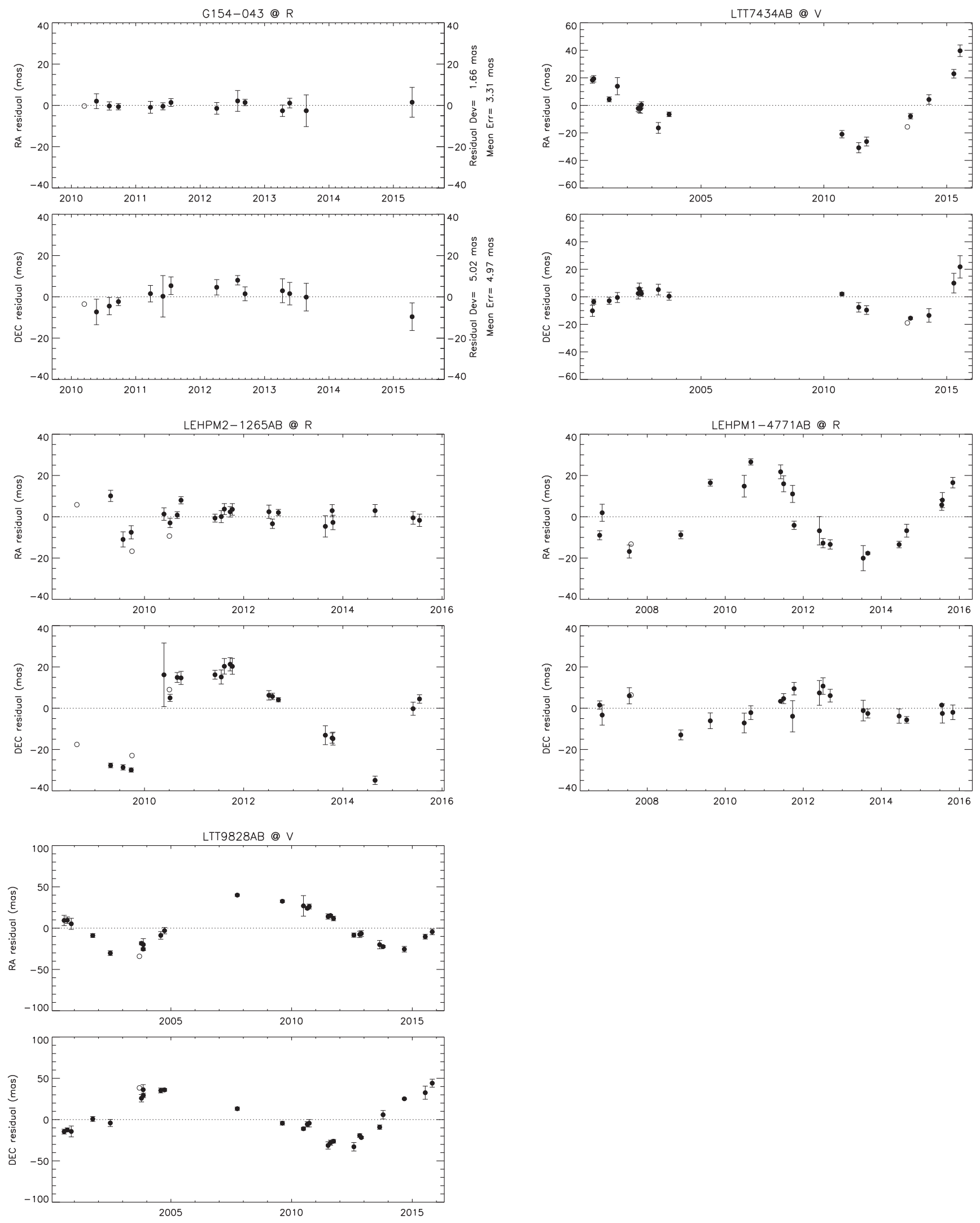

Figure 8. (Continued.)

investigated. Previous publications in this series (see references in Section 1), as well as Bartlett et al. (2009), have presented results on a few of these new binaries, which are valuable for mass determinations due to their short orbital periods. Of the systems presented here, 16 were found to exhibit astrometric perturbations indicative of unseen companions. The arcs of the 

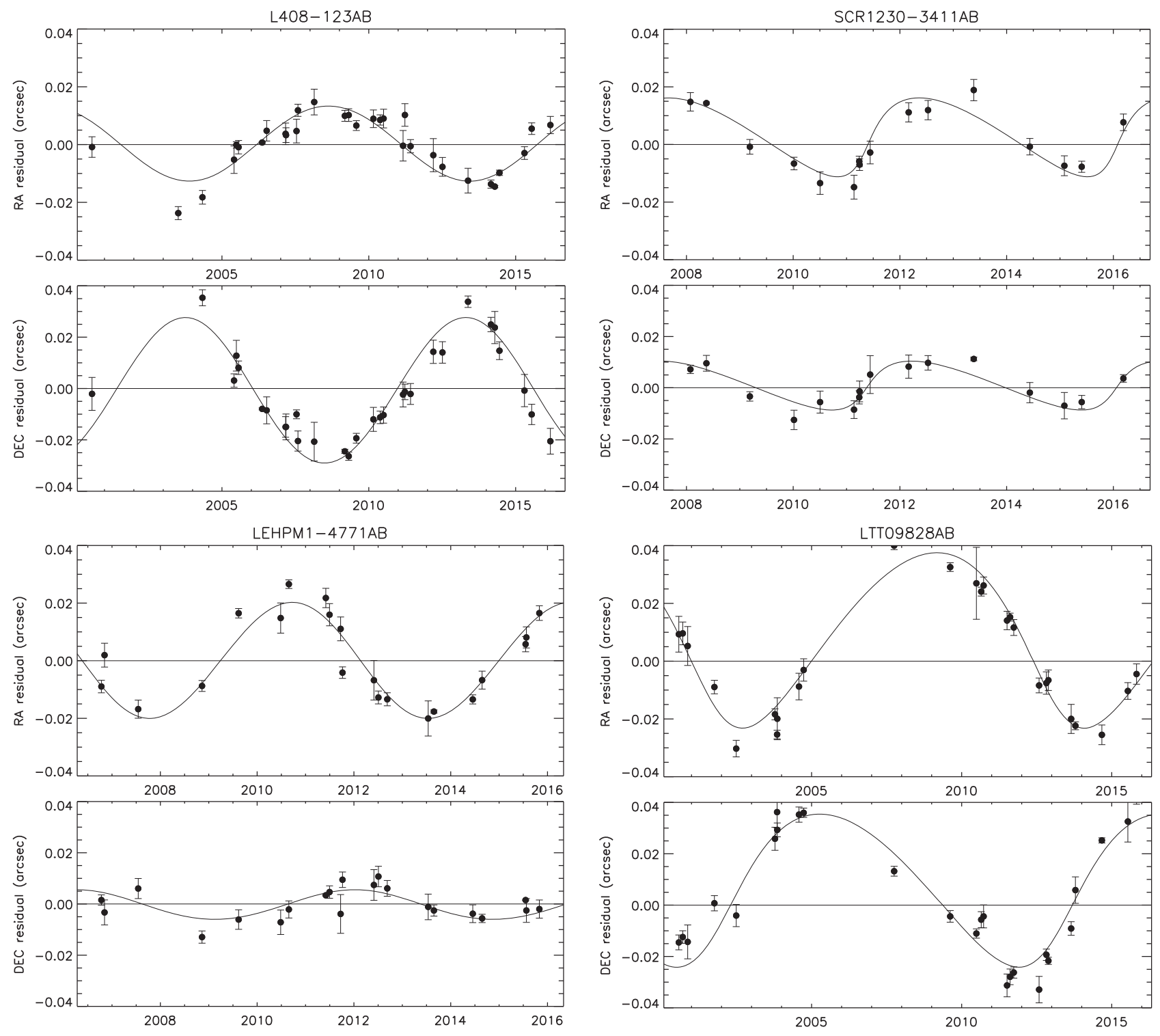

Figure 9. Orbital fits for four new binaries with complete orbits. Orbital elements are given in Table 3.

orbits were fit and removed, the target positions adjusted, and a new solution for the parallax and proper motion was obtained. These perturbations, illustrated in Figure 8, represent changes in the positions of the photocenters of the target stars evident after the parallax and proper motions have been removed. The $\pi_{\text {trig }}$ values given in Table 2 for these 16 systems represent values that include adjustments for orbital motion and are noted.

From van de Kamp (1975), the magnitude of the perturbation in the photocenter, $\alpha$, follows the relation $\alpha=(B-\beta) a$, where $\mathrm{B}$ is the fractional mass $M_{B} /\left(M_{A}+M_{B}\right)$, the relative flux $\beta$ is expressed as $\left(1+10^{0.4 \Delta m}\right)^{-1}$, and $a$ is the semimajor axis of the relative orbit of the two components. From this relation, it is evident that equal-mass/equal-flux binaries would show no perturbation, i.e., the photocenter does not move. Therefore, a star showing a perturbation has a companion that is unequal in both flux and mass ${ }^{20}$, but due to the degeneracy between the

\footnotetext{
${ }^{20}$ One exception is the rare case of a red dwarf/white dwarf pair in which the two stars may have equal fluxes or equal masses, but not both at a given wavelength.
}

scaling of the photocentric and relative orbits and mass ratio/ flux difference, there is uncertainty in the nature of the companion. However, inferences can be made using the position of a star with an unseen companion on the HR diagram, such as shown in Figure 4 (see Section 6 below for further discussion). For example, a star elevated in the HR diagram has a companion that contributes significant flux, whereas a star that is not elevated has a lower luminosity secondary-for these red dwarfs, perhaps a white dwarf or brown dwarf.

The nightly mean astrometric residual plots in R.A. and decl. for the 16 stars exhibiting perturbations are shown in Figure 8. A star without a perturbation would have residuals very close to the " 0 " line, such as shown in the upper left panel of Figure 8 for SCR 1656-2046. The magnitudes of most perturbations are typically less than $\sim 20$ mas, but several stars show much larger perturbations-SCR 0723-8015, LP 848-050AB, L 408$123 \mathrm{AB}$, LTT 7434AB, and LTT 9828AB. Note that the $y$ axis scales for these five systems extend to 60 mas or 100 mas, rather than the standard 40 mas plotted for the other 12 stars. We note that there are gaps in the temporal coverage for some 
Table 3

Orbital Parameters

\begin{tabular}{|c|c|c|c|c|c|c|c|c|}
\hline $\begin{array}{l}\text { Name } \\
\text { (1) }\end{array}$ & $\begin{array}{l}\text { Filt } \\
\text { (2) }\end{array}$ & $\begin{array}{c}P \\
\text { (year) } \\
(3)\end{array}$ & $\begin{array}{c}a \\
(\mathrm{mas}) \\
(4)\end{array}$ & $\begin{array}{l}e \\
(5)\end{array}$ & $\begin{array}{c}i \\
(\mathrm{deg}) \\
(6)\end{array}$ & $\begin{array}{l}\text { Long. Nodes }(\Omega) \\
\text { (deg) } \\
\text { (7) }\end{array}$ & $\begin{array}{l}\text { Long. Periastron }(\omega) \\
(\mathrm{deg}) \\
(8)\end{array}$ & $\begin{array}{c}T \\
\text { (year) } \\
(9)\end{array}$ \\
\hline SCR $1230-3411 \mathrm{AB}$ & $R$ & $4.68 \pm 0.08$ & $20.1 \pm 2.1$ & $0.57 \pm 0.17$ & $86.2 \pm 2.9$ & $234.8 \pm 5.3$ & $77.4 \pm 3.6$ & $2011.36 \pm 0.06$ \\
\hline LEHPM 1-4771AB & $R$ & $5.75 \pm 0.13$ & $20.2 \pm 1.5$ & $0.04 \pm 0.06$ & $106.5 \pm 3.8$ & $267.7 \pm 4.7$ & $277.4 \pm 42.1$ & $2012.26 \pm 0.68$ \\
\hline LTT 9828AB & V & $11.39 \pm 0.18$ & $33.4 \pm 1.8$ & $0.32 \pm 0.03$ & $31.7 \pm 7.7$ & $133.6 \pm 14.4$ & $96.9 \pm 14.1$ & $2001.74 \pm 0.20$ \\
\hline
\end{tabular}

stars, as they were removed but then re-added to the astrometry program after a period of a few years. Systems within $25 \mathrm{pc}$ will continue to be monitored to complete orbital coverage and improve orbital elements.

\subsection{New Multiples-Orbital Fits for Four Systems}

For four of the unresolved systems, we see clear perturbations in both the R.A. and decl. axes for which the astrometry coverage spans what appear to be full orbits. ${ }^{21}$ (For the other 12 systems exhibiting perturbations, the orbits have not yet wrapped due to insufficient time coverage; thus, we do not provide the orbital elements here, as they are not useful in isolation.

We use the methodology described in Hartkopf et al. (1989) that uses a least-squares technique to solve for the orbital elements, given initial guesses of three elements: orbital period $(P)$, epoch of periastron $(T)$, and eccentricity $(e)$. Sets of initial guesses are typically used to explore possible combinations for orbital parameters to find the true minimum for the fit, rather than a local minimum. The orbital fits for the four systems are shown in Figure 9 and the elements for the photocentric orbits are given in Table 3. The periods and semimajor axes are generally reliable, while the other orbital elements are less welldetermined. We also include the filter in which the observations were made because the brightness ratios between components and the consequent photocenter perturbations will vary with wavelength.

\subsection{Multiplicity Fraction}

In addition to the 16 stars with unseen companions described in Section 5.1, there are 16 target stars with previously known companions, or new companions revealed directly for the first time in our images (e.g., LTT 4004B, LHS 5348B, etc.). All 32 of the systems discussed are currently known to be binaries, some of which have been presented before in this series of papers; no higher order multiples, e.g., triples, quadruples, etc., are yet known. Table 4 lists the multiplicity information for all 32 systems in this sample of 151 systems. Listed are the separations $(\rho)$ and position angles $(\theta)$ of the companions from their primaries, the dates of the measurements, the techniques used to identify the binaries, and the references for the separation measurements and the detection technique. Where available, the magnitude differences between the components ( $\Delta$ mag), the filters in which the measurements were made, and the references for the $\Delta$ mags are also listed.

\footnotetext{
21 LP 349-25AB also has a wrapped orbit in our data, but we have HST-WFC3 data that will provide a detailed picture of the system and which will be described in a future paper in this series.
}

Newly presented separations were calculated using SExtractor (Bertin \& Arnouts 1996). The delta-magnitudes $(\Delta \mathrm{mag})$ for binaries with small separations $\left(\lesssim 5^{\prime \prime}\right)$ were deblended via PSF photometry, as described in Section 3.1.1. The images used for the $\Delta$ mag calculations are usually those in which the parallax was measured; thus, the filters listed are the parallax filters for each system.

Of the 124 systems presented here that are within $25 \mathrm{pc}, 29$ are binary, resulting in a multiplicity fraction of $23 \pm 4 \%$. We consider this a lower limit, as there are likely additional multiples yet to be identified, as described in the next section. Nonetheless, the derived multiplicity fraction is in agreement with the recently determined value found in the much larger sample of the first author (Winters 2015), in which a value of $27.4 \pm 1.3 \%$ was measured for a large sample of 1122 carefully vetted $\mathrm{M}$ dwarfs known to be within $25 \mathrm{pc}$.

\subsection{Candidate Multiples or Young Stars?}

As illustrated in Figure 3, there are 12 stars with trigonometric distance measurements that are at least sqrt(2) times larger than the estimated photometric distances. These stars are coincident with those elevated above the main sequence in the observational HR diagram of Figure 4. There are two straightforward explanations for these stars- they are either unresolved multiples and/or young stars.

While we noted earlier that the sample had been vetted for young stars prior to being measured for astrometry, the new parallax measurements provide an extra parameter with which to analyze these stars for indicators of youth. One attribute of young stars is a low tangential velocity, $V_{\tan }$, because as a group, young stars have not had the many interactions with objects in the Galactic disk that lead to disk-heating, and consequently higher $V_{\tan }$ values (see Leggett 1992 for red dwarfs in particular). While we cannot pinpoint an individual young star by $V_{\tan }$ alone, we can at least examine the 12 outlier stars as a group. $V_{\tan }$ can be derived from $\pi_{\text {trig }}$ and proper motion, and is listed in Table 2 for the entire sample, and noted in Table 5 for the 12 stars that are overluminous. As can be seen in the histogram of Figure 10, the $V_{\tan }$ values for the 12 candidates are not unusually low, which would be expected for a sample of young stars.

Young stars are also generally coronally active, which results in stars' detections in the X-ray and ultraviolet (UV) regions of the electromagnetic spectrum. We searched for these 12 targets in the ROentgen SATellite (ROSAT) All Sky Survey X-ray catalog (Voges et al. 1999, 2000) and the Galaxy Evolution Explorer (GALEX) DR5 UV catalog (Bianchi et al. 2011). Because our targets have substantial proper motion, we used these proper motions to move the targets to their 1991 positions before searching for them in ROSAT, using a search radius of 
Table 4

Information for Confirmed Multiples

\begin{tabular}{|c|c|c|c|c|c|c|c|c|c|c|}
\hline Name & $\begin{array}{c}\text { R.A. } \\
\text { (hh:mm:ss) }\end{array}$ & $\begin{array}{c}\text { Decl. } \\
\text { (dd:mm:ss) }\end{array}$ & $\begin{array}{c}\rho \\
(")\end{array}$ & $\begin{array}{c}\theta \\
(\mathrm{deg})\end{array}$ & Year & Technique & Ref & $\begin{array}{l}\Delta \mathrm{mag} \\
(\mathrm{mag})\end{array}$ & Filter & Ref \\
\hline LP 349-25AB & 002756.0 & +221933 & $<1$ & $\ldots$ & 2015 & astdet & 1 & 0.38 & $\mathrm{H}$ & 3 \\
\hline L 294-92AB & 014742.6 & -483606 & $<1$ & $\ldots$ & 2016 & astdet & 1 & $\ldots$ & $\ldots$ & $\ldots$ \\
\hline L $225-57 \mathrm{AB}$ & 023421.2 & -530537 & $<1$ & $\ldots$ & $\ldots$ & SB2 & 2 & $\ldots$ & $\ldots$ & $\ldots$ \\
\hline LP $831-45 \mathrm{AB}$ & 031418.0 & -230931 & $<1$ & $\ldots$ & 2016 & astdet & 1 & $\ldots$ & $\ldots$ & $\ldots$ \\
\hline L 591-42AB & 043640.9 & $-2721 \quad 18$ & 49.58 & 196 & 2001 & visdet & 5 & 0.31 & $V_{J}$ & 5 \\
\hline SCR $0723-8015 A B$ & 072400.8 & -801522 & $<1$ & $\ldots$ & 2016 & astdet & 1 & $\ldots$ & $\ldots$ & $\ldots$ \\
\hline SCR $0838-5855 \mathrm{AB}$ & 083802.3 & -585557 & $<1$ & $\ldots$ & 2016 & astdet & 1 & $\ldots$ & $\ldots$ & $\ldots$ \\
\hline LP 788-1AB & 093122.3 & -171743 & $<1$ & $\ldots$ & 2016 & astdet & 1 & $\ldots$ & $\ldots$ & $\ldots$ \\
\hline L 392-39AB & 101951.0 & -414848 & 30.91 & 120 & 2001 & visdet & 5 & 1.53 & $V_{J}$ & 5 \\
\hline LP 848-50AB & 104241.3 & -241604 & $<1$ & $\ldots$ & 2016 & astdet & 1 & $\ldots$ & $\ldots$ & $\ldots$ \\
\hline LTT 4004AB & 105442.0 & -071833 & $<2$ & $\ldots$ & 2016 & visdet & 1 & $\ldots$ & $\ldots$ & $\ldots$ \\
\hline SCR $1630-3633 \mathrm{AB}$ & 163027.2 & -363356 & 2.0 & 247 & 2015 & visdet & 1 & 4.6 & $V_{J}$ & 1 \\
\hline G $139-3 \mathrm{AB}$ & 165825.0 & +135806 & 0.822 & 294 & 2008 & spkdet & 4 & 0.48 & $754 \mathrm{~nm}$ & 4 \\
\hline G $154-43 \mathrm{AB}$ & 180336.0 & -185850 & $<1$ & $\ldots$ & 2015 & astdet & 1 & $\ldots$ & $\ldots$ & \\
\hline L $43-72 \mathrm{AB}$ & 181115.3 & -785923 & $<1$ & $\ldots$ & $\ldots$. & SB2 & 2 & $\ldots$ & $\ldots$ & $\cdots$ \\
\hline LTT 7246AB & 181512.4 & -192407 & 3.5 & 219 & 2015 & visdet & 1 & 2.2 & $V_{J}$ & 1 \\
\hline LTT 7419AB & 184312.5 & -332246 & 14.90 & 354 & 2001 & visdet & 5 & 5.99 & $V_{J}$ & 1 \\
\hline LTT 7434AB & 184557.5 & -285553 & $<1$ & $\ldots$ & 2015 & astdet & 1 & $\ldots$ & $\ldots$ & $\ldots$ \\
\hline L $345-91 \mathrm{AB}$ & 184841.4 & -464708 & 2.3 & 032 & 2009 & visdet & 1 & 0.1 & $V_{J}$ & 1 \\
\hline LHS 5348AB & 192752.6 & -281115 & $<2$ & $\ldots$ & 2015 & visdet & 1 & $\ldots$ & $\ldots$ & $\ldots$ \\
\hline LEHPM 2-1265AB & 203301.9 & -490311 & $<1$ & $\ldots$ & 2015 & astdet & 1 & $\ldots$ & $\ldots$ & $\ldots$ \\
\hline L $427-34 \mathrm{AB}$ & 214911.4 & -413330 & 2.5 & 139 & 2011 & visdet & 1 & 0.3 & $V_{J}$ & 1 \\
\hline LEHPM 1-4771AB & 223009.5 & -534456 & 0.020 & $\ldots$ & 2015 & astorb & 1 & $\ldots$ & $\ldots$ & $\ldots$ \\
\hline L 645-74AB & 223823.0 & -292100 & 14.57 & 136 & 2002 & visdet & 5 & 1.71 & $V_{J}$ & 5 \\
\hline
\end{tabular}

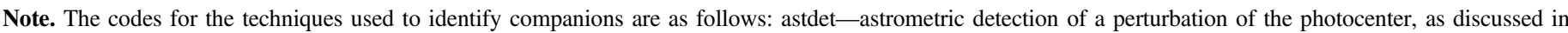

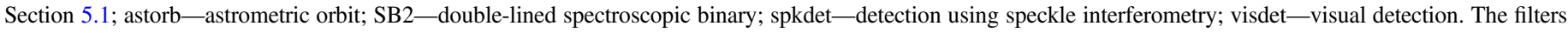

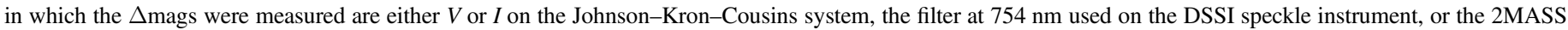
$H$-band.

References. (1) This work, (2) Bonfils et al. (2013), (3) Forveille et al. (2005), (4) Horch et al. (2010), (5) Jao et al. (2003), (6) Winters et al. (2011).

25", as per Voges et al. (1999). The midpoint of the GALEX survey was 2007 , so we used the stars' proper motions to find their 2007 positions before performing the search, using a search radius of $5^{\prime \prime}$.

Five of the 12 overluminous stars were found to have detections in the ROSAT and GALEX catalogs, identifying them as coronally active, and therefore likely young objects. One star LEHPM 2-3528 was detected in ROSAT, while four stars were detected in the GALEX catalog: LTT 313, SCR 0211-3504, SCR 2025-1534, and LP 567-63. LTT 313 had both far-UV and near-UV (NUV) detections, while the other four were detected in the NUV only.

We also checked the space motions of the stars, to see if they kinematically match any of the known nearby young moving groups. For this, we used the LocAting Constituent mEmbers In Nearby Groups (LACEwING) moving group identification code (A. Riedel et al. 2016, in preparation). LACEwING uses positions, proper motions, parallaxes, and radial velocities to identify objects that might share the space motions and positions of 13 nearby young moving groups and three open clusters within $100 \mathrm{pc}$ of the Sun. It has two modes, a field star mode in which it assumes nothing is known about the age of the star, and a young star mode, where it is assumed that the object is young. As these 12 stars are overluminous, we used the young star mode for all of them. Although we do not have radial velocities for these stars, using our positions, proper motions, and parallaxes, we identify SCR 0027-0806 as a lowprobability $(28 \%)$ member of the $\sim 45 \mathrm{Myr}$ old (Kraus et al. 2014; Bell et al. 2015) Tucana-Horologium moving group; SCR 0724-3125 low-probability member of the $\sim 200 \mathrm{Myr}$ old Carina-Near moving group (Zuckerman et al. 2006), with a probability of 28\%; SCR 1901-3106 and SCR 2025-1534 as low- and moderate-probability members of the $130-150$ Myr old AB Doradus moving group (Luhman et al. 2005; Bell et al. 2015) (probabilities of $27 \%$ and 57\%, respectively); and LP 567-63 as a member of the $\sim 50$ Myr old Argus moving group (Torres et al. 2008), with a probability of $38 \%$. We note that both SCR 2025-1534 and LP 567-63 were found to have detections in GALEX.

Based on these analyses, we suspect that half of these highlighted systems are unresolved multiples, with the other half possibly being young. 
Table 5

Candidate Multiples and Young Stars

\begin{tabular}{|c|c|c|c|c|}
\hline Name & $\begin{array}{c}\text { R.A. } \\
\text { (hh:mm:ss) }\end{array}$ & $\begin{array}{c}\text { Decl. } \\
\text { (dd:mm:ss) }\end{array}$ & $\begin{array}{c}V_{\tan } \\
\left(\mathrm{km} \mathrm{s}^{-1}\right)\end{array}$ & $\begin{array}{l}\text { Youth } \\
\text { Indicator }\end{array}$ \\
\hline SCR 0027-0806 & 002745.4 & -080605 & 22.6 & Tuc-Hor \\
\hline LTT 313 & 003538.1 & -100419 & 30.1 & $G A L E X$ \\
\hline L 2-60 & 012920.8 & -855611 & 63.4 & $\ldots$ \\
\hline SCR 0211-0354 & 021151.7 & -035403 & 23.7 & $G A L E X$ \\
\hline LEHPM 2-3528 & 060758.1 & $\begin{array}{lll}-61 & 15 & 11\end{array}$ & 42.1 & ROSAT \\
\hline SCR 0724-3125 & 072421.2 & -312558 & 24.7 & Carina-Near \\
\hline SCR 0733-4406 & 073342.7 & -440613 & 57.4 & $\ldots$ \\
\hline L 532-12 & 085402.4 & -305137 & 39.2 & $\cdots$ \\
\hline LHS 5231 & 125918.2 & -001033 & 64.8 & $\ldots$ \\
\hline SCR 1901-3106 & 190159.2 & -310645 & 43.0 & AB Dor \\
\hline SCR 2025-1534 & 202508.6 & -153416 & 29.9 & AB Dor, GALEX \\
\hline LP 567-63 & 203431.1 & -323100 & 26.1 & Argus, GALEX \\
\hline
\end{tabular}

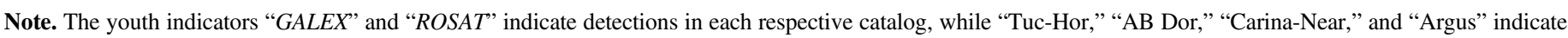
the young nearby moving groups to which the star in question has a probability of belonging, based on its kinematic information.

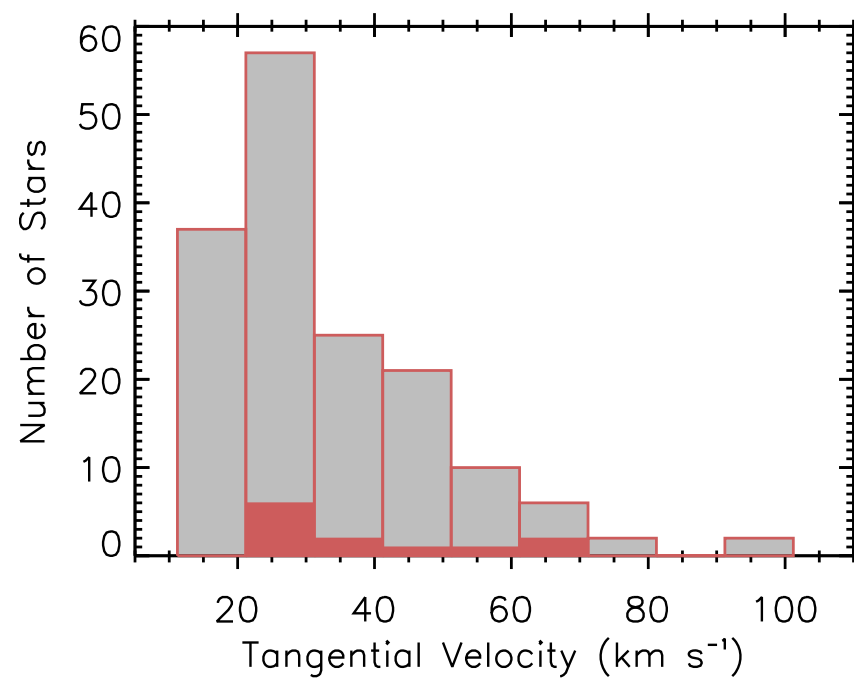

Figure 10. Distribution of the tangential velocities of the the stars presented here. The 12 stars identified as candidate binaries are shown in pink.

\section{DISCUSSION: THE NEARBY M DWARF POPULATION}

Recent noteworthy surveys have considerably improved our knowledge of the northern M dwarf population, with Dittmann et al. (2014) contributing 1507 and Finch \& Zacharias (2016) adding $1059 \pi_{\text {trig }}$ measurements for our nearby low-mass neighbors. Yet, the southern sky has remained largely untouched. RECONS' work is improving the census of $\mathbf{M}$ dwarfs with accurate distances in the southern hemisphere.

Prior to this paper, there were 448 southern M dwarf systems with accurate (uncertainties less than 10 mas) published $\pi_{\text {trig }}$ placing them within $25 \mathrm{pc}$. This number includes measurements from YPC (192), HIP (88), previous publications in this series by RECONS (131), and a few dozen systems from other efforts. The trigonometric distances for the 116 systems within $25 \mathrm{pc}$ and south of decl. $=0$ presented here increase the census of $\mathrm{M}$ dwarf systems in the southern sky by $26 \%$. The population density diagram for the southern sky shown in Figure 11, in which we use use $M_{V}$ as a proxy for mass, provides a gauge of the uniformity of systems within a volume

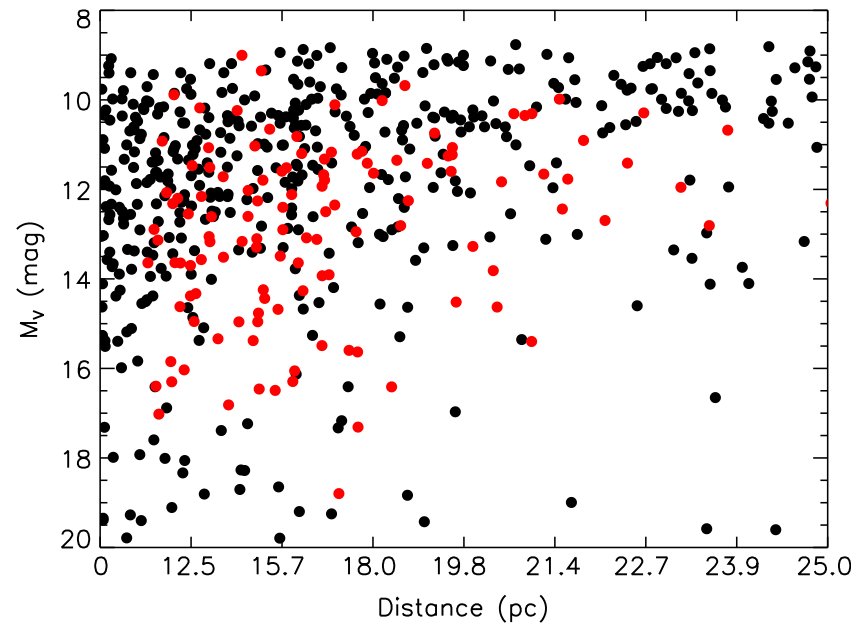

Figure 11. Population density diagram of southern $M$ dwarfs within $25 \mathrm{pc}$, with distance to $25 \mathrm{pc}$ indicated in intervals corresponding to eight equalvolume shells. Illustrated in red are the 123 new nearby (distances $<25 \mathrm{pc}$ ) M dwarfs (in 116 systems) with accurate $\pi_{\text {trig }}$ from this work.

stretching to $25 \mathrm{pc}$, broken into eight equal volume shells, with the first shell incorporating half of the distance, but one-eighth of the volume, to the horizon. The 116 new southern members added by this work are indicated in red. Intrinsically faint, latetype $\mathrm{M}$ dwarfs are more prominent at closer distances than their mid-type counterparts at further distances. ${ }^{22}$ The paucity of late M dwarfs at larger distances is clearly an observational bias, as their faintness has precluded their identification and parallax measurement to date.

\section{FUTURE}

Members of the RECONS group are stellar cartographers, driven to discover and characterize the nearby population of stars. While previous work has reported the census of the $5 \mathrm{pc}$ sample (Henry 2015, pp. 286-290) and 10 pc sample (Henry et al. 2006 and T. J. Henry 2016, in preparation), we are

\footnotetext{
$\overline{22}$ All systems reported here are beyond $10 \mathrm{pc}$, as the closer systems will be described in an upcoming paper in this series.
} 
expanding our investigations of the nearest stars to $25 \mathrm{pc}$. This $25 \mathrm{pc}$ horizon limit has been adopted as a match to the canonical distance limit of the Third Catalog of Nearby Stars (Gliese \& Jahreiß, 1991) and ultimately should include 5000 stellar systems.

Development by the RECONS group of a modern catalog listing all stars, brown dwarfs, and planets located within $25 \mathrm{pc}$, with distances determined only via $\pi_{\text {trig }}$, has been underway for the past few years. In this compendium, called the RECONS 25 Parsec Database, is a wealth of information on each system, with only data from carefully vetted publications selected for inclusion. The current version, as of 2016 August 01, includes 2826 systems containing 3801 stars, brown dwarfs, and planets, and we anticipate releasing it to the astronomical community in the near future. The main criterion for inclusion is that each system must have a published trigonometric parallax of at least 40 mas, with an uncertainty $\leqslant 10$ mas; thus, 124 of the systems reported here will be new entries to the database.

The long-anticipated results from Gaia will measure distances for many nearby stars currently lacking accurate $\pi_{\text {trig }}$ measurements. But, ground-based astrometry will continue to be able to push to the end of the stellar main sequence at $M_{V}=21.5$ (Dieterich et al. 2014) all the way to $25 \mathrm{pc}$, a realm unlikely to be reached by Gaia. Thus, there will continue to be a need for ground-based astrometry efforts. As the RECONS observing program began at the $0.9 \mathrm{~m}$ as an NOAO Survey in 1999, the data series now stretches to over a decade for hundreds of nearby southern $\mathbf{M}$ dwarfs, providing long-term astrometry and photometry coverage that will likely not be available from Gaia, nor any other project for the foreseeable future. Thus, this unique temporal data set coverage is valuable for studies of these low-mass neighbors, especially those exhibiting perturbations for which mass determinations will be possible. In addition, RECONS' long-term monitoring of these potential exoplanet host stars enables investigations of their long-term photometric trends, such as variability and stellar cycles, that may affect life on any planets around these lowmass neighbors.

This research was made possible by NSF grants AST 0507711, AST 09-08402, and AST 14-12026. We also thank the members of the SMARTS Consortium, who have enabled the operations of the small telescopes at CTIO since 2003, as well as the observer support at CTIO, specifically Edgardo Cosgrove, Arturo Gomez, Manuel Hernandez, Alberto Miranda, Mauricio Rojas, Hernan Tirado, and Joselino Vasquez. We thank the referee for a very thorough review of the paper that has allowed us to improve it.

This research has made use of data obtained from the SuperCOSMOS Science Archive, prepared and hosted by the Wide Field Astronomy Unit, Institute for Astronomy, University of Edinburgh, which is funded by the UK Science and Technology Facilities Council. Data products from the Two Micron All Sky Survey, which is a joint project of the University of Massachusetts and the Infrared Processing and Analysis Center/California Institute of Technology, funded by the National Aeronautics and Space Administration and the National Science Foundation have been used extensively, as have the SIMBAD database and the Aladin and Vizier interfaces, operated at CDS, Strasbourg, France. Data from the ROSAT and GALEX NASA missions were used in this publication.

J.G.W. is currently supported by a grant from the John Templeton Foundation. The opinions expressed here are those of the authors and do not necessarily reflect the views of the John Templeton Foundation.

\section{REFERENCES}

Bartlett, J. L., Ianna, P. A., \& Begam, M. C. 2009, PASP, 121, 365 Bell, C. P. M., Mamajek, E. E., \& Naylor, T. 2015, MNRAS, 454, 593 Benedict, G. F., Henry, T. J., Franz, O. G., et al. 2016, AJ, 152, 141 Bertin, E., \& Arnouts, S. 1996, A\&AS, 117, 393

Bessel, M. S. 1990, A\&AS, 83, 357

Bianchi, L., Herald, J., Efremova, B., et al. 2011, AP\&SS, 335, 161 Bonfils, X., Delfosse, X., Udry, S., et al. 2013, A\&A, 549, A109

Costa, E., Méndez, R. A., Jao, W.-C., et al. 2005, AJ, 130, 337

Costa, E., Méndez, R. A., Jao, W.-C., et al. 2006, AJ, 132, 1234

Dahn, C. C., Harris, H. C., Vrba, F. J., et al. 2002, AJ, 124, 1170

Davison, C. L., White, R. J., Henry, T. J., et al. 2015, AJ, 149, 106

Dieterich, S. B., Henry, T. J., Jao, W.-C., et al. 2014, AJ, 147, 94

Dittmann, J. A., Irwin, J. M., Charbonneau, D., \& Berta-Thompson, Z. K. 2014, ApJ, 784, 156

Dupuy, T. J., \& Liu, M. C. 2012, ApJS, 201, 19

Fabricius, C., \& Makarov, V. V. 2000, A\&AS, 144, 45

Finch, C. T., \& Zacharias, N. 2016, AJ, 151, 160

Forveille, T., Beuzit, J.-L., Delorme, P., et al. 2005, A\&A, 435, L5

Gatewood, G. 2008, AJ, 136, 452

Gatewood, G., \& Coban, L. 2009, AJ, 137, 402

Gizis, J. E., Jao, W.-C., Subasavage, J. P., \& Henry, T. J. 2007, ApJL, 669, L45

Gliese, W., \& Jahreiß, H. 1991, Preliminary Version of the Third Catalogue of Nearby Stars, Tech. Rep.

Graham, J. A. 1982, PASP, 94, 244

Gray, R. O., Corbally, C. J., Garrison, R. F., McFadden, M. T., \& Robinson, P. E. 2003, AJ, 126, 2048

Hambly, N. C., Henry, T. J., Subasavage, J. P., Brown, M. A., \& Jao, W.-C. 2004, AJ, 128, 437

Hartkopf, W. I., McAlister, H. A., \& Franz, O. G. 1989, AJ, 98, 1014

Hawley, S. L., Gizis, J. E., \& Reid, I. N. 1996, AJ, 112, 2799

Henry, T. J. 2015, in The Observer's Handbook, ed. D. M. F. Chapman (Toronto, Ontario: The Royal Astronomical Society of Canada), 286

Henry, T. J., Ianna, P. A., Kirkpatrick, J. D., \& Jahreiss, H. 1997, AJ, 114, 388

Henry, T. J., Jao, W.-C., Subasavage, J. P., et al. 2006, AJ, 132, 2360

Henry, T. J., Subasavage, J. P., Brown, M. A., et al. 2004, AJ, 128, 2460

Horch, E. P., Falta, D., Anderson, L. M., et al. 2010, AJ, 139, 205

Hosey, A. D., Henry, T. J., Jao, W.-C., et al. 2015, AJ, 150, 6

Ianna, P. A. 1993, in IAU Symp. 156, Developments in Astrometry and their Impact on Astrophysics and Geodynamics, ed. I. I. Mueller \& B. Kolaczek (Kluwer Academic Publishers), 75

Ianna, P. A., \& Bessell, M. S. 1986, PASP, 98, 658

Jao, W.-C., Henry, T. J., Subasavage, J. P., et al. 2003, AJ, 125, 332

Jao, W.-C., Henry, T. J., Subasavage, J. P., et al. 2005, AJ, 129, 1954

Jao, W.-C., Henry, T. J., Subasavage, J. P., et al. 2011, AJ, 141, 117

Jao, W.-C., Henry, T. J., Subasavage, J. P., et al. 2014, AJ, 147, 21

Jefferys, W. H., Fitzpatrick, M. J., \& McArthur, B. E. 1988, CeMec, 41, 39

Kraus, A. L., Shkolnik, E. L., Allers, K. N., \& Liu, M. C. 2014, AJ, 147, 146 Landolt, A. U. 1992, AJ, 104, 372

Landolt, A. U. 2007, AJ, 133, 2502

Landolt, A. U. 2013, AJ, 146, 131

Leggett, S. K. 1992, ApJS, 82, 351

Lèpine, S., Thorstensen, J. R., Shara, M. M., \& Rich, R. M. 2009, AJ, 137, 4109

Luhman, K. L., Stauffer, J. R., \& Mamajek, E. E. 2005, ApJL, 628, L69

Lurie, J. C., Henry, T. J., Jao, W.-C., et al. 2014, AJ, 148, 91

Luyten, W. J. 1979a, LHS Catalogue (2nd ed.; Minneapolis, MN: Univ. Minnesota Press)

Luyten, W. J. 1979b, NLTT Catalogue, Vol._I. +90_to_+30_. Vol._II. +30_to_0_(Minneapolis, MN: Univ. Minnesota Press)

Luyten, W. J. 1980a, NLTT Catalogue, Vol._III. 0_to -30_ (Minneapolis, MN: Univ. Minnesota Press)

Luyten, W. J. 1980b, NLTT Catalogue, Vol._IV. -30_to_-90_ (Minneapolis, MN: Univ. Minnesota Press)

Mamajek, E. E., Bartlett, J. L., Seifahrt, A., et al. 2013, AJ, 146, 154

Perryman, M. A. C., Lindegren, L., Kovalevsky, J., et al. 1997, A\&A, 323, L49

Reid, I. N., Hawley, S. L., \& Gizis, J. E. 1995, AJ, 110, 1838

Riedel, A. R., Cutri, R. M., Stiening, R., et al. 2014, AJ, 147, 85 
Riedel, A. R., Murphy, S. J., Henry, T. J., et al. 2011, AJ, 142, 104

Riedel, A. R., Subasavage, J. P., Finch, C. T., et al. 2010, AJ, 140, 897

Schlesinger, F. 1911, ApJ, 33, 8

Shkolnik, E. L., Anglada-Escudé, G., Liu, M. C., et al. 2012, ApJ, 758, 56

Skrutskie, M. F., Cutri, R. M., Stiening, R., et al. 2006, AJ, 131, 1163

Smart, R. L., Ioannidis, G., Jones, H. R. A., Bucciarelli, B., \& Lattanzi, M. G. 2010, A\&A, 514, A84

Smart, R. L., Lattanzi, M. G., Jahreiß, H., Bucciarelli, B., \& Massone, G. 2007, A\&A, 464, 787

Söderhjelm, S. 1999, A\&A, 341, 121

Subasavage, J. P., Jao, W.-C., Henry, T. J., et al. 2009, AJ, 137, 4547

Tody, D. 1993, in ASP Conf. Ser. 52, Astronomical Data Analysis Software and Systems II, ed. R. J. Hanisch, R. J. V. Brissenden, \& J. Barnes (San Francisco, CA: ASP), 173
Torres, C. A. O., Quast, G. R., Melo, C. H. F., \& Sterzik, M. F. 2008, in Young Nearby Loose Associations, ed. B. Reipurth (The Southern Sky ASP Monograph Publications), 757

van Altena, W. F., Lee, J. T., \& Hoffleit, D. 1995, yCat, 1174, 0

van de Kamp, P. 1975, ARA\&A, 13, 295

van Leeuwen, F. 2007, Hipparcos, The New Reduction of the Raw Data, Vol. 350 (Springer), 20

Voges, W., Aschenbach, B., Boller, T., et al. 1999, A\&A, 349, 389

Voges, W., Aschenbach, B., Boller, T., et al. 2000, IAUC, 7432, 1

von Braun, K., Boyajian, T. S., Kane, S. R., et al. 2011, ApJL, 729, L26

Winters, J. G. 2015, PhD thesis, Georgia State Univ.

Winters, J. G., Henry, T. J., Jao, W.-C., et al. 2011, AJ, 141, 21

Winters, J. G., Henry, T. J., Lurie, J. C., et al. 2015, AJ, 149, 5

Zuckerman, B., Bessell, M. S., Song, I., \& Kim, S. 2006, ApJL, 649, L115 